\title{
MEASUREMENT OF THE FORM FACTOR RATIOS IN SEMILEPTONIC DECAYS OF CHARM MESONS
}

\author{
A DISSERTATION \\ SUBMITTED TO THE DEPARTMENT OF PHYSICS \\ AND THE COMMITTEE ON GRADUATE STUDIES \\ OF STANFORD UNIVERSITY \\ IN PARTIAL FULFILLMENT OF THE REQUIREMENTS \\ FOR THE DEGREE OF \\ DOCTOR OF PHILOSOPHY
}

By

Renata Zaliznyak

May 1998 
(c) Copyright 1998 by Renata Zaliznyak All Rights Reserved 
I certify that I have read this dissertation and that in my opinion it is fully adequate, in scope and quality, as a dissertation for the degree of Doctor of Philosophy.

Patricia Burchat

(Principal Advisor)

I certify that I have read this dissertation and that in my opinion it is fully adequate, in scope and quality, as a dissertation for the degree of Doctor of Philosophy.

\section{Giorgio Gratta}

I certify that I have read this dissertation and that in my opinion it is fully adequate, in scope and quality, as a dissertation for the degree of Doctor of Philosophy.

\section{Jeffrey Willick}

Approved for the University Committee on Graduate Studies: 


\section{Abstract}

I have measured the form factor ratios $r_{2}=A_{2}(0) / A_{1}(0)$ and $r_{V}=V(0) / A_{1}(0)$ in the semileptonic charm meson decay $D^{+} \rightarrow \bar{K}^{* 0} e^{+} \nu_{e}$ from data collected by the Fermilab E791 collaboration.

Form factors are introduced in the calculation of the hadronic current in semileptonic decays of strange, charm, or bottom mesons, such as $D^{+} \rightarrow \bar{K}^{* 0} e^{+} \nu_{e}$. Semileptonic decays provide insight into quark coupling to the $W$ boson since the leptonic and hadronic amplitudes in the Feynman diagram for the decay are completely separate. There are no strong interactions between the final state leptons and quarks. A number of theoretical models predict the values of the form factors for $D^{+} \rightarrow \bar{K}^{* 0} e^{+} \nu_{e}$, though there is a large range of predictions.

E791 is a hadroproduction experiment that recorded over 20 billion interactions with a $500 \mathrm{GeV} \pi^{-}$beam incident on five thin targets during the 1991-92 Fermilab fixed-target run. Approximately $3000 D^{+} \rightarrow \bar{K}^{* 0} e^{+} \nu_{e}$ decays are fully reconstructed. In order to extract the form factor ratios from the data, I implement a multidimensional unbinned maximum likelihood fit with a large sample of simulated (Monte Carlo) $D^{+} \rightarrow \bar{K}^{* 0} e^{+} \nu_{e}$ events. The large E791 data sample provides the most precise measurement of the form factor ratios to date. The measured values for the form factor ratios are $r_{2}=0.71 \pm 0.08 \pm 0.09$ and $r_{V}=1.84 \pm 0.11 \pm 0.08$. These results are in good agreement with some Lattice Gauge calculations. However the agreement with quark model predictions is not as good. 


\section{Acknowledgments}

I would like to offer my sincere gratitude to the following people, without whose assistance and support this analysis and/or my graduate school experience would not have been as successful...

To Pat Burchat, my advisor, a friend, a role model, a mentor, and an inspiration. Thank you for teaching me everything I know about Physics, and much of what I know about life. Thank you for your patience and support.

To my E791 collaborators, especially Pauline Gagnon, whose thesis work [1] launched this study, Kathy O'Shaughnessy who sustained it, and Daniel Mihalcea, whose cooperation on this analysis was invaluable. In addition, I would like to thank Jean Slaughter, Jeff Appel, Mike Sokoloff, and Milind Purohit for their advice and assistance, as well as the Kansas State University group for providing the use of their computer facilities. To colleagues and staff at UCSC and Stanford, in particular Guy Blaylock, David Kirkby, and Marcia Keating.

To Tara VanDerveer, Vanessa Nygaard, Kate Starbird, Kristin Folkl, and the rest of the Stanford women's basketball team for bringing me great happiness in my time here. Go Cardinal!

To Sasha Pursley, for holding my hand when things got rough, for her friendship and affection. To my dearest friends, Viet Nguyen and Diana Z. Sutherland, for being with me through the years, for their unwavering support and love. To Leslie Gordon, Gur Hoshen, and Jason Zien for their generosity and excellent dinners.

And finally, and most importantly, to my parents, Mark and Sofia Zaliznyak for bringing me to this country, for all they have sacrificed so that I could have all the opportunity to succeed, for their unwavering love and belief in me. 


\section{Contents}

Abstract iv

Acknowledgments $\quad$ v

1 Theoretical Motivation 1

1.1 Form Factors in the Standard Model . . . . . . . . . . . . 1

$1.2 q^{2}$ Dependence . . . . . . . . . . . . . . . 3

1.3 Differential Decay Rate . . . . . . . . . . . . . . . 4

1.4 Heavy Quark Effective Theory . . . . . . . . . . . . . 6

1.4.1 Form Factors in Bottom Decays . . . . . . . . . . . 9

1.5 Theoretical Predictions . . . . . . . . . . . . . . 10

2 E791 Experiment $\quad 13$

2.1 Overview . . . . . . . . . . . . . . . . 13

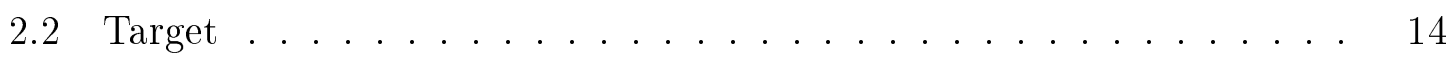

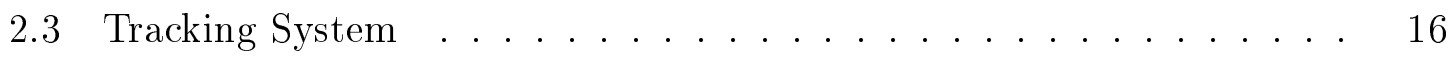

2.3.1 Silicon Microstrip Detectors . . . . . . . . . . . . 16

2.3.2 Proportional Wire Chambers _. . . . . . . . . . 17

2.3 .3 Drift Chambers . . . . . . . . . . . . . . . . . . 19

2.3 .4 Magnets . . . . . . . . . . . . . . . 20

2.4 Čerenkov Counters . . . . . . . . . . . . . . . 21

2.5 Electromagnetic Calorimeter . . . . . . . . . . . . . 24

2.6 Data Acquisition $\ldots \ldots \ldots \ldots \ldots \ldots \ldots \ldots \ldots$ 
3 Data Selection $\quad 29$

3.1 Filter and Strip . . . . . . . . . . . . . . . . 29

3.2 Substrip and Microstrip .................. 30

3.3 Nanostrip . . . . . . . . . . . . . . . . . . 31

3.4 Analysis Cut Selection . . . . . . . . . . . . . 32

$3.5 \quad D^{+} \rightarrow \bar{K}^{* 0} e^{+} \nu_{e}$ Signal . . . . . . . . . . . . . . . 35

4 Fitting Technique $\quad 39$

4.1 Maximum Likelihood Method . . . . . . . . . . . . . 39

4.2 Acceptance and Reconstruction Effects . . . . . . . . . . . . 40

4.3 Background .......................... 41

4.4 Neutrino Momentum . . . . . . . . . . . . . . . 42

5 Monte Carlo Simulation $\quad 44$

6 Particle Identification Efficiency $\quad 52$

6.1 Electron Identification . . . . . . . . . . . . . . . 52

6.2 Hadron Identification . . . . . . . . . . . . . . . . 53

$\begin{array}{lll}7 & \text { Systematic Checks } & 59\end{array}$

7.1 Fit with Known Form Factors . . . . . . . . . . . . . . . 59

7.2 Checks of Data Selection . . . . . . . . . . . . . 62

7.2.1 Final Cut Selection . . . . . . . . . . . . . 62

7.2.2 Fitting Subsamples of the Data ............ 65

7.2 .3 Four-Target Data ................... 65

7.2.4 Charge Dependence ................ 66

7.2.5 Choice of Neutrino Momentum _............ 66

7.3 Checks of Fitting Technique . . . . . . . . . . . 67

7.3.1 Number of Monte Carlo Points Associated with Data . . . . . 67

7.3.2 Monte Carlo Volume Size . . . . . . . . . . . . . . 67

7.3.3 Background Volume Size . . . . . . . . . . . . 72

7.3.4 Number of Background Events . . . . . . . . . . 72 
7.3.5 Particle Identification Efficiency Correction . . . . . . . . . . 74

7.4 Systematic Error Estimate . . . . . . . . . . . . . 76

8 Results and Discussion $\quad 77$

8.1 The Form-Factor Ratios . . . . . . . . . . . . . 77

8.2 Three-Parameter Fit . . . . . . . . . . . . . 85

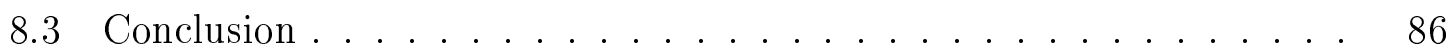

$\begin{array}{ll}\text { A Kinematic Variable Distributions } & 87\end{array}$

A.1 $\cos \theta_{V}$ v. $q^{2} \ldots \ldots \ldots \ldots \ldots$. . . . . . . . . . . . 88

A.2 $\cos \theta_{l}$ v. $q^{2} \ldots \ldots \ldots \ldots \ldots$

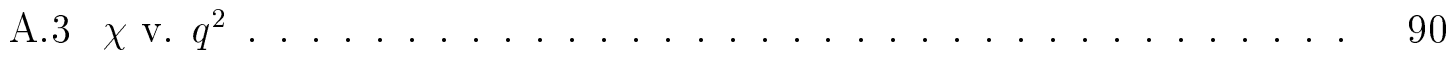

A.4 $\cos \theta_{l}$ v. $\cos \theta_{V} \ldots \ldots \ldots \ldots . \ldots \ldots$

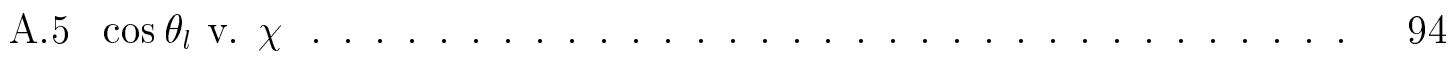

A.6 $\cos \theta_{V}$ v. $\chi \ldots \ldots \ldots \ldots \ldots \ldots$

$\begin{array}{lll}\text { B } & p_{T} \text { Discrepancy } & 96\end{array}$

$\begin{array}{ll}\text { Bibliography } & 101\end{array}$ 


\section{List of Tables}

1.1 Accessible $q^{2}$ and $w$ ranges in $D$ and $B$ decays. $\ldots \ldots \ldots \ldots$

1.2 Theoretical predictions and experimental average for the form factor ratios $r_{2}$ and $r_{V}$. The experimental average does not include the results of this analysis. . . . . . . . . . . . . . .

1.3 Theoretical predictions for the form factors $A_{1}, A_{2}$, and $V$. The experimental measurements for the form factors are done at $q^{2}=0$ and extrapolated to $q_{\max }^{2}$ using the pole form of the $q^{2}$ dependence given by Eq. 1.5. Experimental average does not include the results of this analysis. . . . . . . . . . . . . . . .

2.1 E791 target assembly. . . . . . . . . . . . . . . . . . . . .

2.2 Momentum ranges for which the Čerenkov counters can discriminate between different types of particles. . . . . . . . . . . . . . 22

2.3 The a priori probability of each particle type in a typical E791 event

5.1 Number of Monte Carlo events passing each stage of cuts. . . . . . 46

6.1 Weights used to correct the inaccuracies in the Monte Carlo electron identification efficiency for different momentum regions. EMPROB cut

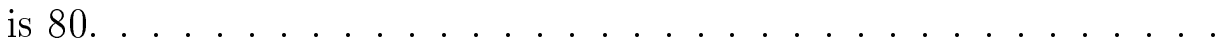

6.2 Weights correcting the inaccuracies in the Monte Carlo $K$ and $\pi$ identification efficiency for different ranges of track momentum. For both particles the probability cut is $0.4 \ldots \ldots \ldots \ldots \ldots$ 
7.1 Results for the form factor ratios from 15 different sets of CART cuts. Cut 4 is chosen as "standard". "Straight" cut refers to the set of cuts used in a previous version of this analysis. (Refer to the text for more details). The values listed here have not yet been corrected for a systematic bias due to the fitting technique. . . . . . . . . .

7.2 Results of systematic checks of data selection. The values listed here have not yet been corrected for a systematic bias due to the fitting

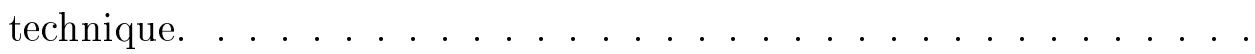

7.3 Results for the fits with both choices for the neutrino momentum. The appropriate bias due to the fitting technique is incorporated into the values for the form-factor ratios. . . . . . . . . . . . .

7.4 Results of the study of the dependence of the fit on the minimum number of Monte Carlo points required to lie in a small volume surrounding each data point. The second column lists the number of data points failing this requirement, and thus excluded from the fit. . . . . . .

7.5 Form factor ratios determined from the fit for various volume sizes for the variables $\cos \theta_{l}$ and $\cos \theta_{V} \ldots \ldots \ldots$. . . . . . . . 70

7.6 Form factor ratios determined from the fit for various volume sizes for the variables $q^{2} / q_{\text {max }}^{2}$ and $\chi \ldots \ldots \ldots 71$

7.7 Results of the checks of particle identification efficiencies. . . . . . 76

7.8 Contributions to the systematic uncertainty. . . . . . . . . . 76

8.1 Experimental measurements of $r_{2}$ and $r_{V}$ by E791 and other groups. . 78

8.2 E791 results for the form factors $A_{1}, A_{2}$, and $V$, at $q^{2}=0$ and $q^{2}=$ $q_{\text {max }}^{2}$, calculated for two different assumptions for shape of the $\bar{K}^{* 0}$ resonance. . . . . . . . . . . . . . . .

8.3 Comparison of E791 results for the form factors $A_{1}, A_{2}$, and $V$ with theoretical predictions. . . . . . . . . . . . .

8.4 The results of the fit for the parameters $r_{2}, r_{V}$, and slope, the systematic bias due to the fitting technique and the corrected values. . . . 
B.1 Weights used to correct for the discrepancy between $p_{T}$ distributions in data and Monte Carlo. The statistical uncertainty on each weight is due mostly to the error on the number of data events in each bin, and is not used to determine the form-factor ratios. . . . . . . . 98

B.2 Weights calculated from the distribution of D momentum, where the Monte Carlo events have been weighted by $p_{T}$ based weights. . . . . .

B.3 Results from three types of fits. The error is statistical. Systematic shift is not applied. . . . . . . . . . . . . 100 


\section{List of Figures}

1.1 Feynman diagram for the semileptonic decay $D^{+} \rightarrow \bar{K}^{* 0} e^{+} \nu_{e} \ldots$. . 2

1.2 Two functional forms of the $q^{2}$ dependence of the form factors for the allowed range of $q^{2}$ in the decay $D^{+} \rightarrow \bar{K}^{* 0} e^{+} \nu_{e}$ with $m_{p}=2.5 \mathrm{GeV}$. 4

1.3 Definition of the kinematic angles $\theta_{l}, \theta_{V}$, and $\chi \ldots \ldots . \ldots$

2.1 The E791 spectrometer. . . . . . . . . . . . . . 15

2.2 The primary vertex position along the beam direction. All lengths are measured in centimeters. The inset shows the fit to the third foil. Details of the fitting technique are described in the text. . . . . . 18

2.3 Diagram of a typical electromagnetic shower in the SLIC. The distances indicated on the bottom are measured in radiation lengths. Adapted from [28]. . . . . . . . . . . . . . . . . . 24

2.4 Schematic of the major components of the Data Acquisition system. . 27

3.1 Diagram of a typical E791 event of interest to this analysis. The ovals represent the resolution on the vertices. Additional tracks originating at the production vertex are not shown. . . . . . . . . . .

3.2 Right-sign (clear histogram) and wrong-sign (shaded) distribution of the linear combination chosen by CART as summarized in Eq. 3.7. Mass and particle identification cuts have already been applied. Events lying to the right of the dashed line are kept, and the rest are discarded. 36

3.3 Mass distributions for $D^{+} \rightarrow \bar{K}^{* 0} e^{+} \nu_{e}$ candidate events in the full E791 data sample. The distributions are described in the text. . . . . . 38 
5.1 Distributions of the four kinematic variables as generated by the Monte

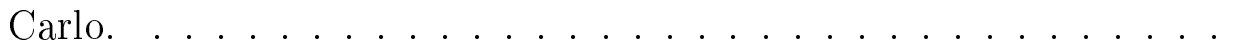

5.2 Efficiency of the reconstruction and data selection as a function of each of the four kinematic variables. The vertical scale is arbitrary. . . . .

5.3 Difference between the generated and reconstructed values of the four kinematic variables in the Monte Carlo when the negative neutrino momentum solution is used. The shaded histogram represents the smearing due solely to detector effects. All selection criteria have been applied. 49

5.4 Difference between the generated and reconstructed values of the four kinematic variables in the Monte Carlo when the positive neutrino momentum solution is used. The shaded histogram represents the smearing due solely to detector effects. All selection criteria have been applied. 50

5.5 Momentum distributions for the $D$ meson in data (crosses) and Monte Carlo (histogram). Top plot shows the magnitude of the $D$ momentum. The bottom plot shows the magnitude of the momentum component transverse to the $z$-axis. All selection criteria have been applied. . . 51

6.1 Comparison of the electron identification efficiency for EMPROB $>80$ as a function of the electron momentum for data and Monte Carlo. .

6.2 Comparison of the kaon identification efficiency (K Prob $>0.4$ ) as a function of the kaon momentum for data and Monte Carlo. Note that only kaons with momentum less than $40 \mathrm{GeV}$ are plotted in the bottom

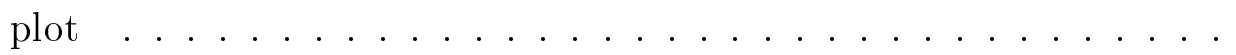

6.3 Comparison of the pion identification efficiency $(\pi$ Prob $>0.4)$ as a function of the pion momentum for data and Monte Carlo. . . . . .

7.1 Distributions of form factor ratios determined from 20 fits of Monte Carlo samples approximately the same size as the right-sign data sample. 61

7.2 Results for the form factor ratios with 15 different sets of CART cuts. The form-factor ratios used for the final result are designated with a $\operatorname{star}(\star)$. The values have not yet been corrected for a systematic bias due to the fitting technique. . . . . . . . . . . 64 
7.3 Fit estimates for the form factor ratios as the range in one kinematic variable is varied, with the other four being held constant. In the top two plots the range in $\cos \theta_{l}$ is varied; next two plots show the variation in $\cos \theta_{V}$; then $q^{2} / q_{\text {max }}^{2}$; and $\chi$ in the bottom plots. The volume size is expressed as the fraction of the total five-dimensional volume. The systematic bias due the the fitting technique is corrected for. . . . . .

7.4 Form factor ratios determined from the fit for various background volume sizes. The volume size is expressed as a fraction of the total five-dimensional volume. . . . . . . . . . . . . . . .

7.5 Form factor ratios determined from the fit for various background weights. . . . . . . . . . . . . . . .

7.6 Distributions of form factor ratios determined from the 100 fits with modified electron, kaon, and pion identification efficiency weights. Note that the horizontal scale is different the top right plot. . . . . . . .

8.1 Experimental measurements of $r_{2}$ and $r_{V}$ by E791 and other groups. For each measurement the smaller error bar is the statistical error. The larger is the statistical and systematic errors added in quadrature. . .

8.2 Background subtracted data distributions (crosses) overlaid with Monte Carlo (dashed histogram) with best fit values for the form factor ratios $\left(r_{2}=0.71, r_{V}=1.84\right)$. The $\chi^{2}$ per degree of freedom and the $\chi^{2}$ probability are shown on each plot. . . . . . . . . . . .

8.3 Background subtracted data distributions (crosses) overlaid with Monte Carlo (dashed histogram) with E653 values for the form factor ratios $\left(r_{2}=0.82, r_{V}=2.00\right)$. The $\chi^{2}$ per degree of freedom and the $\chi^{2}$ probability are shown on each plot. . . . . . . . . . . 
8.4 Background subtracted data distributions (black dots) overlaid with Monte Carlo (dashed histogram) with best fit values for the form factor ratios for (a) $\cos \theta_{V}$ for $q^{2} / q_{\max }^{2} \leq 0.5$ (top) and $q^{2} / q_{\max }^{2}>0.5$ (bottom), (b) $\cos \theta_{e}$ for $q^{2} / q_{\text {max }}^{2} \leq 0.5$ (top) and $q^{2} / q_{\text {max }}^{2}>0.5$ (bottom), (c) $\chi$ for $\cos \theta_{V} \leq 0$ (top) and $\cos \theta_{V}>0$ (bottom). The $\chi^{2}$ probability is shown on each figure. ............

A.1 The differential decay rate distribution in the space spanned by $\cos \theta_{V}$ and $q^{2}$ for four sets of form-factor ratios: a. $r_{2}=0, r_{V}=0 ;$ b. $r_{2}=1$, $r_{V}=0$; c. $r_{2}=0, r_{V}=2$; and d. $r_{2}=1, r_{V}=2 \ldots \ldots \ldots$

A.2 The differential decay rate distribution in the space spanned by $\cos \theta_{l}$ and $q^{2}$ for four sets of form-factor ratios: a. $r_{2}=0, r_{V}=0 ;$ b. $r_{2}=1$, $r_{V}=0$; c. $r_{2}=0, r_{V}=2$; and d. $r_{2}=1, r_{V}=2 \ldots \ldots . . . .$.

A.3 The differential decay rate distribution in the space spanned by $\chi$ and $q^{2}$ for four sets of form-factor ratios: a. $r_{2}=0 ., r_{V}=0 ;$ b. $r_{2}=1, r_{V}=0$; c. $r_{2}=0, r_{V}=2$; and d. $r_{2}=1, r_{V}=2 \ldots \ldots \ldots$. . . . . . . . . . . . .

A.4 The differential decay rate distribution in the space spanned by $\cos \theta_{l}$ and $\cos \theta_{V}$ for four sets of form-factor ratios: a. $r_{2}=0, r_{V}=0$; b. $r_{2}=1$, $r_{V}=0$; c. $r_{2}=0, r_{V}=2$; and d. $r_{2}=1, r_{V}=2 \ldots \ldots$. . . . .

A.5 The differential decay rate distribution in the space spanned by $\cos \theta_{l}$ and $\chi$ for four sets of form-factor ratios: a. $r_{2}=0, r_{V}=0 ;$ b. $r_{2}=1$, $r_{V}=0$; c. $r_{2}=0, r_{V}=2$; and d. $r_{2}=1, r_{V}=2 \ldots \ldots . . . .$.

A.6 The differential decay rate distribution in the space spanned by $\cos \theta_{V}$ and $\chi$ for four sets of form-factor ratios: a. $r_{2}=0, r_{V}=0 ;$ b. $r_{2}=1$, $r_{V}=0 ;$ c. $r_{2}=0, r_{V}=2$; and d. $r_{2}=1, r_{V}=2 \ldots \ldots \ldots$

B.1 Distribution of the component of D momentum transverse to the $z$-axis in data (cross hatches) and Monte Carlo (histogram) . . . . . . . . . 97

B.2 D momentum distribution in data (cross-hatches) and Monte Carlo (histograms), where the Monte Carlo are plotted with and without $p_{T}$ based weights. . . . . . . . . . . . . . . . 


\section{Chapter 1}

\section{Theoretical Motivation}

Form factors, which are defined in the following sections, are introduced in the calculation of the hadronic current in semileptonic decays of strange, charm, or bottom mesons, such as the decay of the charm meson $D^{+} \rightarrow \bar{K}^{* 0} e^{+} \nu_{e}$. This decay is represented by the Feynman diagram in Figure 1.1. Semileptonic decays provide insight into quark coupling to the $W$ boson since the leptonic and hadronic vertices in the Feynman diagram are completely separate. There are no strong interactions between the final state leptons and quarks. A number of theoretical models predict the values of the form factors, though there is a large uncertainty on these predictions. Several previous experiments have published measurements of the form factors. The large E791 data sample allows us to make the most precise measurement to date.

\subsection{Form Factors in the Standard Model}

From the Feynman diagram in Figure 1.1 we write down the amplitude for the semileptonic decay $D^{+} \rightarrow \bar{K}^{* 0} e^{+} \nu_{e}$ :

$$
A\left(D^{+} \rightarrow \bar{K}^{* 0} e^{+} \nu_{e}\right)=\frac{G_{F}}{\sqrt{2}} V_{c s} \mathcal{L}^{\mu} \mathcal{H}_{\mu}
$$

where $G_{F}$ is the Fermi coupling constant for the weak interaction, and $V_{c s}$ is the Cabibbo-Kobayashi-Maskawa (CKM) matrix element corresponding to the coupling 


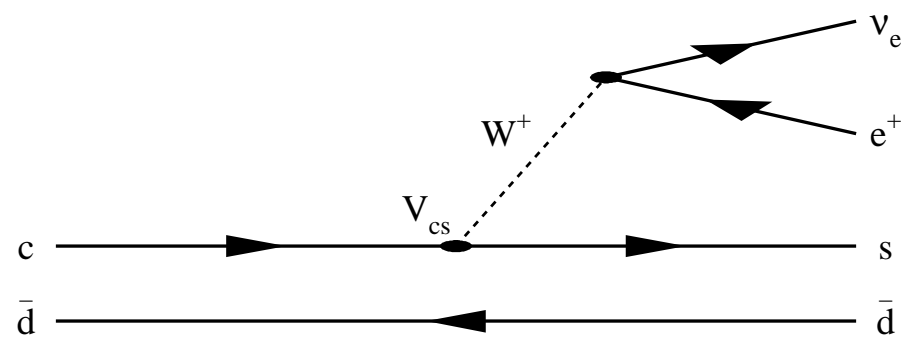

Figure 1.1: Feynman diagram for the semileptonic decay $D^{+} \rightarrow \bar{K}^{* 0} e^{+} \nu_{e}$

of the charm quark to the strange quark. In contrast to $V_{u b}$ in semileptonic decays of the $B$ meson, $V_{c s}$ is quite precisely determined from the unitarity constraints on the CKM matrix. $\mathcal{L}^{\mu}$ and $\mathcal{H}_{\mu}$ represent the leptonic and hadronic currents. The leptonic current is the standard $V-A$ interaction

$$
\mathcal{L}^{\mu}=\bar{u}_{e} \gamma^{\mu}\left(1-\gamma_{5}\right) v_{\nu}
$$

where $\bar{u}_{e}$ and $v_{\nu}$ are the standard Dirac spinors for the electron and the neutrino. Because of possible gluon exchanges at the hadronic vertex, the hadronic current cannot be written down exactly. Utilizing all the available four-vectors in the decay $p_{D}$ and $p_{K}$, the momenta of the $D^{+}$and the $\bar{K}^{* 0}$, and $\epsilon$, the polarization vector of the $\bar{K}^{* 0}$ - and the requirement that each term must be linear in $\epsilon$, we get the following expression for the hadronic current:

$$
\begin{aligned}
\mathcal{H}_{\mu} & =\left(M_{D}+M_{K^{*}}\right) A_{1}\left(q^{2}\right) \epsilon_{\mu}-\frac{A_{2}\left(q^{2}\right)}{M_{D}+M_{K^{*}}}\left(\epsilon \cdot p_{D}\right)\left(p_{D}+p_{K}\right)_{\mu} \\
& -\frac{A_{3}\left(q^{2}\right)}{M_{D}+M_{K^{*}}}\left(\epsilon \cdot p_{D}\right)\left(p_{D}-p_{K}\right)_{\mu}-i \frac{2 V\left(q^{2}\right)}{M_{D}+M_{K^{*}}} \varepsilon_{\mu \nu \rho \sigma} \epsilon^{\nu} p_{D}^{\rho} p_{K}^{\sigma}
\end{aligned}
$$


where $q^{2}$ is the square of the invariant mass of the virtual $W^{+} . \mathcal{H}_{\mu}$ is thus parameterized by four Lorentz invariant form factors, $A_{1}\left(q^{2}\right), A_{2}\left(q^{2}\right), A_{3}\left(q^{2}\right)$, and $V\left(q^{2}\right)$, which represent the axial and vector components of the electroweak current. In the limit of zero lepton mass, $\left(p_{D}-p_{K}\right)_{\mu} \mathcal{L}^{\mu}=0$; thus the $A_{3}\left(q^{2}\right)$ term is not significant. Hence we are left with three terms describing the decay. The functional form of the form factors is not known, and cannot be calculated exactly. Several models estimate the form factors at specific values of $q^{2}$. In this analysis, we use the observed correlations between the final-state particles in the decay $D^{+} \rightarrow \bar{K}^{* 0} e^{+} \nu_{e}$ to measure the form factor ratios $r_{2}=\frac{A_{2}(0)}{A_{1}(0)}$ and $r_{V}=\frac{V(0)}{A_{1}(0)}$ which are evaluated at $q^{2}=0$.

\section{$1.2 \quad q^{2}$ Dependence}

The allowed range of $q^{2}$ in $D^{+} \rightarrow \bar{K}^{* 0} e^{+} \nu_{e}$ decays is

$$
\left(q_{\min }^{2}=m_{e}^{2} \approx 0\right) \leq q^{2} \leq\left(q_{\max }^{2}=\left[M_{D}-M_{K^{*}}\right]^{2}\right)
$$

but, as stated before, the dependence of the form factors on $q^{2}$ is not known. Many theoretical models assume some form of nearest-pole dominance. It is assumed that a $c$ quark would be more likely to couple to an $s$ quark near a $c \bar{s}$ resonance. The $W^{+}$is a spin 1 particle. Thus, only resonant particles with spin 1 and appropriate parity can be pole candidates. The form factors $A_{1}\left(q^{2}\right)$ and $A_{2}\left(q^{2}\right)$ characterize the axial vector part of the hadronic current. The least massive $J^{P}=1^{+}$state is the $D_{s 1}^{*}$ at $2.5 \mathrm{GeV} .{ }^{1}$ The vector part is characterized by $V\left(q^{2}\right)$. The appropriate pole for the $1^{-}$state is the $D_{s}^{*}$ at $2.1 \mathrm{GeV}$. The $q^{2}$ dependence, assumed in this analysis, is expressed as

$$
F\left(q^{2}\right)=\frac{F(0)}{1-q^{2} / m_{\text {pole }}^{2}}
$$

where $F$ stands for any form factor, and $m_{\text {pole }}$ is the relevant pole mass discussed above.

\footnotetext{
${ }^{1}$ Throughout this document we use "natural units" where $\hbar=c=1$. Mass, energy, and momentum are then measured in $\mathrm{GeV}$.
} 


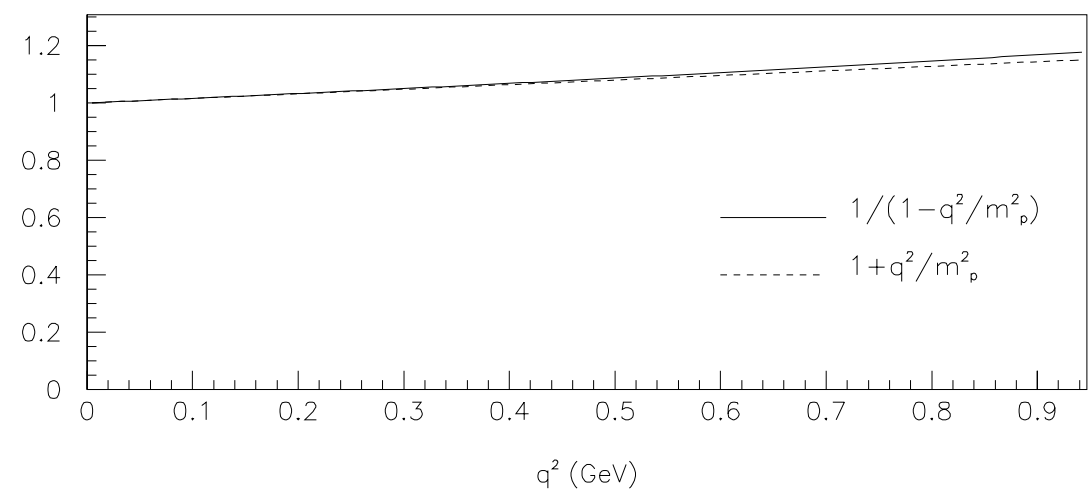

Figure 1.2: Two functional forms of the $q^{2}$ dependence of the form factors for the allowed range of $q^{2}$ in the decay $D^{+} \rightarrow \bar{K}^{* 0} e^{+} \nu_{e}$ with $m_{p}=2.5 \mathrm{GeV}$.

Another possible parameterization of the $q^{2}$ dependence is the linear form given by

$$
F\left(q^{2}\right)=F(0)\left(1+\rho q^{2}\right)
$$

where $\rho$ is the slope. In the $q^{2}$ range relevant in the $D^{+} \rightarrow \bar{K}^{* 0} e^{+} \nu_{e}$ decay $(0-0.947$ $\left.\mathrm{GeV}^{2}\right)$, Eq. 1.6 is a fair approximation of Eq. 1.5 with $\rho=1 / m_{\text {pole }}^{2}$ as can be seen in Figure 1.2.

\subsection{Differential Decay Rate}

We extract the form factor ratios from the data by measuring the differential decay rate of the $D^{+} \rightarrow \bar{K}^{* 0} e^{+} \nu_{e}$ process. We describe the decay as a succession of 2-body decays. First the $D^{+}$decays into a $\bar{K}^{* 0}$ and a virtual $W^{+}$. Next $\bar{K}^{* 0}$ decays into a $K^{-}$and a $\pi^{+}$, and the $W^{+}$into a $e^{+}$and a $\nu_{e}$. There are five measurable kinematic parameters in the decay: $M_{K \pi}$, the invariant mass of the $\bar{K}^{* 0}$ decay products; $q^{2}$, the square of the invariant mass of the virtual $W^{+} ; \theta_{l}$, the angle between the lepton, and the direction opposite the recoiling $D^{+}$in the rest frame of the $W^{+} ; \theta_{V}$, the angle between the kaon and the direction opposite that of the recoiling $D^{+}$, in the 


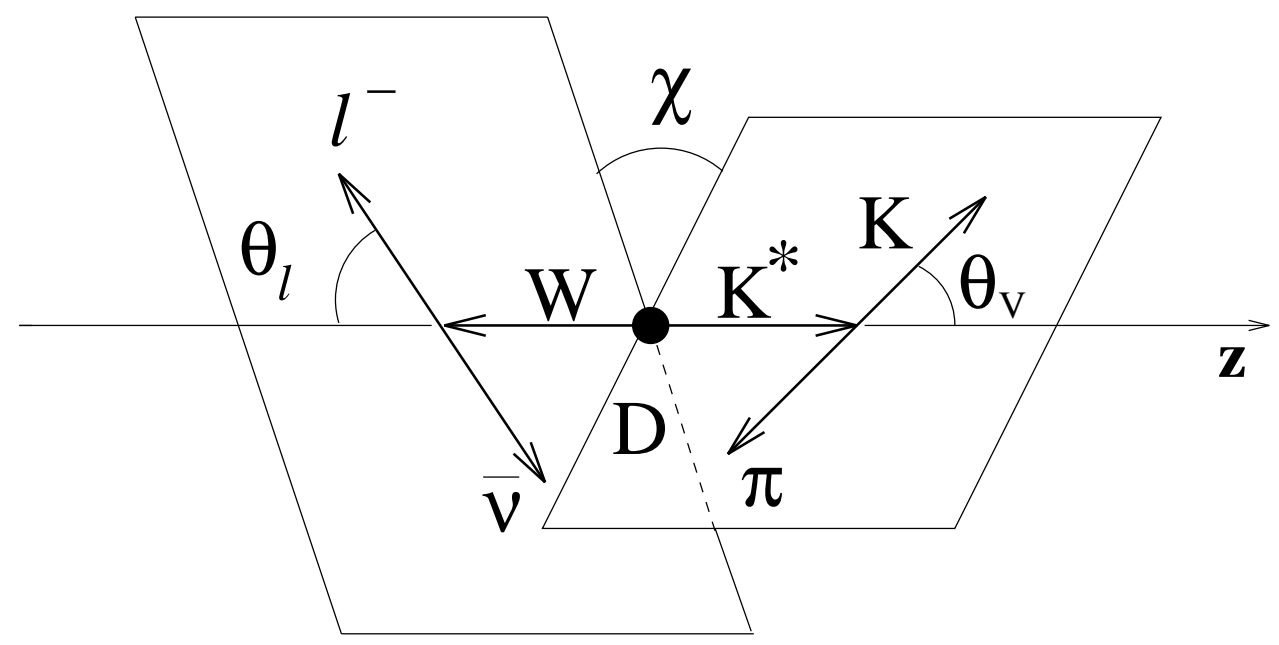

Figure 1.3: Definition of the kinematic angles $\theta_{l}, \theta_{V}$, and $\chi$

rest frame of the $\bar{K}^{* 0}$; and $\chi$, the angle between the directions of the kaon and the electron in the plane perpendicular to the $\bar{K}^{* 0}$ and $W^{+}$directions in the $D^{+}$rest frame. Figure 1.3 shows the three decay angles. In this diagram the directions of the final-state particles, $l^{-}, \bar{\nu}, K$, and $\pi$, are shown in the rest frame of the parent particles $W$ and $K^{*}$, respectively.

In terms of the five kinematic variables, the differential decay rate is written as follows:

$$
\begin{aligned}
\frac{d,}{d M_{K \pi}^{2} d q^{2} d \cos \theta_{V} d \cos \theta_{l} d \chi} & =\frac{1}{2} G_{F}^{2}\left|V_{c s}\right|^{2}\left|\mathcal{L}^{\mu} \mathcal{H}_{\mu}\right|^{2} \\
& =G_{F}^{2}\left|V_{c s}\right|^{2} \frac{3}{2(4 \pi)^{5}} \frac{M_{K^{*}}}{M_{D}^{2} M_{K \pi}} p_{K} q^{2} \\
& \times \frac{M_{K^{*}},\left(M_{K \pi}\right)}{\left(M_{K \pi}^{2}-M_{K^{*}}^{2}\right)^{2}+M_{K^{*}}^{2},{ }^{2}\left(M_{K \pi}\right)} \\
& \times\left\{\left(1+\cos \theta_{l}\right)^{2}\left|H_{+}\left(q^{2}\right)\right|^{2} \sin ^{2} \theta_{V}\right. \\
& +\left(1-\cos \theta_{l}\right)^{2}\left|H_{-}\left(q^{2}\right)\right|^{2} \sin ^{2} \theta_{V} \\
& +4 \sin ^{2} \theta_{l} \cos ^{2} \theta_{V}\left|H_{0}\left(q^{2}\right)\right|^{2} \\
& -2 \sin ^{2} \theta_{l} \sin ^{2} \theta_{V} \operatorname{Re}\left(e^{i 2 \chi} H_{+}^{*} H_{-}\right) \\
& -4 \sin _{l} \theta_{l}\left(1+\cos \theta_{l}\right) \sin \theta_{V} \cos \theta_{V} \operatorname{Re}\left(e^{i \chi} H_{+}^{*} H_{0}\right)
\end{aligned}
$$




$$
\left.+\quad 4 \sin \theta_{l}\left(1-\cos \theta_{l}\right) \sin \theta_{V} \cos \theta_{V} \operatorname{Re}\left(e^{i \chi} H_{-}^{*} H_{0}\right)\right\}
$$

The form factors are contained within the helicity amplitudes $H_{ \pm}$and $H_{0}$, which correspond to the transverse and longitudinal helicities of the $W^{+}$or the vector meson $\bar{K}^{* 0}$. Since the $D^{+}$is a pseudoscalar, it has spin 0; thus its two-body decay products $\left(W^{+}\right.$and $\left.\bar{K}^{* 0}\right)$ must have the same helicity. The helicity amplitudes are given by

$$
\begin{aligned}
H_{ \pm}\left(q^{2}\right)= & \left(M_{D}+M_{K \pi}\right) A_{1}\left(q^{2}\right) \mp 2 \frac{M_{D} p_{K}}{M_{D}+M_{K \pi}} V\left(q^{2}\right) \\
H_{0}\left(q^{2}\right)= & \frac{1}{2 q M_{K \pi}}\left[\left(M_{D}^{2}-M_{K \pi}^{2}-q^{2}\right)\left(M_{D}+M_{K \pi}\right) A_{1}\left(q^{2}\right)\right. \\
& \left.-4 \frac{M_{D}^{2} p_{K}^{2}}{M_{D}+M_{K \pi}} A_{2}\left(q^{2}\right)\right] .
\end{aligned}
$$

$V\left(q^{2}\right)$ contributes only to the transverse helicity amplitudes, $H_{ \pm}$, whereas $A_{2}\left(q^{2}\right)$ contributes only to $H_{0}\left(q^{2}\right) . A_{1}\left(q^{2}\right)$ contributes to all three, and is the dominant form factor at high $q^{2}$. Since $A_{1}\left(q^{2}\right)$ contributes to all three, it can be factored out of each helicity amplitude. Then $H_{ \pm}$and $H_{0}$ can be expressed in terms of $A_{1}$ and the form factor ratios $r_{2}=\frac{A_{2}}{A_{1}}$ and $r_{V}=\frac{V}{A_{1}}$. The vector form-factor ratio $r_{V}$ is most sensitive to the $\cos \theta_{l}$ distribution and the correlation between $\cos \theta_{V}$ and $\chi$. The axial form-factor ratio $r_{2}$ is very sensitive to the distribution of $q^{2} . M_{K \pi}$ distribution provides no information on the form-factor ratios. It is used primarily for background discrimination. Appendix A discusses the distributions of the differential decay rate in the space of each pair of two of the other four kinematic variables, integrating over the remaining two. For a more thorough discussion of the semileptonic decays of charm hadrons see Reference [2].

\subsection{Heavy Quark Effective Theory}

Recent work in the field of heavy quark physics [3] has led to an expansion of knowledge and understanding of the physics involved in semileptonic decays of mesons containing a heavy quark, such as $D^{ \pm}$. The six known quarks fall into two categories: 
heavy $(c, b$, and $t)$ and light $(u, d$, and $s)$. The heavy quarks are much more massive than the QCD parameter $\Lambda_{Q C D}$ which comes from the equation describing the running of the strong coupling constant [4]

$$
\alpha_{s}(\mu) \approx \frac{4 \pi}{\left(11-\frac{2}{3} n_{f}\right) \ln \left(\mu^{2} / \Lambda^{2}\right)}
$$

where $\mu$ is the energy scale, and $n_{f}$ is the number of quarks with mass less than $\mu$. The value of $\Lambda_{Q C D}$ is on the order of $200-400 \mathrm{MeV}$, much higher than the masses of the light quarks $u$ and $d$ and of the same order as the mass of the $s$ quark. The well-known light quark SU(3)-flavor symmetry comes from the limit of $m_{q_{i}} \rightarrow 0^{2}$. In a similar manner we can take the opposite limit of $m_{Q_{i}} \rightarrow \infty$. This regime gives valuable insight into the physics of heavy hadrons, and through the Heavy Quark Effective Theory (HQET) it has been shown that QCD remains well-behaved [3].

The main consequence prescribed by the HQET is that the heavy quark within a hadron can be seen as a static source of a color field. Its four-velocity $v_{\mu}$ decouples from the "light degrees of freedom" (the light quarks, gluons, and their spins). The four-velocity is defined as

$$
v_{\mu}=\frac{p_{\mu}}{m_{Q_{i}}}=(\gamma, \gamma \vec{v}) ; \quad v_{\mu} v^{\mu}=\gamma^{2}\left(1-\beta^{2}\right)=1
$$

Thus a heavy quark in a hadron can be replaced by another heavy quark at the same four-velocity $v$, but not necessarily the same spin, and the configuration of the light degrees of freedom is not affected. In addition the velocity of the hadron is taken to be the velocity of the heavy quark [2].

Since in HQET the four-velocity is the only quantity needed to describe the state of a heavy meson, HQET describes the behavior of form factors in the semileptonic decay $P \rightarrow V \ell \nu$ in terms of the four-velocity transfer $\left(v-v^{\prime}\right)^{2}$ instead of the fourmomentum transfer $q^{2}$. We define the quantity $w$

$$
w \equiv v \cdot v^{\prime}=\frac{m_{P}^{2}+m_{V}^{2}-q^{2}}{2 m_{P} m_{V}} \geq 1
$$

\footnotetext{
${ }^{2}$ We use the notation $q_{i}$ and $Q_{i}$ to denote light and heavy quarks, respectively.
} 
where $m_{P}$ and $v$ are the mass and the four-velocity of the decaying pseudoscalar meson, and $m_{V}$ and $v^{\prime}$ are the mass and the four-velocity of the vector meson to which it decays. In terms of $w$ the hadronic current for $P \rightarrow V \ell \nu$ is

$$
\begin{aligned}
\mathcal{H}_{\mu}= & \sqrt{m_{P} m_{V}}\left[i h_{V}(w) \varepsilon_{\mu \nu \alpha \beta} \epsilon^{\nu} v^{\prime \alpha} v^{\beta}-h_{A_{1}}(w) \epsilon_{\mu}(w+1)\right. \\
& \left.+h_{A_{2}}(w) v_{\mu} \epsilon \cdot v+h_{A_{3}}(w) v_{\mu}^{\prime} \epsilon \cdot v\right]
\end{aligned}
$$

where the HQET form factors are $h_{V}, h_{A_{1}}, h_{A_{2}}$, and $h_{A_{3}}$. They are related to the standard set of form factors in Eq. 1.3 by

$$
\begin{aligned}
V\left(q^{2}\right) & =\left(\frac{m_{P}+m_{V}}{2 \sqrt{m_{P} m_{V}}}\right) h_{V}(w), \\
A_{1}\left(q^{2}\right) & =\left(\frac{2 \sqrt{m_{P} m_{V}}}{m_{P}+m_{V}}\right) \frac{w+1}{2} h_{A_{1}}(w), \\
A_{2}\left(q^{2}\right) & =\left(\frac{m_{P}+m_{V}}{2 \sqrt{m_{P} m_{V}}}\right)\left[h_{A_{3}}(w)+\frac{m_{V}}{m_{P}} h_{A_{2}}(w)\right] .
\end{aligned}
$$

In the limit of heavy quark mass symmetry $\left(m_{Q_{P}}, m_{Q_{V}} \rightarrow \infty\right)$ HQET predicts that

$$
h_{V}(w)=h_{A_{1}}(w)=h_{A_{3}}(w)=\xi(w) ; \text { and } h_{A_{2}}(w)=0
$$

where $\xi(w)$ is the Isgur-Wise function, a universal form factor. Of particular interest is the kinematic point of zero recoil at $q_{\max }^{2}$, or $w=1$. Here $Q_{P}$ decays into $Q_{V}$, which remains stationary in the reference frame of $Q_{P}$, and the lepton and neutrino fly out back-to-back. The initial-state and final-state wave functions remain essentially identical. The Isgur-Wise function is then normalized to $\xi(1)=1$.

Since the quark masses are not degenerate, heavy quark symmetry is broken. In the case of $B^{-} \rightarrow D^{* 0} \ell^{-} \bar{\nu}$ both $Q_{P}(b)$ and $Q_{V}(c)$ are heavy, and HQET is expected to hold approximately, up to corrections of order $1 / m_{Q}$. This is not the case in the decay $D^{+} \rightarrow \bar{K}^{* 0} e^{+} \nu_{e}$ since the $s$ quark in $\bar{K}^{* 0}$ is not heavy, and heavy quark symmetry is badly broken. The corrections to HQET predictions are expected to be very large. More work is needed in calculating the corrections to the predictions for the form factors in semileptonic charm decay. 


\subsubsection{Form Factors in Bottom Decays}

One interesting prediction of HQET is that form factors in semileptonic charm decay can be related to those in bottom decays at the same four-velocity transfer near $q^{2}=q_{\max }^{2}[5]$. In the limit of heavy quark symmetry where the mass of the heavy quark in $D$ or $\bar{B}$ ( $c$ and $b$, respectively) mesons approaches infinity, the $c \rightarrow s$ transition in $D^{+}$semileptonic decay is analogous to the $b \rightarrow s$ transition in rare $B$ decays, such as $\bar{B}^{0} \rightarrow K^{* 0} e^{+} e^{-}$. The form factors that parameterize each process can then be related in the following manner:

$$
\begin{aligned}
\left(a_{+}+a_{-}\right)^{\left(\bar{B} \rightarrow K^{*}\right)} & =\left[\frac{m_{c}}{m_{b}}\right]^{3 / 2}\left[\frac{\alpha_{s}\left(m_{b}\right)}{\alpha_{s}\left(m_{c}\right)}\right]^{-6 / 25}\left(a_{+}+a_{-}\right)^{\left(D \rightarrow K^{*}\right)} \\
\left(a_{+}-a_{-}\right)^{\left(\bar{B} \rightarrow K^{*}\right)} & =\left[\frac{m_{c}}{m_{b}}\right]^{1 / 2}\left[\frac{\alpha_{s}\left(m_{b}\right)}{\alpha_{s}\left(m_{c}\right)}\right]^{-6 / 25}\left(a_{+}-a_{-}\right)^{\left(D \rightarrow K^{*}\right)}, \\
g^{\left(\bar{B} \rightarrow K^{*}\right)} & =\left[\frac{m_{c}}{m_{b}}\right]^{1 / 2}\left[\frac{\alpha_{s}\left(m_{b}\right)}{\alpha_{s}\left(m_{c}\right)}\right]^{-6 / 25} g^{\left(D \rightarrow K^{*}\right)}, \\
f^{\left(\bar{B} \rightarrow K^{*}\right)} & =\left[\frac{m_{c}}{m_{b}}\right]^{1 / 2}\left[\frac{\alpha_{s}\left(m_{b}\right)}{\alpha_{s}\left(m_{c}\right)}\right]^{-6 / 25} f^{\left(D \rightarrow K^{*}\right)},
\end{aligned}
$$

where $m_{c}$ and $m_{b}$ are the quark masses and $\alpha_{s}$ is the running QCD coupling constant. The form factors $f, g, a_{+}$, and $a_{-}$are related to the standard form factors in the following manner:

$$
\begin{gathered}
A_{1}=\frac{f}{M_{D}+M_{K^{*}}}, \quad A_{2}=-\left(M_{D}+M_{K^{*}}\right) a_{+}, \\
A_{3}=\frac{q^{2}}{2 M_{K^{*}}}\left(a_{+}-a_{-}\right), \quad V=\left(M_{D}+M_{K^{*}}\right) g .
\end{gathered}
$$

In the limit of light-quark $\mathrm{SU}(3)$-flavor symmetry, where $u, d$, and $s$ quarks are just different isospin states of the same particle, the $\bar{B} \rightarrow K^{*}$ form factors are simply related to the $\bar{B} \rightarrow \rho$ form factors by Clebsch-Gordan coefficients. Using Eqs. 1.4 and 1.11 we calculate the ranges of $q^{2}$ and $w$ accessible in these decays. They are listed in Table 1.1. The relations given by Eq. 1.15 are applicable in the region near $q_{\text {max }}^{2}$ (near $w=1$ ). Thus by using the measured form factors in semileptonic $D$ decays 


\begin{tabular}{|l|c|c|}
\hline Decay & $q^{2}$ range $\left(\mathrm{GeV}^{2}\right)$ & $w$ range \\
\hline$D^{+} \rightarrow \bar{K}^{* 0} e^{+} \nu_{e}$ & $0-0.947$ & $1-1.28$ \\
$\bar{B}^{0} \rightarrow K^{* 0} e^{+} e^{-}$ & $0-19.2$ & $1-3.03$ \\
$B^{+} \rightarrow \rho^{0} e^{+} \nu_{e}$ & $0-20.2$ & $1-3.36$ \\
\hline
\end{tabular}

Table 1.1: Accessible $q^{2}$ and $w$ ranges in $D$ and $B$ decays.

and the technique outlined above to derive the form factors in $B$ decays, as well as the experimental measurement of,$\left(B^{+} \rightarrow \rho e^{+} \nu_{e}\right)$, the CKM matrix element $V_{u b}$ can be extracted.

\subsection{Theoretical Predictions}

Several theoretical models make predictions for the form factor ratios $r_{2}$ and $r_{V}$, which are summarized in Table 1.2. In addition the predictions for the individual form factors measured at $q^{2}=0$ and $q^{2}=q_{\text {max }}^{2}$ are listed in Table 1.3. The theoretical models fall into three categories: quark models, lattice gauge calculations, and QCD sum rules.

The quark models use estimates of the meson wave function to calculate the matrix elements in the hadronic currents at a particular value of $q^{2}$. Each model assumes a particular form of the $q^{2}$ dependence. The ISGW model [6], as well as its updated version ISGW2 [7], computes the form factors at $q_{\max }^{2}$ using the following $q^{2}$ dependence:

$$
F\left(q^{2}\right)=F\left(q_{\max }^{2}\right) \exp \left[-0.03 \mathrm{GeV}^{-2}\left(q_{\max }^{2}-q^{2}\right)\right] .
$$

The ISGW2 model fully incorporates heavy quark symmetry in its calculation. The other models calculate the form factors at $q^{2}=0$ using a pole form for the $q^{2}$ dependence

$$
F\left(q^{2}\right)=\frac{F(0)}{\left(1-q^{2} / m_{\text {pole }}^{2}\right)^{n}}
$$

where $n=1$ in the WSB [8] and AW/GS [9, 10] models, and $n=2$ in the KS [11] model. A recent quark model calculation by Stech [12] makes an assumption for the 
$q^{2}$ (or $w$ ) dependence of the Isgur-Wise function that respects "scaling and analyticity requirements", and is expressed in terms of the initial and final state meson masses, as well as the mass of a pole with the appropriate quantum numbers. The results of this calculation for the form factors $A_{1}(0), A_{2}(0)$, and $V(0)$ are listed in Table 1.3.

Lattice gauge calculations provide a nonperturbative, numerical solution of QCD [13]. Major sources of systematic error are due to the discrete nature of the lattice and the size of the lattice spacing. A pole form for the $q^{2}$ dependence is assumed. QCD sum rules provide a methodology for calculating form factors, which is more directly related to field theory than are quark models. Results of a sum rule calculation [14] are listed in Table 1.2, in which the authors compute the contributions to the three-point function, which defines the hadronic matrix element, from various possible sources.

There are, however, large uncertainties on all these predictions. The experimental average calculated from three previously published results $[15,16,17]$ is also listed in Tables 1.2 and 1.3. This average does not include the measurement described in this thesis. 


\begin{tabular}{|l|c|c|}
\hline Group & $r_{2}$ & $r_{V}$ \\
\hline \multicolumn{3}{|c|}{ Quark Models } \\
\hline ISGW [6] & 1.0 & 1.4 \\
WSB [8] & 1.3 & 1.4 \\
KS [11] & 1.0 & 1.0 \\
AW/GS [9, 10] & 0.8 & 2.0 \\
\hline \multicolumn{3}{|c|}{ Lattice Gauge } \\
\hline BKS [18] $0.7 \pm 0.16 \pm 0.17$ & $1.99 \pm 0.22 \pm 0.33$ \\
LMMS [19] & $0.4 \pm 0.4$ & $1.6 \pm 0.2$ \\
LANL [20] & $0.68 \pm 0.11$ & $1.78 \pm 0.07$ \\
ELC [21] & $0.6 \pm 0.3$ & $1.3 \pm 0.2$ \\
APE [22] & $0.7 \pm 0.4$ & $1.6 \pm 0.3$ \\
UKQCD [23, 24] & $0.9 \pm 0.2$ & $1.4_{-0.2}^{+0.5}$ \\
\hline \multicolumn{3}{|c|}{ Sum Rules } \\
\hline BBD [14] & $1.2 \pm 0.2$ & $2.2 \pm 0.2$ \\
\hline \hline Experimental Average & $0.73 \pm 0.15$ & $1.89 \pm 0.25$ \\
\hline
\end{tabular}

Table 1.2: Theoretical predictions and experimental average for the form factor ratios $r_{2}$ and $r_{V}$. The experimental average does not include the results of this analysis.

\begin{tabular}{|l|c|c|c|}
\hline Group & $A_{1}(0)$ & $A_{2}(0)$ & $V(0)$ \\
\hline APE [22] & $0.67 \pm 0.11$ & $0.49 \pm 0.34$ & $1.08 \pm 0.22$ \\
Wuppertal [25] & $0.61_{-0.09}^{+0.11}$ & $0.83_{-0.22}^{+0.23}$ & $1.34_{-0.28}^{+0.31}$ \\
UKQCD [23] & $0.70_{-0.10}^{+0.07}$ & $0.66_{-0.15}^{+0.10}$ & $1.01_{-0.13}^{+0.30}$ \\
ELC [21] & $0.64 \pm 0.16$ & $0.41 \pm 0.28 \pm 0.04$ & $0.86 \pm 0.24$ \\
Stech [12] & 0.69 & 0.73 & 1.07 \\
\hline Experimental Average & $0.56 \pm 0.04$ & $0.39 \pm 0.08$ & $1.1 \pm 0.2$ \\
\hline \hline & $A_{1}\left(q_{\text {max }}^{2}\right)$ & $A_{2}\left(q_{\text {max }}^{2}\right)$ & $V\left(q_{\text {max }}^{2}\right)$ \\
\hline ISGW2 [7] & 0.70 & 0.94 & 1.52 \\
\hline Experimental Average & $0.66 \pm 0.05$ & $0.46 \pm 0.09$ & $1.4 \pm 0.3$ \\
\hline
\end{tabular}

Table 1.3: Theoretical predictions for the form factors $A_{1}, A_{2}$, and $V$. The experimental measurements for the form factors are done at $q^{2}=0$ and extrapolated to $q_{\max }^{2}$ using the pole form of the $q^{2}$ dependence given by Eq. 1.5. Experimental average does not include the results of this analysis. 


\section{Chapter 2}

\section{E791 Experiment}

\section{$2.1 \quad$ Overview}

The E791 fixed target experiment was conducted at the Fermi National Accelerator Laboratory in Batavia, Illinois between July 1991 and January 1992. Over 20 billion interactions of a $500 \mathrm{GeV} \pi^{-}$beam with five thin target foils (one platinum, and four carbon) were recorded. Upstream of the target, protons in the 1.5 mile diameter Main Ring were directed toward the experimental area, where interactions with a $30 \mathrm{~cm}$ long Beryllium target produced the $\pi^{-}$beam. Events produced in the $\pi^{-}$ nucleon interactions in the target foils were recorded by the upgraded Tagged Photon Spectrometer, a detector which has been in use since the E516 experiment in 1976. Silicon microstrip detectors, proportional wire chambers, drift chambers, and bending magnets provided vertex and tracking information. Two Čerenkov counters provided hadronic particle identification. Electromagnetic and hadronic calorimeters measured the energies deposited by the charged particles and photons and also aided in particle identification. A wall of scintillating paddles detected the highly penetrating muons. Figure 2.1 shows a diagram of the spectrometer. See References [26, 27] for a thorough description of the Tagged Photon Spectrometer.

A number of facets particular to this experiment produce a large recorded charm event sample; certain aspects of the detector allow us to suppress non-charm backgrounds offline. This combination enables us to make the most precise measurement 
of the $D^{+} \rightarrow \bar{K}^{* 0} e^{+} \nu_{e}$ semileptonic form factors to date. The Data Acquisition System allows E791 to record up to 20 billion events in a six month period by continuously buffering, processing, and writing events to tape during the data-taking run. The Silicon Microstrip Detectors provide improved charged particle tracking so that a detached secondary vertex can be reconstructed. The segmented target structure allows us to distinguish $D^{ \pm}$decays in the space between the target foils from noncharm secondary interactions in the target material thus reducing a major source of background. These systems along with others which are the most relevant to this analysis are explored in further detail in the following sections.

\section{$2.2 \quad$ Target}

The E791 target structure fulfills the need for a large number of interactions, while reducing backgrounds from secondary interactions. Previous experiments, such as E687 [17] which used a $4.5 \mathrm{~cm}$ beryllium block as a target, found high amounts of background from secondary interactions. The E791 target consists of 5 thin foils (one platinum, four carbon) separated by approximately $1.5 \mathrm{~cm}$. Platinum is an appropriate target material for the most upstream foil since the probability that a beam particle interacts in the target is proportional to $A$, the atomic weight of the target material and Platinum has a high atomic weight $(A \approx 195)$. Since the probability for multiple scattering in downstream target material is proportional to $Z^{2}$, the atomic number, Carbon is the more appropriate choice for downstream foils since Carbon has a relatively low atomic number $(Z=6)$. The thickness of the foils was chosen to be approximately $0.4 \%$ of an interaction length in order to maximize the number of primary interactions. It is desirable that a charmed particle produced in the interaction of the $\pi^{-}$beam and a nucleon inside one of the foils decays in space before reaching the next foil. For a typical $D^{+}$lifetime of $1 \times 10^{-12} \mathrm{~s}, \gamma \equiv E / m \approx 30$, and $\beta \approx 1$, the decay length, given by $l=\gamma \beta c \tau$ is about $1 \mathrm{~cm}$. The details of the target assembly are given in Table 2.1. The complete target assembly corresponds to about $2.2 \%$ of an interaction length. 


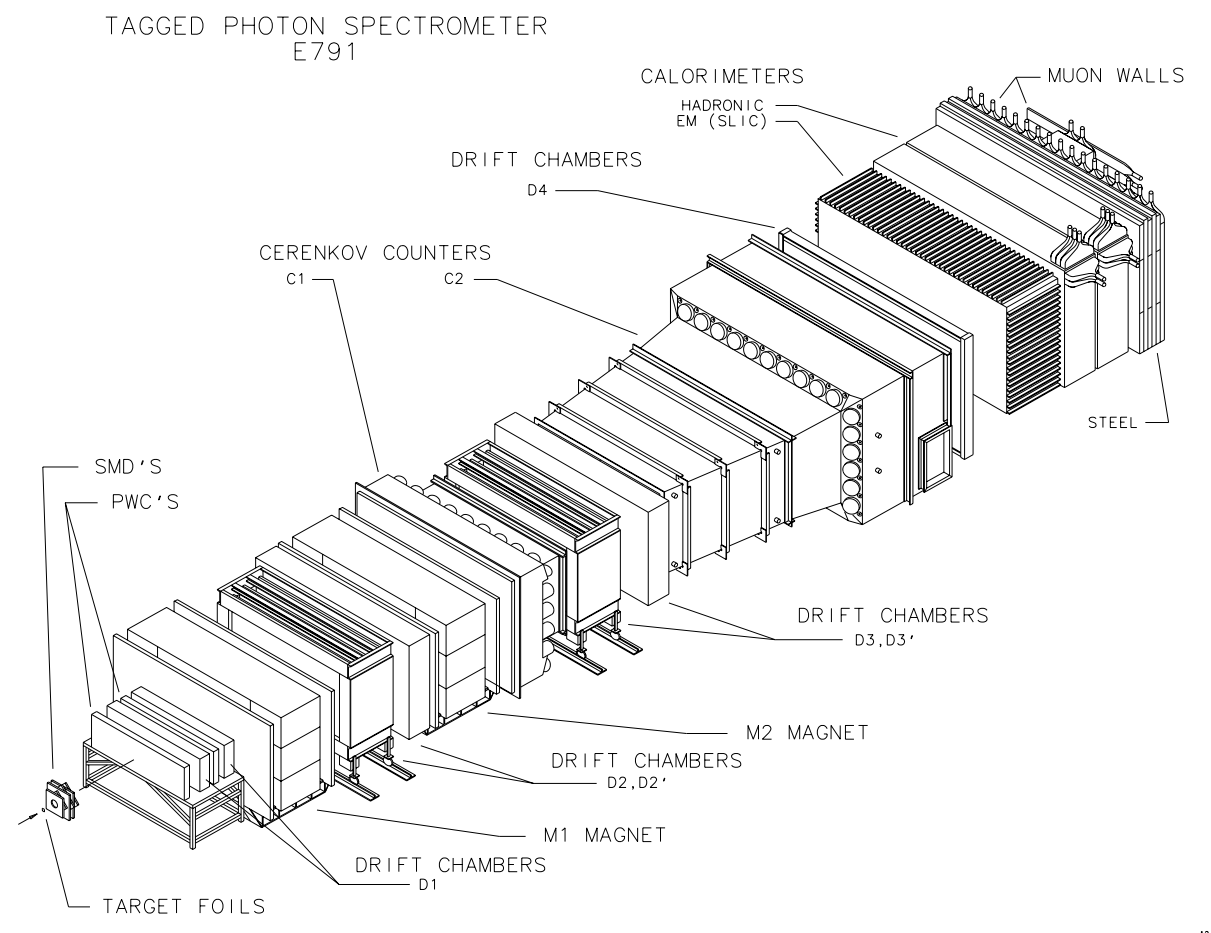

Figure 2.1: The E791 spectrometer.

\begin{tabular}{|l|c|c|c|c|}
\hline Foil & Material & Spacing $(\mathrm{cm})$ & Thickness $(\mathrm{mm})$ & Int. Length $(\%)$ \\
\hline 1 & $\mathrm{Pt}$ & & 0.52 & 0.588 \\
2 & $\mathrm{C}$ & 1.501 & 1.57 & 0.412 \\
3 & $\mathrm{C}$ & 1.536 & 1.57 & 0.412 \\
4 & $\mathrm{C}$ & 1.560 & 1.53 & 0.402 \\
5 & $\mathrm{C}$ & 1.534 & 1.58 & 0.415 \\
\hline
\end{tabular}

Table 2.1: E791 target assembly. 


\subsection{Tracking System}

\subsubsection{Silicon Microstrip Detectors}

In an analysis of decays such as $D^{+} \rightarrow \bar{K}^{* 0} e^{+} \nu_{e}$, it is imperative to be able to reconstruct well both the production and the decay vertices. High precision tracking and vertexing was provided by the Silicon Microstrip Detectors (SMD's) which operate on the following principle. As a charged particle passes through a thin layer of silicon it deposits ionization energy, creating electron-hole pairs which separate in the presence of an applied bias voltage [28, 29]. The liberated charge is then collected by aluminum strips deposited on one side of the silicon layer and amplified by the readout electronics.

The SMD's consist of planes of silicon only $300 \mu \mathrm{m}$ thick to minimize the effects of multiple scattering. The pitch of the strips on each plane was on the order of tens of $\mu \mathrm{m}$. The amount of deposited energy was not measured. Instead we only determined if a particular strip was hit. To minimize the noise from thermal excitation, it was necessary to maintain the temperature around the SMD's near $60^{\circ} \mathrm{F}$. There were 23 planes of SMD's. Six planes upstream of the target helped with the beam finding. Seventeen planes downstream of the target were used for vertexing. The downstream planes were grouped by three, each with a different strip orientation (vertical,horizontal, and $-20^{\circ}$ from the vertical) to remove the ambiguity in the cases when there is more than one track leaving "hits" in the SMD. The typical "hit" resolution was $\approx 15 \mu \mathrm{m}$.

By using the information from the SMD's we can make accurate measurements of the vertex positions. Figure 2.2 shows the measured $z$-coordinate (along the beam direction) of the primary vertices in E791 events. The five target foils are clearly seen, as nearly all events are produced in the $\pi^{-}-N$ collisions in the foils. We determine the primary vertex resolution by fitting the distribution of events in each target foil to a convolution of a Gaussian distribution and a flat distribution over the width of the foil. The standard deviation of the Gaussian distribution is a free parameter in the fit. A detailed fit to the third foil is shown in the inset in the figure. The calculated resolution ranges between $450 \mu \mathrm{m}$ and $240 \mu \mathrm{m}$ and is given next to each 
target foil. Charged particles produced in the upstream foils are scattered as they pass through the foils downstream, thus resulting in poorer resolution. Also, seen in Figure 2.2 is the interaction counter downstream of the targets. The events produced from collisions in the interaction counter were subsequently removed from the data sample since they tended to be more contaminated by background.

\subsubsection{Proportional Wire Chambers}

Proportional Wire Chambers (PWC's) aided in beam tracking and the determination of the position of the primary vertex. Eight planes of PWC's were installed upstream of the target for this purpose. Two additional planes were located downstream of the target and added to E791 tracking capability.

The PWC's consist of a series of high-voltage anode wires evenly spaced between grounded cathode planes. When a charged particle passes through the PWC, it ionizes the gas in the chamber. The electrons drift toward the nearest positively charged wire. Since the electric field increases greatly in the immediate area of the wires, the freed electrons accelerate and ionize more gas atoms, thus creating an "avalanche" effect. Gains of up to $\sim 10^{7}$ are possible for a single passing charged particle depending on the mixture of gases present and the applied voltage [28]. The charge collected by the anode wire is recorded as a "hit" on the wire.

E791 PWC's contain a gas mixture of $82.7 \%$ Argon, $17 \%$ Carbon dioxide, and $0.3 \%$ Freon. There are 64 anode wires in each plane. The orientation of wires in most of the PWC's was in the horizontal and vertical directions. Several upstream planes had wires rotated $-60^{\circ}$ with respect to the vertical direction in order to provide threedimensional information on the position of the hit. For a passing track we measure which wires are hit, but not the electron drift time to each wire. Thus the resolution on the spatial measurement by the PWC is determined solely by the wire spacing divided by $\sqrt{12}$ which is $\approx 300 \mu \mathrm{m}$. 


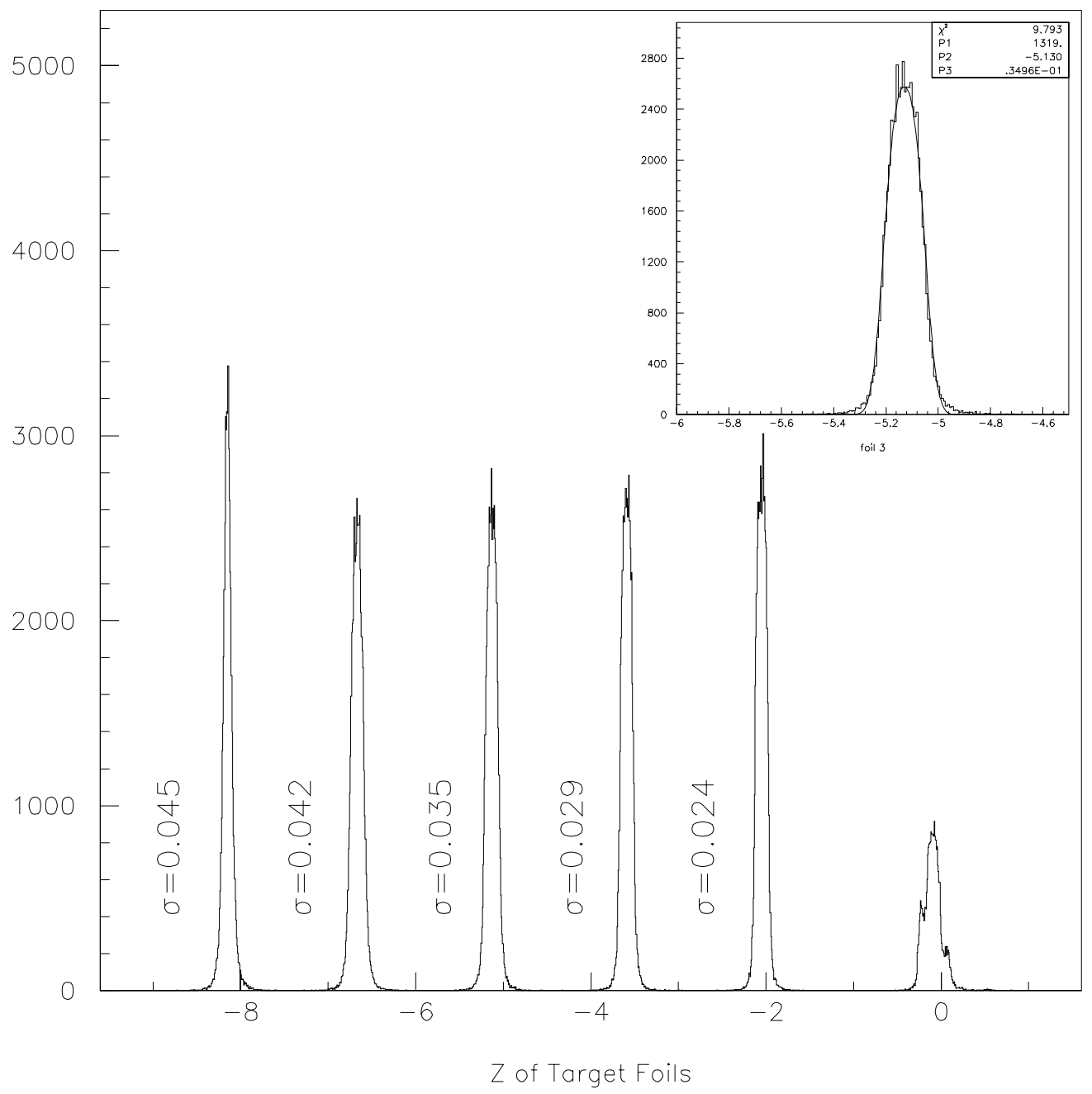

Figure 2.2: The primary vertex position along the beam direction. All lengths are measured in centimeters. The inset shows the fit to the third foil. Details of the fitting technique are described in the text. 


\subsubsection{Drift Chambers}

The Drift Chambers (DC's) operate on a similar principle as the PWC's, namely a charged particle passing through leaves a trail of ions and electrons in its wake. The electrons are collected by positive-voltage wires which are strung through the chamber. Unlike the PWC's the anode sense wires are surrounded by cathode wires. There are additional field wires which maintain the uniformity of the electric field. We measure the drift time of electrons and thus the distance the charged particle passed from the wire. Hence it is possible to measure the actual path of the passing charged track with better precision in the DC's than in the PWC's.

Wires are strung in three different directions (vertical and $\pm 20.5^{\circ}$ from the vertical) in each chamber in order to reconstruct the exact position of the "hit". There are two possible ambiguities that can arise. One is the left-right ambiguity, where it is impossible to tell on which side of the wire the track passed. This is solved by having a second chamber with the same wire orientation, but offset by a small amount. The second type of ambiguity arises when two or more tracks pass through the DC leading to "ghost" tracks. This is solved by a third orientation of wires. The three different views provided by the different wire directions are necessary to resolve the spatial location of each hit. The typical spatial resolution in the DC's is $250 \mu \mathrm{m}$.

Four sets of DC's were used by E791. The first, D1, was located upstream of the first magnet M1. It was comprised of 8 planes of DC's. Being the nearest to the target, these chambers along with the SMD's and the PWC's, provided the initial estimate of track trajectories. The second, D2, was located between the magnets. The third was located directly downstream of the second magnet M2. There were 12 planes of DC's in each set. And finally the fourth set of 3 DC's, D4 was located downstream of the Cerenkov counters. The quality of the measurement of the track trajectory is partially determined by how many DC's the track passes through. An E791 parameter, Category, is assigned to each track based on the number of sets of DC's that have hits associated with it. Category 1 (0001 in binary) refers to a track seen in D1 only. Category 3 (0011) refers to a track in D1 and D2. The maximum value of Category is 15 (1111) referring to a track with hits in all four sets of DC's.

Over time the performance of the DC's degraded in the region nearest the beam 
axis since so many charged particles passed through that small area. Thus at the later stages of data-taking charged tracks passing through the middle of the DC's did not leave as many "hits" on the sense wires, and were thus reconstructed with poorer resolution on the track parameters, or were not reconstructed at all. This analysis should not be affected as long as the degradation of the DC's is properly simulated in the Monte Carlo.

\subsubsection{Magnets}

Two analysis magnets, M1 and M2, play a crucial role in tracking and momentum calculation. When a charged particle passes through a magnetic field, it experiences a force

$$
\mathbf{F}=q \mathbf{v} \times \mathbf{B}
$$

where $q$ is the charge of the particle, $\mathbf{v}$ is the velocity vector, and $\mathbf{B}$ is the magnetic field. Since the magnetic field pointed in the vertical direction, $\mathbf{B}=B \hat{\mathbf{y}}$, and the velocity vector pointed generally in the beam direction, $\mathbf{v} \approx v \hat{\mathbf{z}}$, positively charged tracks were bent to the right in the horizontal plane, and negatively charged tracks were bent to the left, in both magnets. Thus the presence of the magnets allowed us to differentiate between positively and negatively charged tracks.

In the presence of a magnetic field a charged particle follows a helical trajectory as described by Eq. 2.1. The expression for track momentum can be simply derived from this equation:

$$
F=m a=\frac{m v^{2}}{\rho}=q v B, \text { or } p=m v=q B \rho
$$

where $\rho$ is the radius of curvature of the helix. If expressed in standard units for $B$ (Tesla), and $\rho$ (meters), then $p=0.2998 B \rho \mathrm{GeV}$ [28].

E791 magnets were large-aperture copper coil magnets, each approximately $1 \mathrm{~m}$ in length. The current applied to M1 was $2.5 \times 10^{3} \mathrm{~A}$, giving a transverse momentum kick of $212 \mathrm{MeV}$. The magnetic field integrated over the length of M1 was $0.7 \mathrm{~T}-\mathrm{m}$. A current of $1.8 \times 10^{3} \mathrm{~A}$ was applied to M2, giving a transverse momentum kick of 
$320 \mathrm{MeV}$, and the integrated magnetic field was 1.1 T-m [1].

\section{4 Čerenkov Counters}

Two Čerenkov counters are the main tools used for hadronic particle identification in E791. They operate on the fact that a charged particle passing through a dielectric medium with a velocity greater than the speed of light in that medium will radiate [28]. Photons are emitted at a characteristic angle given by

$$
\cos \theta=1 / \beta n
$$

where $\beta=v / c$ is the particle velocity and $n$ is the index of refraction. $\beta_{t h}=1 / n$ represents the minimum (threshold) velocity necessary for a particle to emit photons, since $\cos \theta$ cannot be greater than one. The threshold momentum is then

$$
p_{t h}=\gamma m v_{t h}=\frac{m c}{\sqrt{n^{2}-1}}
$$

where we have used the substitutions, $\beta_{t h}=1 / n$ and $\gamma=\left(1-\beta_{t h}^{2}\right)^{-1 / 2}$. We can then define $\delta=n-1$, and use the fact that $\delta$ is generally very small, to re-express the threshold momentum as

$$
p_{t h}=\frac{m c}{\sqrt{2 \delta}} .
$$

From the above equation its evident that whether a particle with a given momentum radiates depends on its mass and the index of refraction of the material. By using two Čerenkov chambers filled with media with different dielectric properties, over a certain mass range, we can distinguish particles of different mass but the same momentum, which is independently measured by the tracking system. $\mathrm{C} 1$ is filled with Nitrogen gas, which has $\delta=290 \times 10^{-6}$. C2 is filled with $80 \%$ Helium-20\% Nitrogen mixture, which has $\delta=86 \times 10^{-6}$. Table 2.2 lists the momentum ranges for which particle discrimination is possible. Lighter particles, such as pions, will more readily emit photons than heavier particles, such as kaons and protons. 


\begin{tabular}{|c|c|c|c|c|}
\hline $\begin{array}{l}\text { Momentum Range } \\
(\mathrm{GeV})\end{array}$ & $\begin{array}{c}\text { Particle } \\
\text { Type }\end{array}$ & $\begin{array}{l}\text { Radiates } \\
\text { in } \mathrm{C} 1\end{array}$ & $\begin{array}{l}\text { Radiates } \\
\text { in } \mathrm{C} 2 \\
\end{array}$ & $\begin{array}{c}\text { Particle } \\
\text { Discrimination } \\
\end{array}$ \\
\hline $0-6$ & $\begin{array}{l}\pi \\
K \\
p\end{array}$ & & & None \\
\hline $6-11$ & $\begin{array}{l}\pi \\
K \\
p\end{array}$ & $\sqrt{ }$ & & $\pi /(K, p)$ \\
\hline $11-20$ & $\begin{array}{l}\pi \\
K \\
p\end{array}$ & $\sqrt{ }$ & $\sqrt{ }$ & $\pi /(K, p)$ \\
\hline $20-36$ & $\begin{array}{l}\pi \\
K \\
p\end{array}$ & $\begin{array}{l}\sqrt{ } \\
\sqrt{ }\end{array}$ & $\sqrt{ }$ & $\pi / K / p$ \\
\hline $36-38$ & $\begin{array}{l}\pi \\
K \\
p\end{array}$ & $\begin{array}{l}\sqrt{ } \\
\sqrt{ }\end{array}$ & $\begin{array}{l}\sqrt{ } \\
\sqrt{ }\end{array}$ & $(\pi, K) / p$ \\
\hline $38-69$ & $\begin{array}{c}\pi \\
K \\
p\end{array}$ & $\begin{array}{l}\sqrt{ } \\
\sqrt{ } \\
\sqrt{ }\end{array}$ & $\begin{array}{l}\sqrt{ } \\
\sqrt{ }\end{array}$ & $(\pi, K) / p$ \\
\hline$>69$ & $\begin{array}{l}\pi \\
K \\
p\end{array}$ & $\begin{array}{l}\sqrt{ } \\
\sqrt{ } \\
\sqrt{ }\end{array}$ & $\begin{array}{l}\sqrt{ } \\
\sqrt{ } \\
\sqrt{ }\end{array}$ & None \\
\hline
\end{tabular}

Table 2.2: Momentum ranges for which the Čerenkov counters can discriminate between different types of particles. 


\begin{tabular}{|c|c|}
\hline $\begin{array}{c}\text { Particle } \\
\text { Type }\end{array}$ & $\begin{array}{c}\text { a priori } \\
\text { Probability }\end{array}$ \\
\hline$e$ & 0.02 \\
$\mu$ & 0.01 \\
$\pi$ & 0.81 \\
$K$ & 0.12 \\
$p$ & 0.04 \\
\hline
\end{tabular}

Table 2.3: The a priori probability of each particle type in a typical E791 event

An array of mirrors and photomultiplier tubes collect all the photons in the lighttight Čerenkov counters that are produced by passing tracks. Knowing the expected number of photons for, and the approximate preponderance of each type of particle, we calculate the probability for a track with given momentum to be a particular particle. The probability that $n$ photons are detected in the counter $i$ if the particle is of type $\alpha$ is given by the Poisson probability distribution,

$$
f_{\alpha, i}=\frac{\mu^{n} e^{-\mu}}{n !}
$$

where $\mu$ is the predicted number of photons for particle type $\alpha$. To combine the measurements from both counters, $\mathrm{C} 1$ and $\mathrm{C} 2$, we multiply the individual probabilities, $f_{\alpha}=f_{\alpha, 1} \times f_{\alpha, 2}$. Next, we incorporate the a priori expectation of finding particle type $\alpha$ in a typical E791 event, to give the probability $P_{\alpha}$ that the track is of type $\alpha$,

$$
P_{\alpha}=\frac{f_{\alpha} A_{\alpha}}{\sum_{k=1}^{5} f_{k} A_{k}}
$$

where $A_{k}$ is the a priori probability for particle of type $k$ as given in Table 2.3. The five least massive, and most abundant, particles are considered in the calculation of $P_{\alpha}$. The ability of this algorithm to distinguish between different particles is not affected by the values chosen for $A_{k}$. 


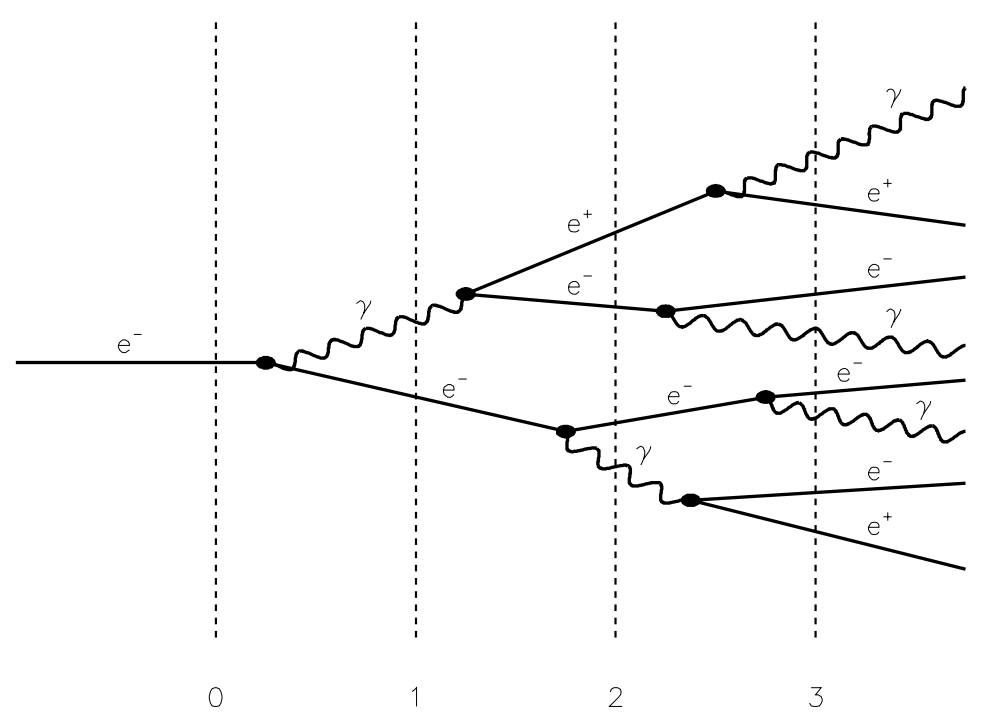

Figure 2.3: Diagram of a typical electromagnetic shower in the SLIC. The distances indicated on the bottom are measured in radiation lengths. Adapted from [28].

\subsection{Electromagnetic Calorimeter}

The electromagnetic calorimeter, called the Segmented Liquid Ionization Calorimeter (SLIC), measured the total energy of photons and electrons (and positrons, which behave in an identical manner) produced in E791 events. As electrons pass through dense material they decelerate and radiate photons. This process is known as bremsstrahlung, a German word meaning "braking radiation". Bremsstrahlung is the chief means by which electrons of energies over $100 \mathrm{MeV}$ lose energy [28]. The radiated photons carry off much of the electron's initial energy. They, in turn, undergo the pair production process whereby an electron-positron pair is created. These particles, in turn radiate, and the process continues until the resultant electrons or photons no longer have enough energy to radiate or pair-produce, respectively. This process is known as an electromagnetic shower, and is illustrated in Figure 2.3.

A completely general description of a shower cannot be calculated. An electron is assumed to travel a distance of one radiation length before giving up all but 1/e 
of its energy by radiation. The number of resulting particles produced in the shower is directly proportional to the incident electron energy. Therefore by detecting the electrons and photons the SLIC measures the initial electron energy.

The SLIC consists of 60 thin slabs of lead interspersed with layers of scintillating liquid. The electrons and photons shower in the lead, and produce ultra-violet photons in the liquid scintillator. Each layer of lead corresponds to approximately 1/3 of a radiation length. The scintillator is separated into channels by teflon-coated corrugated aluminum with total internally-reflecting surfaces. The aluminum channels are oriented in three directions (horizontal, and $\pm 20.5^{\circ}$ from the vertical). The spatial resolution is about $7 \mathrm{~mm}$ for the position of each shower in an event. The UV photons produced in the scintillator propagated to the edge of the SLIC and were collected and counted by photomultiplier tubes. All photons from channels with the same orientation were collected by a single light pipe and carried to a single photomultiplier tube.

The variable EMPROB is used for electron identification. A quantity that can be used to discriminate electrons from pions is calculated based on the following: the amount of energy deposited by the track in the electromagnetic calorimeter, the transverse distribution of the deposited energy, the distance of the centroid of the electromagnetic shower to the intersection of the track with the calorimeter, and the energy associated with the track in the hadronic calorimeter. EMPROB is defined as the probability (in \%) that a particle is an electron, in a beam with equal numbers of electrons and pions, and is given by

$$
E M P R O B=100 \times \frac{1}{1+\prod_{i}\left(f_{i}^{e} / f_{i}^{\pi}\right)}
$$

where $f_{i}^{e}$ and $f_{i}^{\pi}$ are the fractions of electrons and pions, respectively, for which the quantity $i$ (one of the discrimination variables mentioned above) lies in a particular range. These fractions are determined from a separate study, detailed in Ref. [30]. In brief, samples of electrons from photoconversion pairs, and pions from $K_{s}^{0}$ decays are first divided into six momentum ranges. Then, the fractions of electrons and pions for each momentum range are calculated in narrow ranges of the discrimination 
variables listed above. It has been found that the algorithm EMPROB provides good discrimination between electrons and pions. About $75 \%$ of true electrons have $E M P R O B>80$, for all momentum ranges, while only about (1-2)\% of the pions do.

\subsection{Data Acquisition}

Data was collected by the E791 experiment from July 1991 to January 1992. Approximately each minute, the Fermilab Tevatron provided a $\pi^{-}$beam during a 23 -second "spill" period followed by a 34-second "interspill" while the Main Ring refilled and re-accelerated protons. The Data Acquisition (DAQ) system was tuned such that data were continually buffered, processed and written out to 42 Exabyte tape drives with no loss of productivity during the "interspill" period [27]. Figure 2.4 shows a schematic of the DAQ system. E791 data, read by the detector electronics and digitized by various front-end systems, was collected in parallel by eight Event FIFO Buffers (EFB's), then distributed through Event Buffer Interfaces (EBI's) to CPU processors in six VME crates, again running in parallel, where the data segments from individual detector systems were combined into formatted events. Finally the events were recorded on 42 8-mm magnetic tapes (seven tape drives connected to each of the six crates). The FIFO Buffers stored 80 Megabytes of data each, ensuring that the other DAQ components could run smoothly without any dead time. When the EFB's were detected to be nearly full, a signal inhibited the trigger. Six VME crates contained the CPU processors. Each was equipped with eight EBI's, each of which had access to one of the eight EFB's. The EBI's read the data out of the EFB's and passed it along to the CPU's. The EBI's maintained the control mechanism such that only one of the VME crates was used to read an EFB at any one time. Two Magnetic Tape Controllers (MTC's) were installed in each VME crate. The MTC's wrote data to seven tape drives at the same rate. A human operator was responsible for loading tapes into the drives and unloading them once filled. Tapes filled up in approximately three hours of beam time.

Three major software programs controlled the performance of the DAQ system. At the top level the VAX program provided a user interface, monitored the status 


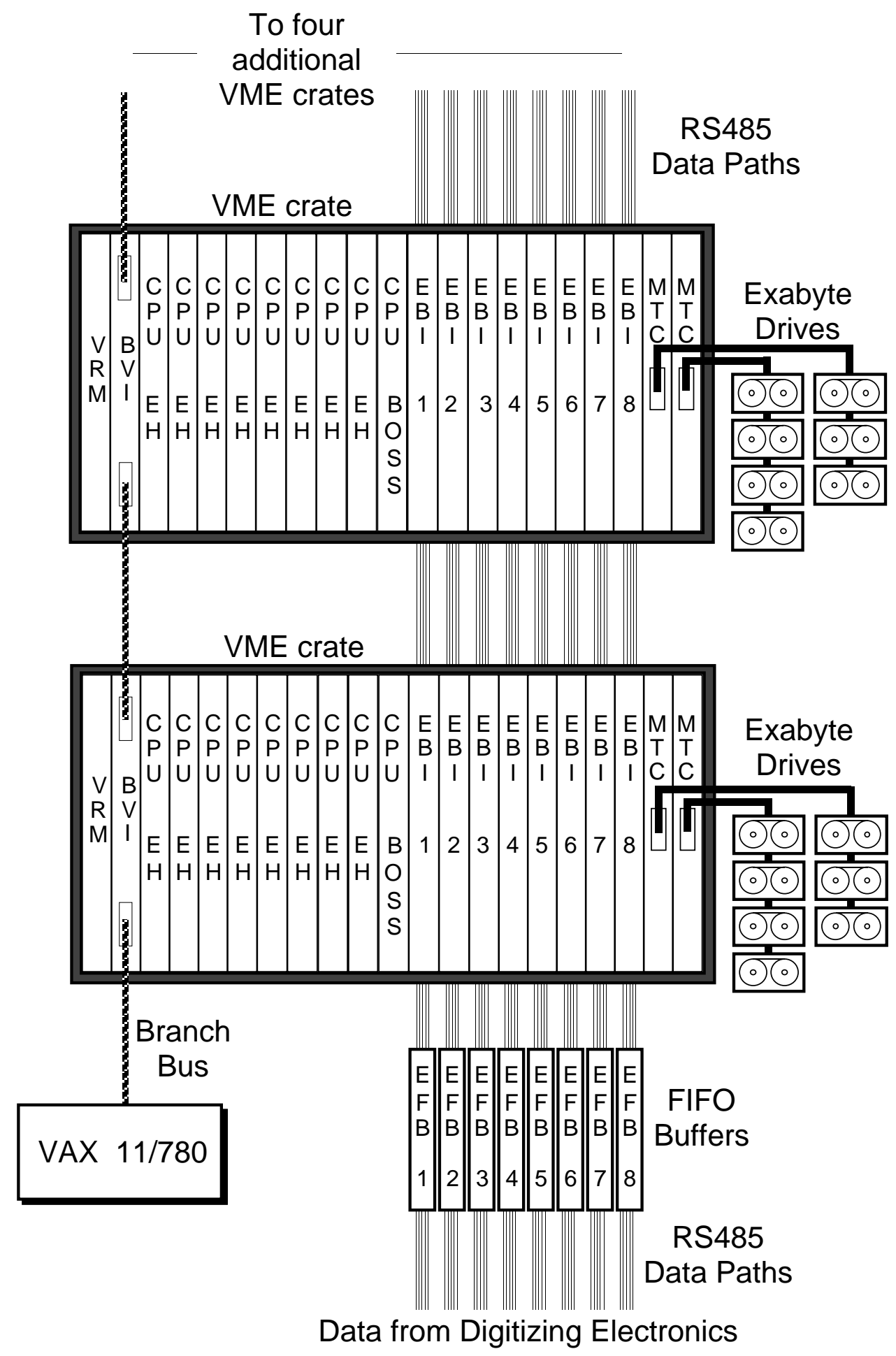

Figure 2.4: Schematic of the major components of the Data Acquisition system. 
of all the systems, and handled the run Start-up and End processes. The "Boss" program ran in one of the CPU's in each of the VME crates. It regulated the status of the other CPU's in the crate, determining whether they were in the event-collecting "grabber" or event-processing "muncher" mode. It also managed the writing of data to tape, selecting which MTC to use. The Event Handler (EH) program ran in two modes: "grabber" and "muncher". One of the nine CPU's in the VME crate acted as the "grabber" which read in event segments and placed them into its large event array. Once the "Boss" detected that the array was full, the EH was switched to the "muncher" mode, and another CPU switched to a "grabber". "Munching" involved formatting and compressing the data and grouping events into blocks for output to tape. "Muncher" mode was more time-consuming than "grabber", thus at any one time, only one CPU was "grabbing", while the others were busy "munching".

Overall the DAQ system performed well. The maximum data rate to tape was 9.6 Megabytes/s. The system encountered few catastrophic errors that resulted in complete shutdown, and recovery in each case was swift. In the nine-month period of data-taking, approximately $20 \times 10^{9}$ events were recorded on $24 \times 10^{3}$ tapes, which corresponds to 50 Terabytes of data. 


\section{Chapter 3}

\section{Data Selection}

For the form factor analysis, which is based on $100 \%$ of the E791 data sample, we study the decay chain $D^{+} \rightarrow \bar{K}^{* 0} e^{+} \nu_{e}, \bar{K}^{* 0} \rightarrow K^{-} \pi^{+}$(charge conjugate decay is implied). To select a clean signal, the data passes through numerous stages of selection criteria. Since the neutrino in the decay cannot be detected, we look for charm decays that have a three-prong decay (secondary) vertex that is well separated from the production (primary) vertex. The diagram in Figure 3.1 shows a typical

$D^{+} \rightarrow \bar{K}^{* 0} e^{+} \nu_{e}$ event where a beam track interacts with a target nucleon and a $D^{+}$ is produced (represented by the dotted line in the diagram). It then decays, and three charged tracks are reconstructed as having originated at the secondary vertex. Čerenkov information is used for hadron identification as described in Sec. 2.4. The electron probability algorithm, EMPROB, described in Sec. 2.5, is used for electron identification. The final stage of cuts uses a binary decision tree algorithm. The details of each level of selection are described in this chapter.

\subsection{Filter and Strip}

After the first stage of data reconstruction, loose cuts are applied to data. The beam track and a primary vertex must be found for each event. An event must also have at least one secondary vertex that is significantly separated from the primary. The significance of separation along the beam direction (SDZ) must be at least 4.0 for a 


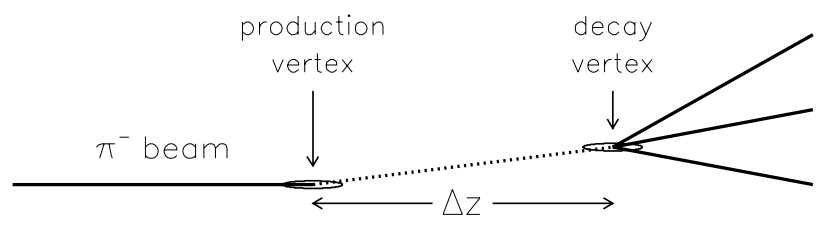

Figure 3.1: Diagram of a typical E791 event of interest to this analysis. The ovals represent the resolution on the vertices. Additional tracks originating at the production vertex are not shown.

secondary vertex with three or more tracks associated with it. SDZ is defined as

$$
\mathrm{SDZ}=\frac{\Delta z}{\sqrt{\sigma_{p r i}^{2}+\sigma_{s e c}^{2}}}
$$

where $\Delta z$ is the distance between the vertices along the beam $(z)$ direction, as shown in Figure 3.1, and $\sigma_{i}$ is the error on the $z$-position of the vertex $i$.

The next level of data selection is the strip. Further cuts are applied to winnow possible $D^{+} \rightarrow \bar{K}^{* 0} e^{+} \nu_{e}$ signal from the background dross. An event that passes this level of data selection must have a decent electron candidate (EMPROB $>70$ ) among the tracks in the secondary vertex. The SDZ cut is tightened to 5.0. In addition, each track in the vertex must pass several loose checks, such as (a) the $\chi^{2}$ for track fit is $<6.5$, and (b) the track is classified as at least Category 3 .

\subsection{Substrip and Microstrip}

Next level of data processing (substrip) took place at Kansas State University computing farms. Events passing these cuts are further stripped (microstrip) to pull off only those events that have a three-prong secondary vertex containing a lepton. The selection criteria for the combination of the substrip and microstrip are: 
1. Decent electron candidate in the vertex $($ EMPROB $>70)$.

2. The primary vertex lies upstream of the interaction counter $\left(z_{\text {prim }}<-1.0 \mathrm{~cm}\right)$.

3. The vector sum of the momenta of the three tracks in the secondary vertex, perpendicular to the flight direction of the $D^{+}\left(p_{T}\right.$ balance), is less than 1.5 $\mathrm{GeV}$.

4. Low probability for the tracks in the secondary vertex to have come from the primary vertex $\left(\chi^{2}\right.$ of impact parameter $\left.>6\right)$.

\subsection{Nanostrip}

The next level of cuts, the nanostrip, produces an event reduction of approximately 5 to 1 . The following cuts are implemented in the selection of the $D^{+} \rightarrow \bar{K}^{* 0} e^{+} \nu_{e}$ candidates:

1. Secondary Vertex Cuts

- Well separated (SDZ $>15)$ secondary vertex with 3 tracks, and charge \pm 1 .

- Vertex $\chi^{2} /$ dof less than 9.0.

- Vertex outside of a target foil by at least one standard deviation.

- Vertex lies upstream of the interaction counter $\left(z_{\text {sec }}<-0.4 \mathrm{~cm}\right)$.

2. Track Quality cuts

- All three tracks must be classified as Category 3, 7, or 15. Possible Category 3 "ghost" tracks are removed with a neural-net algorithm [31].

- The track $\chi^{2} /$ dof is less than 5.0.

3. Particle Identification cuts

- EMPROB greater than 80 for one of the tracks. This track is identified as an electron. 
- The charge on the track identified as the electron must equal the charge of the secondary vertex.

- One track with kaon probability, as determined from the Čerenkov information, greater than 0.13 .

4. Mass cuts

- The invariant mass of the $K^{* 0}$ candidate, $M_{K \pi}$, must be between 0.75 and $1.05 \mathrm{GeV}$.

- The three-prong candidate is not consistent with being a misidentified $D^{+} \rightarrow K^{-} \pi^{+} \pi^{+}$decay. The invariant mass $M_{K \pi \pi}$, where the electron track is reconstructed as a pion, must lie outside the $M_{K \pi \pi}$ window, 1.82 to $1.92 \mathrm{GeV}$.

- The minimum kinematically allowed mass of the $D^{ \pm}$candidate must be between 1.1 and $2.3 \mathrm{GeV}$. It is defined as

$$
M_{\text {min }}=\sqrt{m_{v i s}^{2}+p_{T}^{2}}+\sqrt{m_{\nu}^{2}+p_{T}^{2}}
$$

where $m_{v i s}^{2}$ and $p_{T}^{2}$ refer to the invariant mass and the sum of the transverse momenta, respectively, of the three visible particles. The $D^{ \pm}$candidate mass cannot be determined exactly due to the missing neutrino. At best, the neutrino momentum can be reconstructed up to a quadratic ambiguity (See Sec. 4.4). The $M_{\text {min }}$ calculation neglects the longitudinal component of the neutrino momentum.

\subsection{Analysis Cut Selection}

The final set of cuts produces a very clean $D^{+} \rightarrow \bar{K}^{* 0} e^{+} \nu_{e}$ sample. Cuts on the three following parameters are made.

1. Kaon probability of the candidate kaon $>0.4$.

2. Pion probability of the pion candidate $>0.4$. 
3. Probability that the electron track comes from photoconversion: PPSQ > $0.0002 \mathrm{GeV}^{2}$. PPSQ is related to the invariant mass of the photoconversion pair. Defined formally it is

$$
\operatorname{PPSQ}=\left|p_{T, 1}\right|^{2}+\left|p_{T, 2}\right|^{2}
$$

where $p_{T, 1}$ and $p_{T, 2}$ are the transverse components of the two track momenta with respect to the total momentum vector. The electron candidate track is paired with every other track in the event and the minimum PPSQ is found. In the electron identification study [30] it was found that electrons coming from photoconversions peak at PPSQ $<4 \times 10^{-5} \mathrm{GeV}^{2}$. PPSQ distribution for other electrons is flat. Thus by cutting at PPSQ $>0.0002 \mathrm{GeV}^{2}$ all photo conversion electrons are effectively removed from the data sample.

In addition, the Binary Decision Tree Algorithm commercial software CART [32] is used to determine the set of splits in a multidimensional parameter space that best separates signal from background. The program needs to be "trained" on a small sample of signal and background events.

CART was trained on a small subset of the data. Right-sign events in which the $K^{-}$and $e^{+}$have opposite charge, as would be expected for $D^{+} \rightarrow K^{-} \pi^{+} e^{+} \nu_{e}$ (and charge conjugate events) in the $K^{* 0}$ mass window, $0.85<M_{K \pi}<0.94 \mathrm{GeV}$, that also have $M_{\min }$ lying between 1.6 and $2.0 \mathrm{GeV}$, are designated as "signal". Likewise, wrong-sign events in which $D^{+} \rightarrow K^{+} \pi^{-} e^{+} \nu_{e}$ (and charge conjugate candidates) within the same $M_{K \pi}$ and $M_{\min }$ windows are designated as "background". Because many of the so-called "signal" events at this stage were in fact background, it was necessary to weight events in such a way that it would be more costly for CART to misidentify a "background" event as "signal", than vice versa. After some tuning, a $3 / 1$ cost of misidentification of "background" / "signal" was chosen, as it produced the best separation of true signal from background. CART incorporates these costs at every stage of tree building.

The parameters that were chosen for the discrimination between signal and background are described here. In these descriptions, "standard deviation" refers to the 
measured uncertainty for the relevant quantity.

1. Sigma - Number of standard deviations the secondary vertex is outside the target foil or interaction counter.

2. SDIP - The distance-of-closest-approach in the $x-y$ plane of the reconstructed $D^{+}$candidate (allowing for the missing neutrino information) with respect to the primary vertex, measured in standard deviations. We account for the missing neutrino information by calculating the maximum possible "miss distance" $d_{\text {max }}$ corresponding to the assumptions that the neutrino momentum $\mathbf{p}_{\nu}$ is perpendicular to the vector sum of the momenta of the three visible particles $\mathbf{p}_{\mathbf{v i s}}$ and that the tracks are $D^{+}$decay products. Then we have

$$
m_{D}^{2}=\left(p_{v i s}+p_{\nu}\right)^{2}=m_{v i s}^{2}+2 E_{v i s} E_{\nu}+m_{\nu}^{2}
$$

where $m_{v i s}, p_{v i s}$, and $E_{v i s}$ are the invariant mass, momentum, and energy of the visible particles. Since the neutrino is massless, $E_{\nu}=\left|\mathbf{p}_{\nu}\right|$. Then Eq. 3.3 reduces to

$$
\left|\mathbf{p}_{\nu}\right|=\frac{m_{D}^{2}-m_{v i s}^{2}}{2 E_{v i s}} .
$$

Therefore $d_{\max }$ is given by

$$
d_{\text {max }}=\frac{\left|\mathbf{p}_{\nu}\right|}{\left|\mathbf{p}_{\nu}+\mathbf{p}_{\text {vis }}\right|} \times r
$$

where $r$ is the distance between the primary and secondary vertices. The parameter SDIP is then given by

$$
\mathrm{SDIP}=\frac{\left|d i p-d_{\max }\right|}{\sigma_{\text {dip }}}
$$

where dip and $\sigma_{d i p}$ are the distance-of-closest-approach of the vector sum of the momenta of the three visible tracks to the primary vertex and the error on this measurement, respectively.

3. $\chi_{v t x}^{2}-$ The $\chi^{2}$ of the secondary vertex fit. 
4. SDZ - The separation of primary and secondary vertices measured in standard deviations.

5. $\tau_{\text {decay }}$ - Decay lifetime of the $D^{+}$candidate calculated using the calculated neutrino momentum (see Sec. 4.4).

6. SPISO - The minimum distance-of-closest-approach of the three daughter tracks to the primary vertex measured in standard deviations.

7. Sratio - The product of ratios of SSISO/SPISO for each of the three tracks. SSISO is the distance-of-closest-approach of a track to the secondary vertex.

The small training samples were constructed from approximately $15 \%$ of the data. Three sets of five mutually exclusive training samples were used. Each of the fifteen samples was used to create a decision tree. The resulting sets of cuts were applied to the data and Monte Carlo, and a standard fit was performed. In the end, the set of cuts with results for the form factor ratios nearest to the average values was chosen. The resulting tree has two nodes, of which one is considered "signal" and the other "background". One cut on a linear combination of the input parameters was chosen:

$$
\begin{aligned}
& \left(2.79 \times 10^{-4}\right) \mathrm{Sigma}-\left(2.39 \times 10^{-4}\right) \mathrm{SDIP}+ \\
& \left(2.39 \times 10^{-5}\right) \mathrm{SDZ}-\text { Sratio }-1.37 \times 10^{-3}>0
\end{aligned}
$$

The left-hand side of the above equation is plotted in Figure 3.2. The parameters most useful in signal/background discrimination, as determined by CART, were Sigma and SDIP.

\section{5 $D^{+} \rightarrow \bar{K}^{* 0} e^{+} \nu_{e}$ Signal}

After all the selection criteria (including the CART cut) have been applied, there are 3595 right-sign and 602 wrong-sign events in the signal region $\left(0.85<M_{K \pi}<0.94\right.$ $\left.\mathrm{GeV} ; 1.6<M_{\min }<2.0 \mathrm{GeV}\right)$ in the final data sample. The top left plot in Figure 3.3 shows the $M_{\min }$ distribution for both the right-sign and wrong-sign events following all 


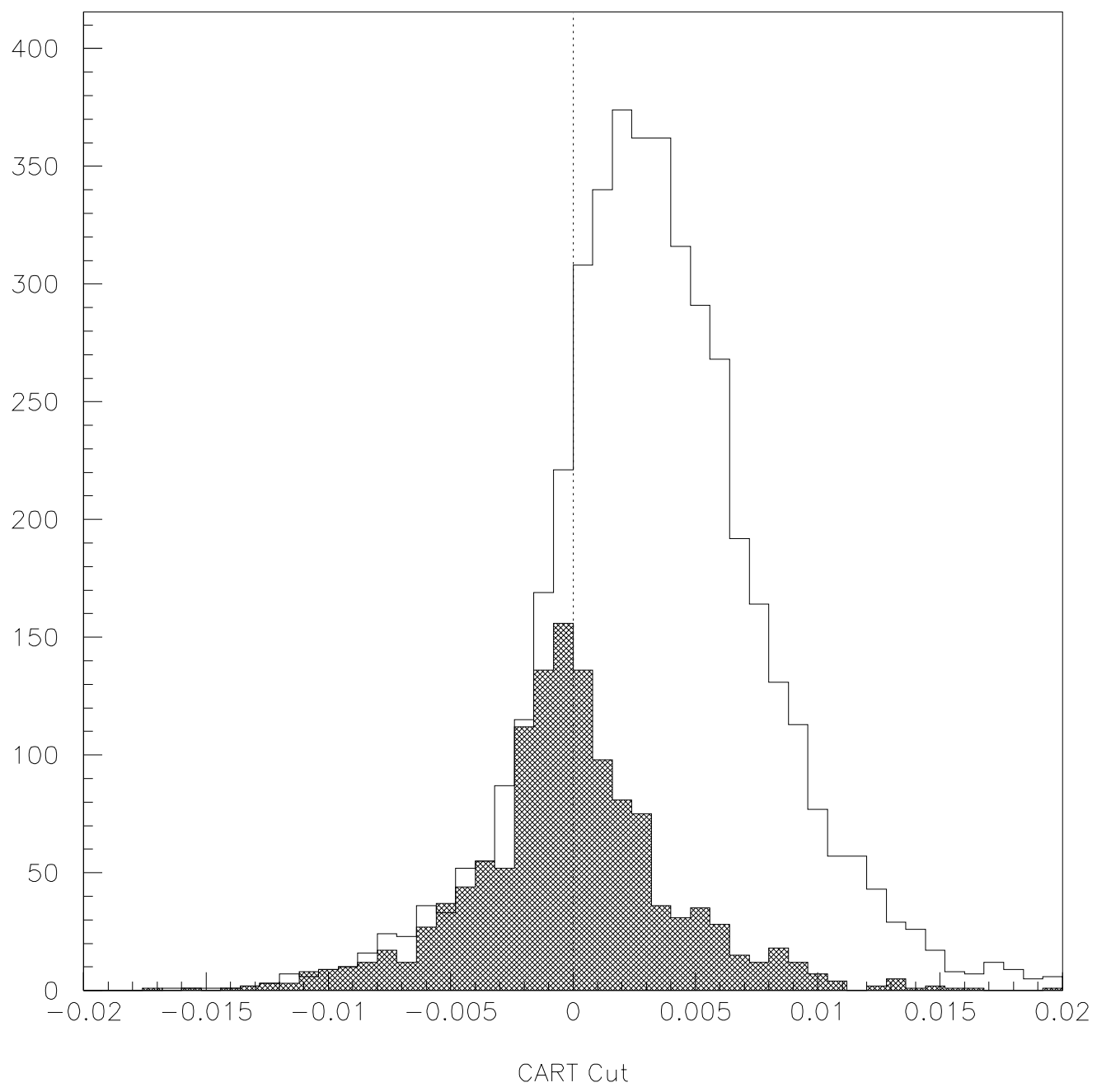

Figure 3.2: Right-sign (clear histogram) and wrong-sign (shaded) distribution of the linear combination chosen by CART as summarized in Eq. 3.7. Mass and particle identification cuts have already been applied. Events lying to the right of the dashed line are kept, and the rest are discarded. 
cuts, excluding the $M_{K \pi}$ cut. The arrows indicate the $M_{\min }$ range $\left(1.6<M_{\min }<2.0\right.$ $\mathrm{GeV}$ ) used in the fit. The $M_{K \pi}$ distribution is shown in the bottom left plot of Figure 3.3 for those events lying in the $1.6<M_{\min }<2.0 \mathrm{GeV}$ window. The right plot shows the difference between the right-sign and wrong-sign distributions fitted to a Breit-Wigner function, where the central mass and width values are fixed to be the mass and width of the $\bar{K}^{* 0}$. The arrows indicate the $M_{K \pi}$ range $\left(0.85<M_{K \pi}<0.94\right.$ $\mathrm{GeV}$ ) of the signal region. 

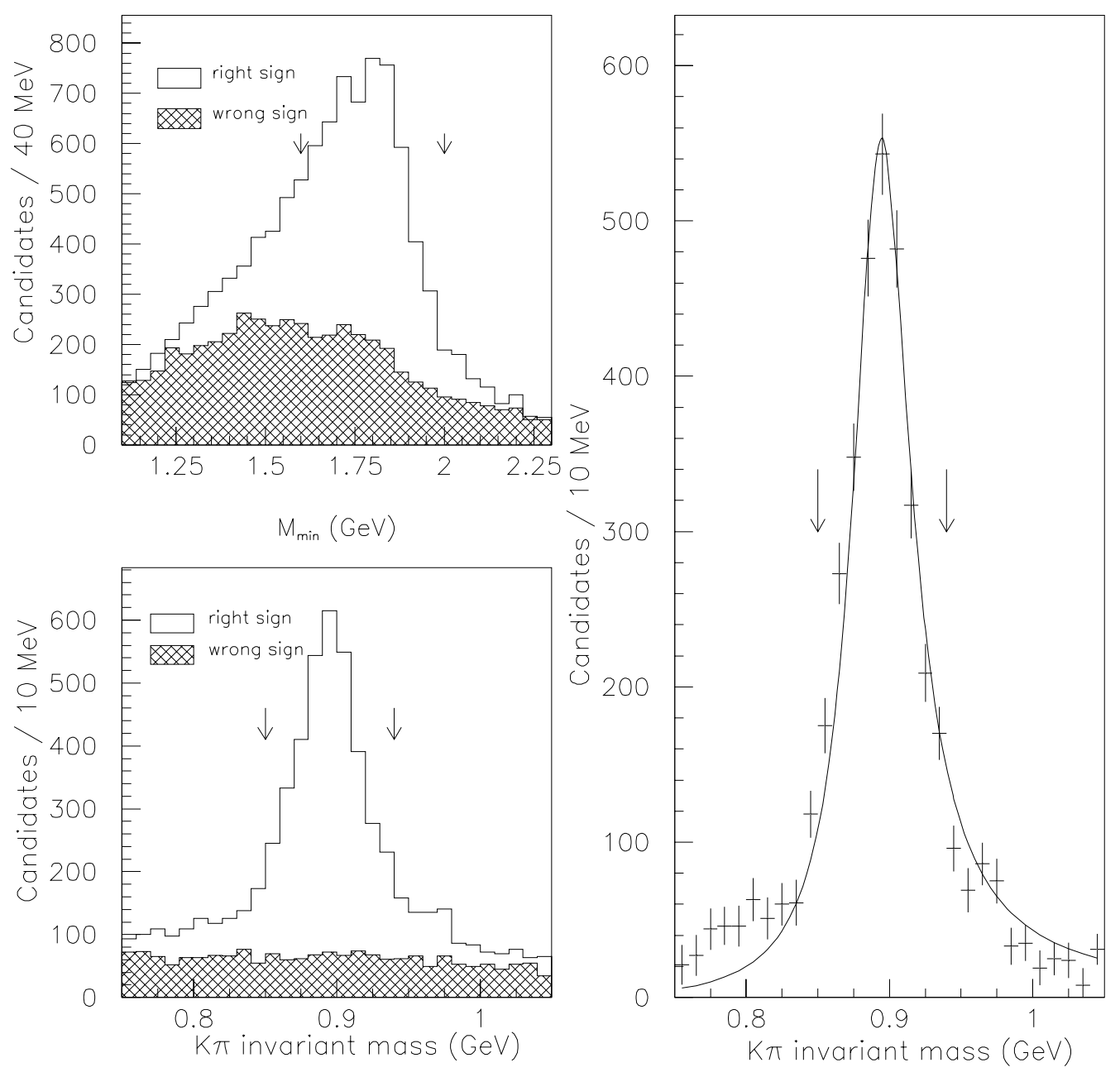

Figure 3.3: Mass distributions for $D^{+} \rightarrow \bar{K}^{* 0} e^{+} \nu_{e}$ candidate events in the full E791 data sample. The distributions are described in the text. 


\section{Chapter 4}

\section{Fitting Technique}

\subsection{Maximum Likelihood Method}

The fitting procedure used in this analysis was developed by E691 for a similar analysis [33]. An unbinned maximum likelihood fit is performed with the CERN functionminimization program MINUIT [34]. A typical likelihood function $\mathcal{L}$ is the product of probabilities $P_{i}$ for each data event used in the fit. Each $P_{i}$ depends on the set of kinematic variables $\mathbf{x}$ associated with the data point, and the parameters of the fit, $\mu$, which in our case are the ratios of form factors, $r_{2}$ and $r_{V}$ :

$$
\mathcal{L}=\prod_{i=1}^{n} P_{i}(\mathbf{x} ; \mu)
$$

The value of $\mu$ which maximizes $\mathcal{L}$ gives the best estimate for $\mu$. Since the evaluation of $\mathcal{L}$ involves taking a product of many large and small numbers, we minimize the negative $\log$ of the likelihood function instead. $P_{i}$ is given by the value of the differential decay rate $G(\mathbf{x} ; \mu) \equiv \frac{d \Gamma}{d \mathbf{x}}$, defined in Chapter 1, Equation 1.7, calculated at the point $\mathbf{x}$ for a given set of fit parameters $\mu$, normalized by the integral of $G(\mathbf{x} ; \mu)$ over the entire kinematic variable space:

$$
P_{i}(\mathbf{x} ; \mu)=\frac{G(\mathbf{x} ; \mu)}{\int G(\mathbf{x} ; \mu) d \mathbf{x}} .
$$


This method is standard for finding the best estimate for the parameters $\mu$ given the probability distribution $G(\mathbf{x} ; \mu)$. However it cannot account for the effects of acceptance and reconstruction of the data. The true physical values of the kinematic variables are indeed distributed according to $G(\mathbf{x} ; \mu)$, but we only measure the kinematic variables of the events that have been subjected to the smearing and inefficiencies of the reconstruction procedure. It is therefore necessary to relate the reconstructed distribution of the kinematic variables to the true one, in order to extract the values of the form factor ratios using the maximum-likelihood-fit method described above.

\subsection{Acceptance and Reconstruction Effects}

One way of incorporating the effects of acceptance and smearing is to compare the distribution of data events to a large set of Monte Carlo events that have been affected by the same reconstruction and acceptance effects. The point of using Monte Carlo is that the events are generated with known form factors, and the true distributions of the kinematic variables is known. For each Monte Carlo event $j$ we have both the set of true values of the kinematic variables, $y_{j}$, and the set of reconstructed values, $\tilde{y}_{j}$. We can then compare the distribution of the kinematic variables in the data to the reconstructed distribution in the Monte Carlo, and get a handle on the true data distribution, and hence the values of the form factors.

We do this by counting, and weighting appropriately, the number of reconstructed Monte Carlo points $\tilde{y}_{j}$ that lie within a small five-dimensional volume $V_{i}$ surrounding each data point ${ }^{1} x_{i}$ in the kinematic variable space. The likelihood probability for each data event then becomes a sum of the weights $W\left(y_{j} ; \mu\right)$ (described below) of all the $\tilde{y}_{j}$. Equation 4.2 now becomes:

$$
P_{i}=\frac{\sum_{\tilde{y}_{j} i n V_{i}} W\left(y_{j} ; \mu\right)}{\sum_{j=1}^{m} W\left(y_{j} ; \mu\right) V_{i}}
$$

\footnotetext{
${ }^{1}$ For data events near the edge of the kinematic variable space, the volume size $V_{i}$ is reduced such that it lies entirely in the physically allowed region, and is centered on the data point.
} 
where the summation in the denominator is the sum of the weights for all $m$ Monte Carlo events. The Monte Carlo events are generated with one set of form factors, $\mu_{\mathbf{0}}$. Therefore the weight $W\left(y_{j} ; \mu\right)$ is the ratio of the differential decay rate $G(\mathbf{x} ; \mu)$ for some trial value of the form factors, $\mu$, to that for the generated values $\mu_{0}$ :

$$
W\left(y_{j} ; \mu\right)=\frac{G(\mathbf{x} ; \mu)}{G\left(\mathbf{x} ; \mu_{0}\right)} .
$$

Hence for the generated form factors $\mu_{\mathbf{0}}$, all the weights are equal to one, and the sum of the weights becomes just the number of Monte Carlo events in $V_{i}$.

\subsection{Background}

The final aspect of the fitting procedure is the inclusion of the background estimate in the fit. Although the data selection procedure produces a fairly clean sample of $D^{+} \rightarrow \bar{K}^{* 0} e^{+} \nu_{e}$ right-sign events, there is still a substantial number of background events that fall under the signal peak, as shown in the bottom left plot of Figure 3.3. The background may be due to misreconstructed charm events, or just random combinations of tracks that happen to pass all the selection criteria. We assume that the background in right-sign data is modeled by the distribution of wrong-sign data. We introduce the background information into the fit in a similar manner as the Monte Carlo. As with the treatment of the Monte Carlo in the preceding section, we count the weights of all the wrong-sign events that lie within a small five-dimensional volume $V_{i}^{B}$, and normalize by the sum of the weights over all the background events. Under the assumption that the right-sign background is distributed exactly like the wrong-sign events, all the weights are then equal to one. The final expression for the likelihood function used by the fit is then

$$
\mathcal{L}=\prod_{i=1}^{n}\left[\left(n-n_{B}\right) \frac{\sum_{\tilde{y}_{j} \text { in } V_{i}} W\left(y_{j} ; \mu\right)}{\sum_{j=1}^{m} W\left(y_{j} ; \mu\right) V_{i}}+\frac{n_{i}}{V_{i}^{B}}\right]
$$

where $n$ is the total number of events in the right-sign sample, $n_{B}$ is the total number of expected background events in the right-sign sample, and $n_{i}$ is the number of 
wrong-sign events lying in $V_{i}^{B}$.

This powerful fitting technique is quite general, allowing a fit in any number of dimensions, and modeling the effects of acceptance and reconstruction, as well as the distribution of the background events. There are a few disadvantages to this procedure. The main one is the necessity for a very large Monte Carlo sample, such that every region of the five-dimensional kinematic variable space is well represented. In the limit of finite size of the Monte Carlo sample, any dependence of the fit on arbitrary volume sizes, $V_{i}$ and $V_{i}^{B}$, contributes to the systematic uncertainty on the resulting form factors. Chapter 7 discusses the systematic checks that have been performed on the fitting technique.

\subsection{Neutrino Momentum}

In order to calculate the kinematic variables used by the fitting technique we need to compute the momentum of the neutrino. Since it is a neutral particle, and interacts only through the weak interaction, it leaves no trace in the detector. Therefore we must deduce the neutrino momentum from the reconstructed momenta of the visible particles. The component of the neutrino momentum transverse to the $D$ line of flight, $p_{T}$ is equal and opposite to the sum of the transverse momenta of the three visible particles. The direction of the $D$ is estimated from the positions of the primary and secondary vertices. The longitudinal component of the neutrino momentum is calculated with the constraint on the mass of the parent particle to be equal to $M_{D}$ in the reference frame where the component of the visible momentum along the direction of the $D$ is zero. It is given by

$$
p_{\text {long }}^{\nu}= \pm\left[\left(\frac{M_{D}^{2}-m_{v i s}^{2}-2 p_{T}^{2}}{2 E_{\text {vis }}}\right)^{2}-p_{T}^{2}\right]^{1 / 2}
$$

where $m_{v i s}$ and $E_{v i s}$ are the invariant mass and the total energy of the visible particles. The term inside the parentheses is the total neutrino momentum $p_{\nu}$. We use the negative solution for $p_{l o n g}^{\nu}$. A negative solution was also used in the form-factor 
analyses of E653 [16] and E691 [15]. A Monte Carlo study revealed that the negative solution corresponded to the correct value for the neutrino momentum approximately $48 \%$ of the time. For cases in which we get an unphysical result for $p_{l o n g}^{\nu}$, i.e., $p_{\nu}<p_{T}$, we set $p_{\text {long }}^{\nu}=0$ and $p_{\nu}=p_{T}$. 


\section{Chapter 5}

\section{Monte Carlo Simulation}

Hadronic interactions in the E791 Monte Carlo are generated using the CERN software package PYTHIA 5.7 [35]. The package JETSET 7.4 [36] is then used to simulate jet fragmentation and particle decays. The simulated charm decays are then passed through a simulation of the E791 spectrometer, where the interactions of the "particles" with the various detector systems are recorded in a manner similar to real data. Approximately 20 million $c \bar{c}$ Monte Carlo events, with at least one $D^{+} \rightarrow \bar{K}^{* 0} e^{+} \nu_{e}$ (or charge conjugate mode) in the event, were generated on the Kansas State University compute cluster for this analysis. The Monte Carlo events were generated according to the differential decay rate described in Equations 1.7 and 1.8, with form factor ratios $r_{2}=0.82$ and $r_{V}=2.00$. These are the central values determined by E653. The projected distributions of the four kinematic variables in the Monte Carlo are plotted in Figure 5.1. These are the generated distributions that have not yet been affected by the reconstruction and data selection.

The Monte Carlo events were passed through the same chain of analysis cuts as data. Of the $\sim 20 \times 10^{6}$ generated $D^{ \pm}$events $\sim 73 \times 10^{3}$ pass all the selection criteria. The numbers of Monte Carlo events passing each stage of selection are summarized in Table 5.1. Three times as many $D^{-}$as $D^{+}$events were generated, by mistake. There was also a problem involving repeated starting seeds in the generation of the MC events which resulted in many events being duplicated. The duplicated events were removed from the final set based on the values of the four kinematic variables 

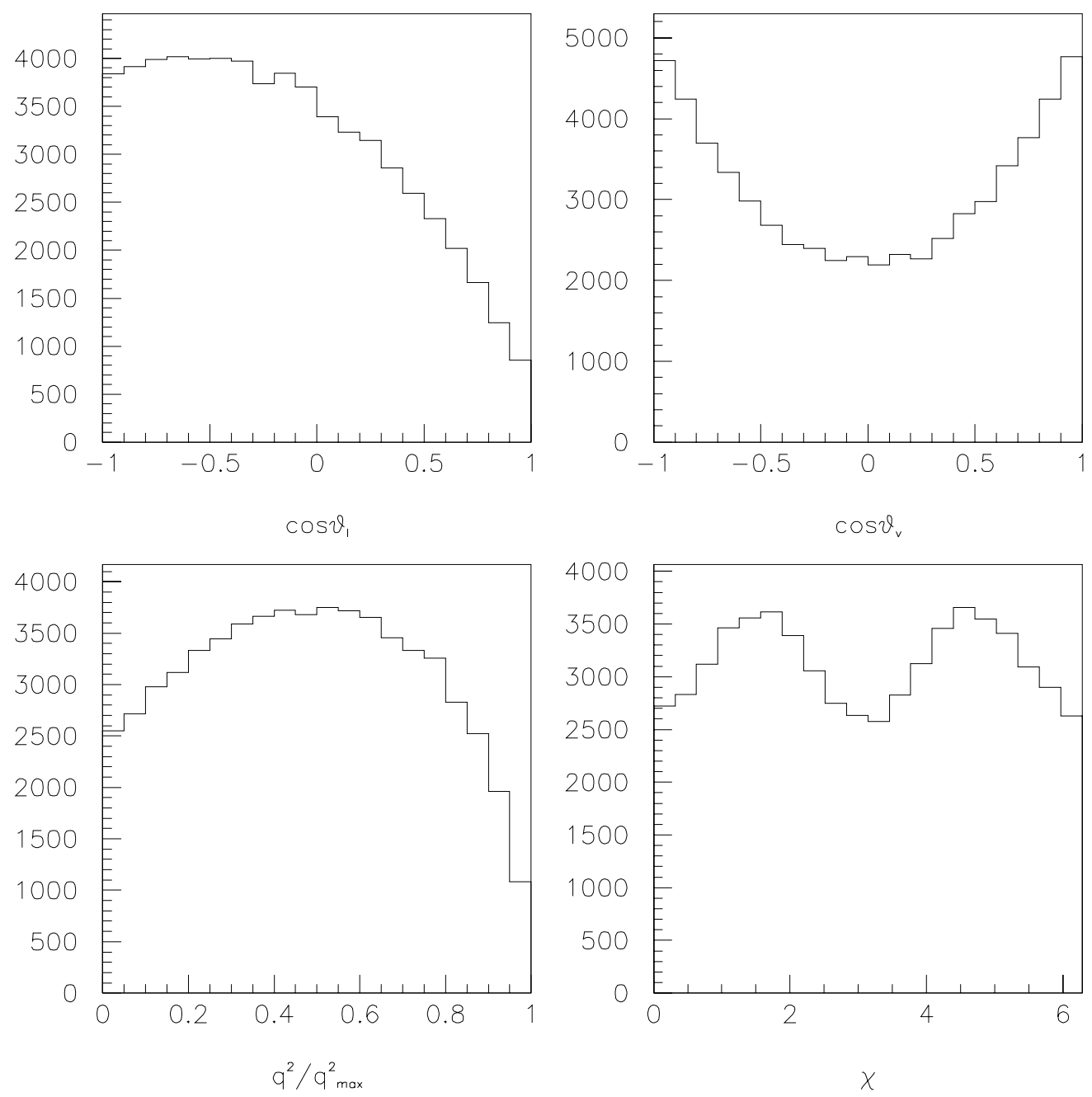

Figure 5.1: Distributions of the four kinematic variables as generated by the Monte Carlo. 


\begin{tabular}{|l|rc|}
\hline Analysis Stage & \# of Events & \% passing \\
\hline Generated (approx.) & 20000000 & 100.00 \\
\hline Filter & 9271560 & 46.36 \\
\hline Strip & 6996477 & 34.98 \\
\hline Sub-Strip & 2684314 & 13.42 \\
\hline Micro-Strip & 1292095 & 6.46 \\
\hline Nano-Strip & 408738 & 2.04 \\
\hline Analysis Cuts \& & 73161 & 0.37 \\
Duplicates Removed & & \\
\hline
\end{tabular}

Table 5.1: Number of Monte Carlo events passing each stage of cuts.

in the truth table matching those of a previously seen event exactly. Approximately $15 \%$ of the Monte Carlo events were found to be duplicates. Figure 5.1 excludes the duplicate events.

The effects of acceptance on the distributions of the kinematic variables can be seen in Figure 5.2. The acceptance as a function of the kinematic variables is plotted (in arbitrary units). The distributions for the variables $\chi, q^{2}$, and $\cos \theta_{V}$ are fairly flat, implying that no area of the phase space spanned by these three variables, is affected by the data selection disproportionately. The distribution for the other kinematic variable, $\cos \theta_{l}$, on the other hand, shows a significant depletion of events with $\cos \theta_{l}<0$; i.e., with leptons traveling toward the recoiling $D$ in the rest frame of the virtual $W$. This deficit may be caused by the electron identification criteria, which requires that the electron track intersect the electromagnetic calorimeter. Also, the lower cut on $M_{\min }$ rejects $D$ candidates with low lepton momentum, which correspond to events with small values of $\cos \theta_{l}$.

There are two possible sources of smearing resulting from the reconstruction and data selection procedure. The first is due to detector effects. The incorrect choice for the neutrino momentum solution is the second source of smearing of the kinematic variables. As mentioned before there is a quadratic ambiguity in the choice for the longitudinal component of the neutrino momentum (see Sec. 4.4). In this analysis we always choose the negative solution, which is not always the correct choice. Figures 5.3 

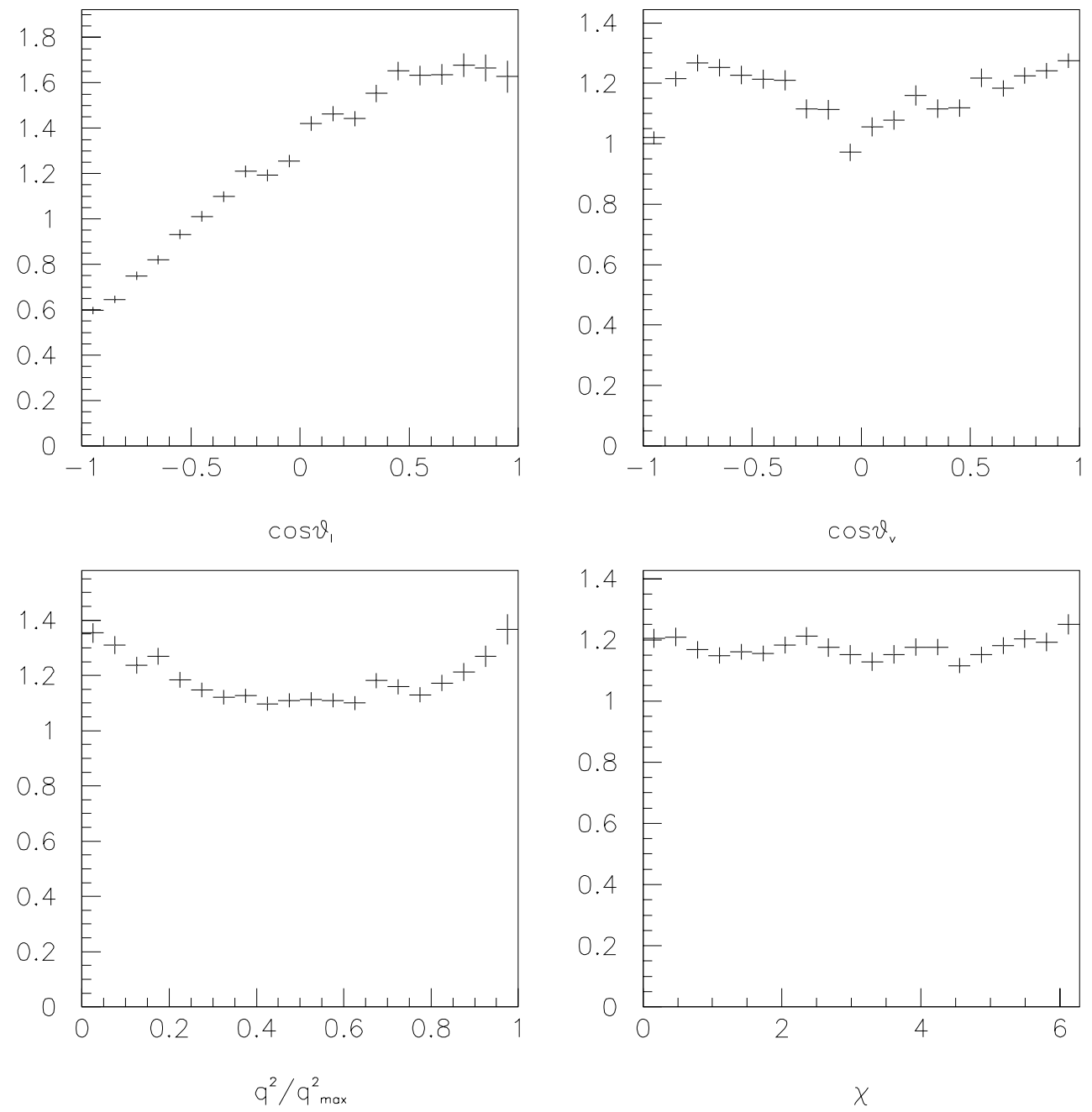

Figure 5.2: Efficiency of the reconstruction and data selection as a function of each of the four kinematic variables. The vertical scale is arbitrary. 
and 5.4 show the difference between the reconstructed value of a kinematic variable and the generated value of the variable for events passing all the data selection criteria, for each of the two possible choices for the neutrino momentum solution. The means of all distributions are very close to 0 , as expected. The RMS widths are also quite small, except in the case of the $\chi$ distribution, where the reconstructed $\chi$ may be as much as $\pi$ radians away from the true value. The shaded histograms show smearing when the effects due to the incorrect choice for the neutrino momentum solution are removed. It is evident that the poor resolution in $\chi$ is due to the incorrect choice for the neutrino momentum solution. There is little difference in the amount of smearing introduced when either the positive or negative solution for the neutrino momentum is chosen as can be seen in Figures 5.3 and 5.4.

Figure 5.5 shows a comparison of momentum distributions of D mesons in data and Monte Carlo. All reconstruction criteria have been applied. The top plot shows the magnitude of the total D momentum. The Monte Carlo distribution matches the data well. The bottom plot shows the magnitude of the component of the $\mathrm{D}$ momentum transverse to the $z$-axis. Here the agreement between Monte Carlo and data is quite poor. This may be due to a well-known problem of the degradation of the performance of the Drift Chamber over time (see Sec. 2.3.3) which is not well simulated by the Monte Carlo. The effect of this disparity in the two distributions is further discussed in Appendix B. 

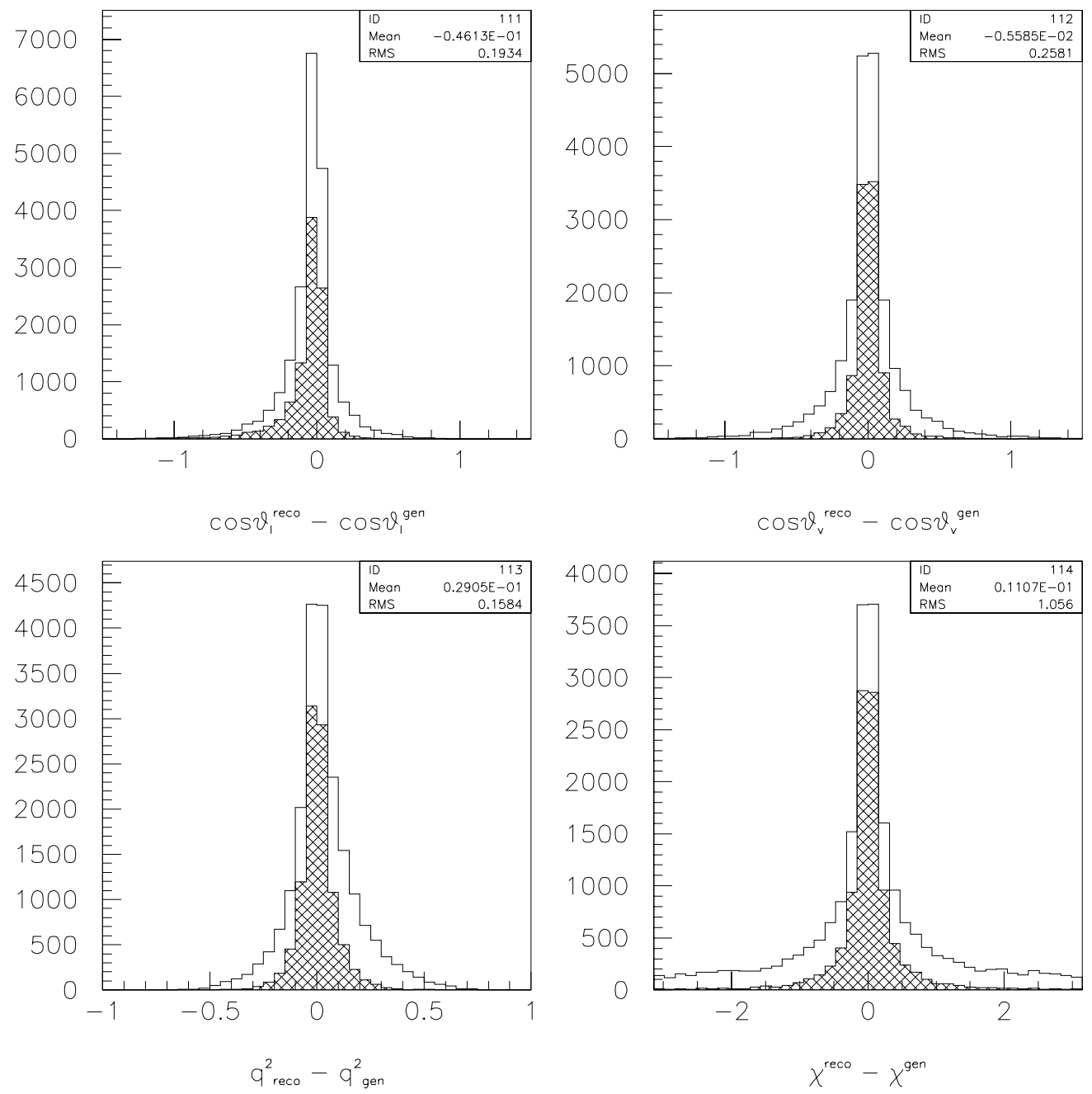

Figure 5.3: Difference between the generated and reconstructed values of the four kinematic variables in the Monte Carlo when the negative neutrino momentum solution is used. The shaded histogram represents the smearing due solely to detector effects. All selection criteria have been applied. 

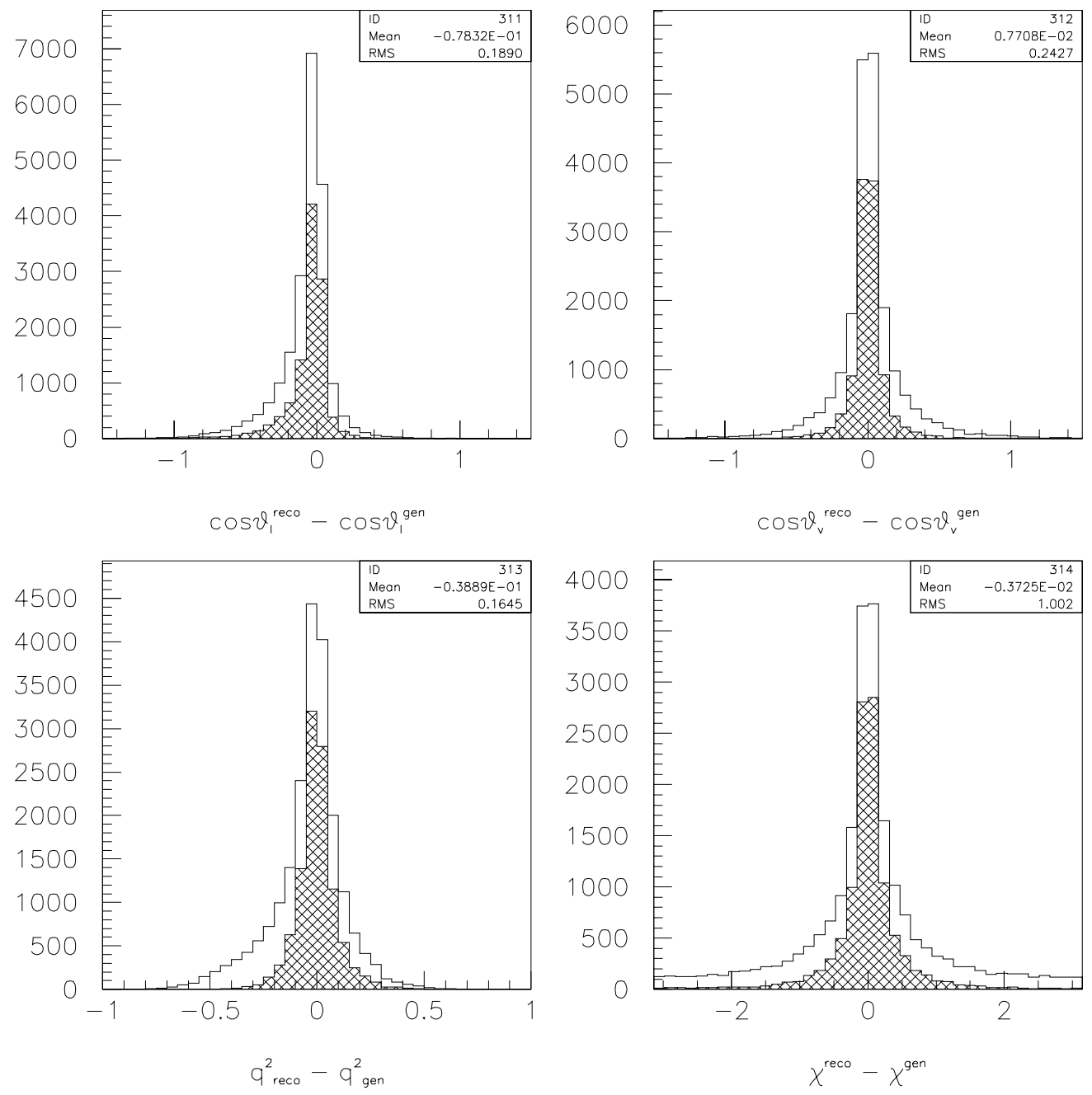

Figure 5.4: Difference between the generated and reconstructed values of the four kinematic variables in the Monte Carlo when the positive neutrino momentum solution is used. The shaded histogram represents the smearing due solely to detector effects. All selection criteria have been applied. 

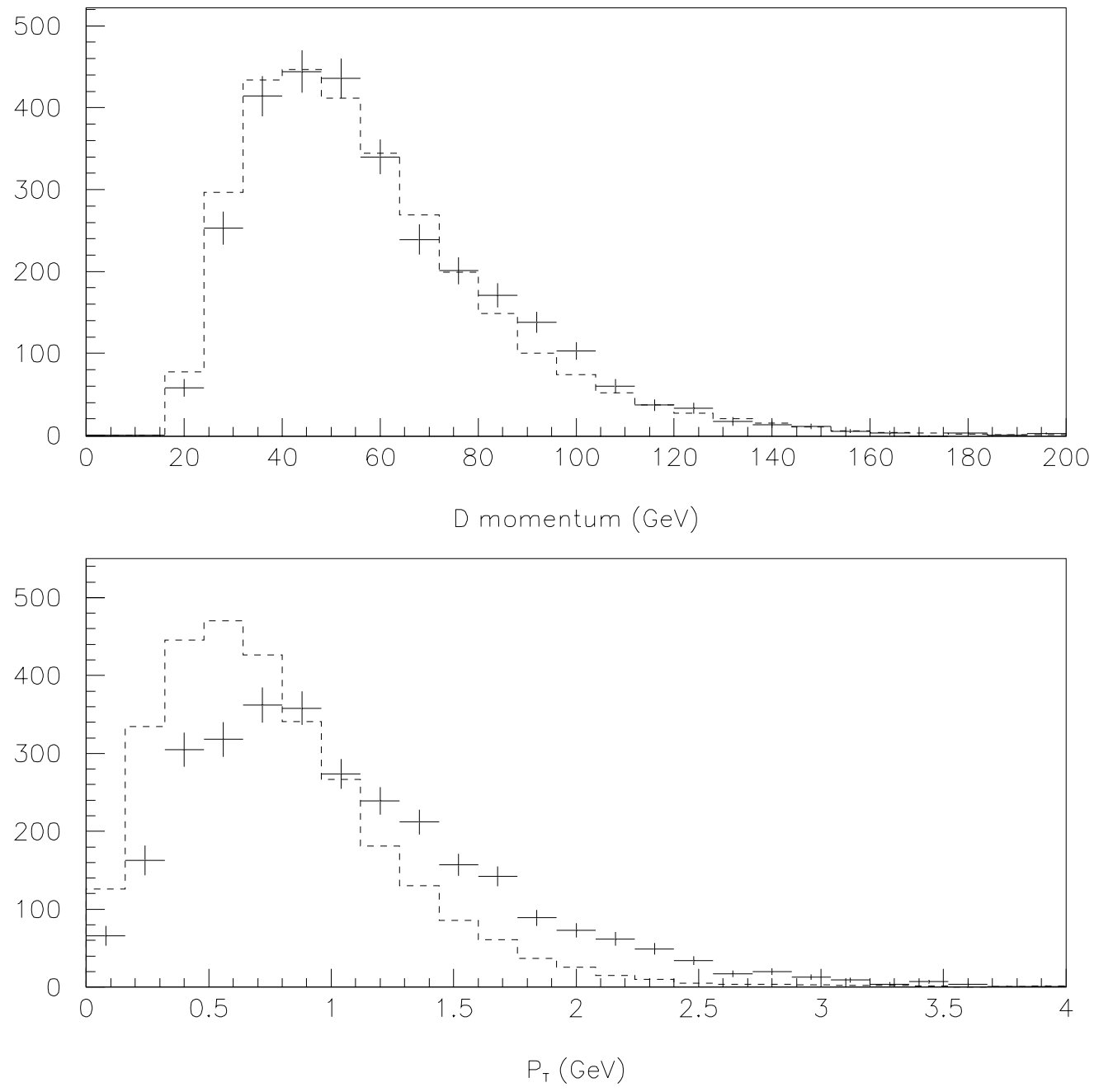

Figure 5.5: Momentum distributions for the $D$ meson in data (crosses) and Monte Carlo (histogram). Top plot shows the magnitude of the $D$ momentum. The bottom plot shows the magnitude of the momentum component transverse to the $z$-axis. All selection criteria have been applied. 


\section{Chapter 6}

\section{Particle Identification Efficiency}

In order to implement the fitting technique described in Chapter 3, it is necessary to accurately simulate the detector in the Monte Carlo. Studies by other members of the E791 collaboration [37] have shown that there are inconsistencies between the data and the Monte Carlo. This analysis depends heavily on the ability to properly identify the three tracks in the decay vertex. The following two sections describe studies made of particle identification efficiencies in the data and Monte Carlo and their effects on this analysis.

\subsection{Electron Identification}

A study was done to determine whether the efficiency of electron identification is the same in data and Monte Carlo. A sample of electrons from photo conversion pairs $\left(\gamma \rightarrow e^{+} e^{-}\right)$was used in the study. A track is considered an electron candidate if the transverse momentum of this track and another oppositely charged track that is very likely an electron $(\mathrm{EMPROB}>90)$ is very small compared to the total momentum of the two tracks, as is expected for $\gamma \rightarrow e^{+} e^{-}$. Approximately $1.4 \times 10^{6}$ data events and $2.25 \times 10^{6}$ Monte Carlo charm events were used in this study, producing $1.8 \times 10^{5}$ electron candidates each. Figure 6.1 shows the efficiency of electron identification as a function of the electron momentum for a specific cut on EMPROB $(>80)$ for both data and Monte Carlo in the top plot. Electron identification is overall less efficient 


\begin{tabular}{|c|c|}
\hline Momentum Range $(\mathrm{GeV})$ & Weight \\
\hline $0-3$ & $1.160 \pm 0.047$ \\
$3-6$ & $1.065 \pm 0.009$ \\
$6-9$ & $0.995 \pm 0.011$ \\
$9-12$ & $1.004 \pm 0.021$ \\
$12-15$ & $1.016 \pm 0.031$ \\
$15-18$ & $1.028 \pm 0.041$ \\
$18-21$ & $1.086 \pm 0.058$ \\
$21-24$ & $1.097 \pm 0.067$ \\
$24-27$ & $1.096 \pm 0.091$ \\
$>27$ & $1.137 \pm 0.114$ \\
\hline
\end{tabular}

Table 6.1: Weights used to correct the inaccuracies in the Monte Carlo electron identification efficiency for different momentum regions. EMPROB cut is 80 .

in the Monte Carlo than in data probably due to the fact that fewer tracks appear to be associated with electromagnetic calorimeter energy in the Monte Carlo.

The bottom plot of Figure 6.1 gives the ratio of efficiencies for data and Monte Carlo. The ratios are summarized in Table 6.1. Since there is a disparity between electron identification efficiency in data and Monte Carlo, the likelihood function (Eq. 4.5) used in the fit is modified. Each Monte Carlo event is weighted according to the values of the ratios of the efficiencies. The likelihood function is expressed as

$$
\mathcal{L}=\prod_{i=1}^{n}\left[\left(n-n_{B}\right) \frac{\sum_{\tilde{y}_{j} i n V_{i}} W\left(y_{j} ; \mu\right) w\left(p_{e}\right)}{\sum_{j=1}^{m} W\left(y_{j} ; \mu\right) w\left(p_{e}\right) V_{i}}+\frac{n_{i}}{V_{i}^{B}}\right]
$$

where $w\left(p_{e}\right)=\varepsilon_{\text {data }} / \varepsilon_{M C}$ is the weight due to the electron identification efficiency given in Table 6.1 and $p_{e}$ is the electron momentum.

\subsection{Hadron Identification}

A similar investigation of the identification efficiency in the Monte Carlo and data was done for the remaining two charged tracks in the decay: $K^{-}$and $\pi^{+}$. In this analysis hadrons are identified in the Čerenkov counters using the probability algorithm 

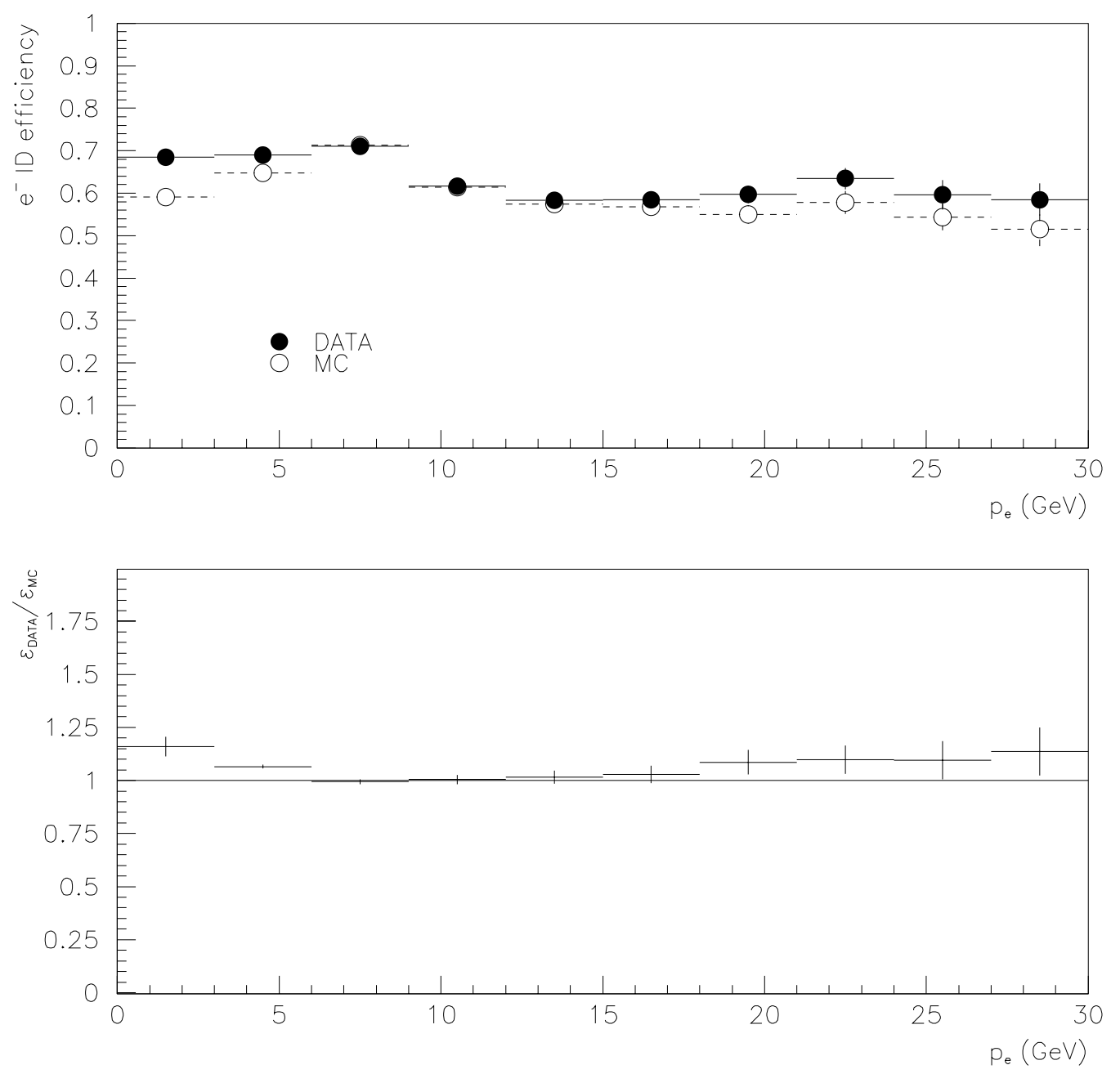

Figure 6.1: Comparison of the electron identification efficiency for EMPROB $>80$ as a function of the electron momentum for data and Monte Carlo. 
discussed in Sec. 2.4.

Data and Monte Carlo samples of $D^{+} \rightarrow K^{-} \pi^{+} \pi^{+}$decays were used to test the Čerenkov identification efficiency. This particular decay mode is optimal since it allows particle identification solely on the basis of the track charge. A sample of kaons is chosen from $D^{+}$candidates lying in the signal region $\left(1.845<M_{K \pi \pi}<1.895\right.$ $\mathrm{GeV})$. The background effects are subtracted out using the sideband region $(1.785<$ $M_{K \pi \pi}<1.81 \mathrm{GeV}$ and $\left.1.93<M_{K \pi \pi}<1.955 \mathrm{GeV}\right)$. Figure 6.2 shows the efficiency as a function of kaon momentum for kaon probability $>0.4$ (the a priori probability for a kaon is assigned to be 0.12), for both the Monte Carlo and data. The ratio of the efficiencies is plotted in the bottom figure, and summarized in Table 6.2. There is quite a discrepancy between data and Monte Carlo efficiencies for high momentum kaons.

Likewise $\pi$ Čerenkov identification is studied. The same signal and background regions as in the kaon study are used. An additional cut on candidate $D^{+}$lifetime $\left(\tau_{D}>0.934 \times 10^{-12} s\right)$ is made in order to remove feedthrough from possible $D_{s}^{+} \rightarrow$ $K^{+} K^{-} \pi^{+}$background. Both pion tracks from the $D^{+}$decay are used in the study. The top plot in Figure 6.3 shows the $\pi$ identification efficiency as a function of the $\pi$ momentum for a $\pi$ probability $>0.4$. (The a priori probability for a pion is assigned to be 0.81 .) The ratio of the efficiencies for data and Monte Carlo are plotted in the bottom plot and are summarized in Table 6.2.

As before, the likelihood function used in the fit must be amended to correct for the inaccuracies in the Monte Carlo. Including weights to accommodate the difference in data and Monte Carlo $K$ and $\pi$ identification efficiencies, the likelihood function is

$$
\mathcal{L}=\prod_{i=1}^{n}\left[\left(n-n_{B}\right) \frac{\sum_{\tilde{y}_{j} i n V_{i}} W\left(y_{j} ; \mu\right) w_{e}\left(p_{e}\right) w_{K}\left(p_{K}\right) w_{\pi}\left(p_{\pi}\right)}{\sum_{j=1}^{m} W\left(y_{j} ; \mu\right) w_{e}\left(p_{e}\right) w_{K}\left(p_{K}\right) w_{\pi}\left(p_{\pi}\right) V_{i}}+\frac{n_{i}}{V_{i}^{B}}\right]
$$

where $w_{\alpha}=\varepsilon_{\text {data }}^{\alpha} / \varepsilon_{M C}^{\alpha}$ represents the weight due to the identification efficiency of the particle of type $\alpha$ for the particle momentum $p_{\alpha}$. 

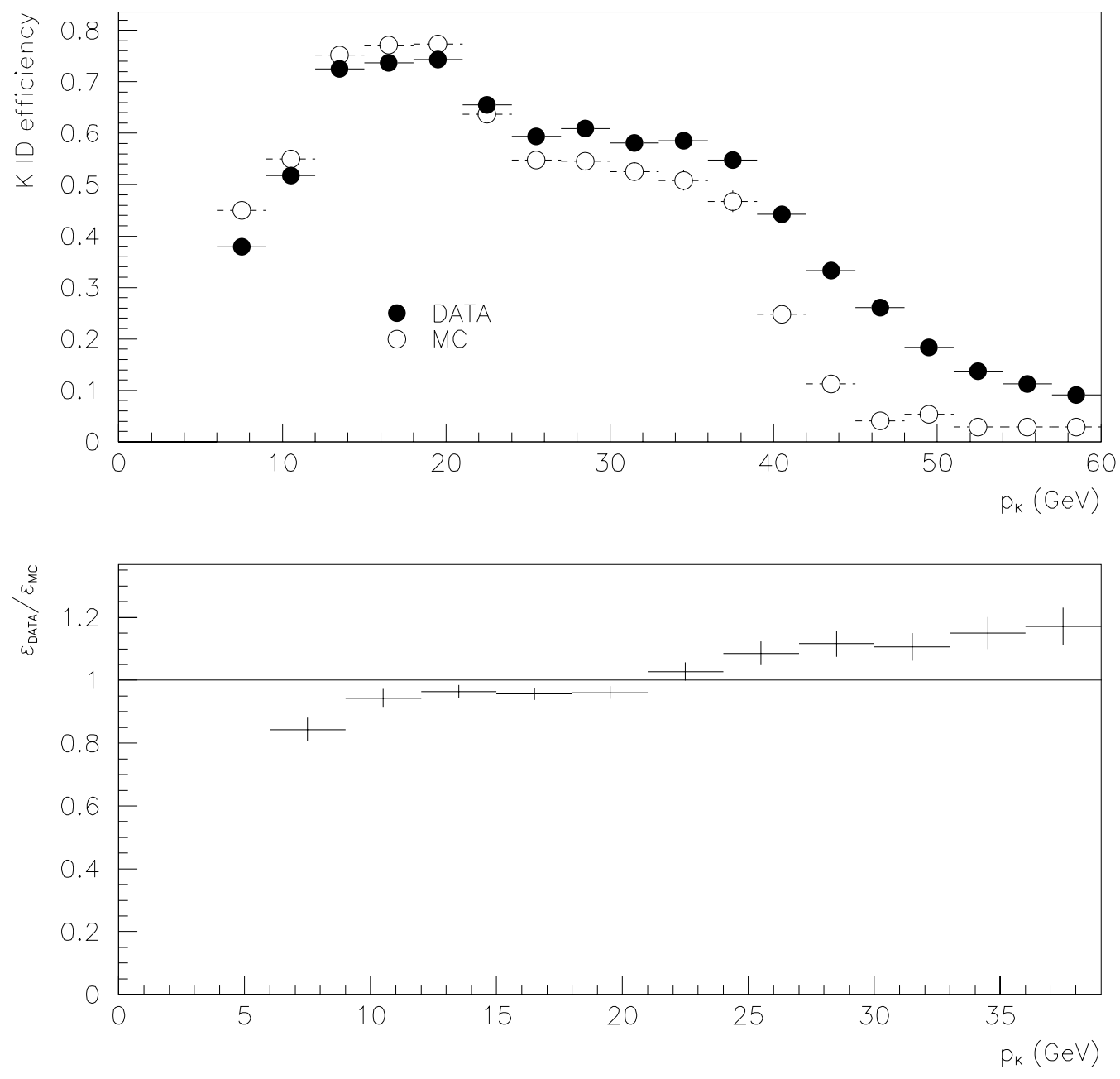

Figure 6.2: Comparison of the kaon identification efficiency (K Prob > 0.4) as a function of the kaon momentum for data and Monte Carlo. Note that only kaons with momentum less than $40 \mathrm{GeV}$ are plotted in the bottom plot 


\begin{tabular}{|c|c|c|}
\hline Momentum Range $(\mathrm{GeV})$ & $K$ Weight & $\pi$ Weight \\
\hline $0-3$ & - & $0.999 \pm 0.022$ \\
$3-6$ & - & $1.003 \pm 0.009$ \\
$6-9$ & $0.843 \pm 0.038$ & $1.086 \pm 0.010$ \\
$9-12$ & $0.943 \pm 0.030$ & $1.153 \pm 0.012$ \\
$12-15$ & $0.965 \pm 0.020$ & $1.288 \pm 0.019$ \\
$15-18$ & $0.956 \pm 0.019$ & $1.303 \pm 0.020$ \\
$18-21$ & $0.961 \pm 0.020$ & $1.303 \pm 0.021$ \\
$21-24$ & $1.027 \pm 0.029$ & $1.236 \pm 0.020$ \\
$24-27$ & $1.086 \pm 0.038$ & $1.255 \pm 0.023$ \\
$27-30$ & $1.116 \pm 0.041$ & $1.252 \pm 0.025$ \\
$30-33$ & $1.106 \pm 0.044$ & $1.269 \pm 0.028$ \\
$33-36$ & $1.150 \pm 0.051$ & $1.332 \pm 0.034$ \\
$36-39$ & $1.172 \pm 0.059$ & $1.274 \pm 0.034$ \\
$39-42$ & $1.787 \pm 0.148$ & $1.315 \pm 0.039$ \\
$42-45$ & $2.961 \pm 0.389$ & $1.240 \pm 0.035$ \\
$45-48$ & $6.489 \pm 1.580$ & $1.214 \pm 0.039$ \\
$48-51$ & $3.403 \pm 0.765$ & $1.240 \pm 0.044$ \\
$51-54$ & $4.690 \pm 1.524$ & $1.210 \pm 0.040$ \\
$54-57$ & $3.912 \pm 1.363$ & $1.227 \pm 0.048$ \\
$>57$ & $3.173 \pm 1.180$ & $1.202 \pm 0.049$ \\
\hline
\end{tabular}

Table 6.2: Weights correcting the inaccuracies in the Monte Carlo $K$ and $\pi$ identification efficiency for different ranges of track momentum. For both particles the probability cut is 0.4 

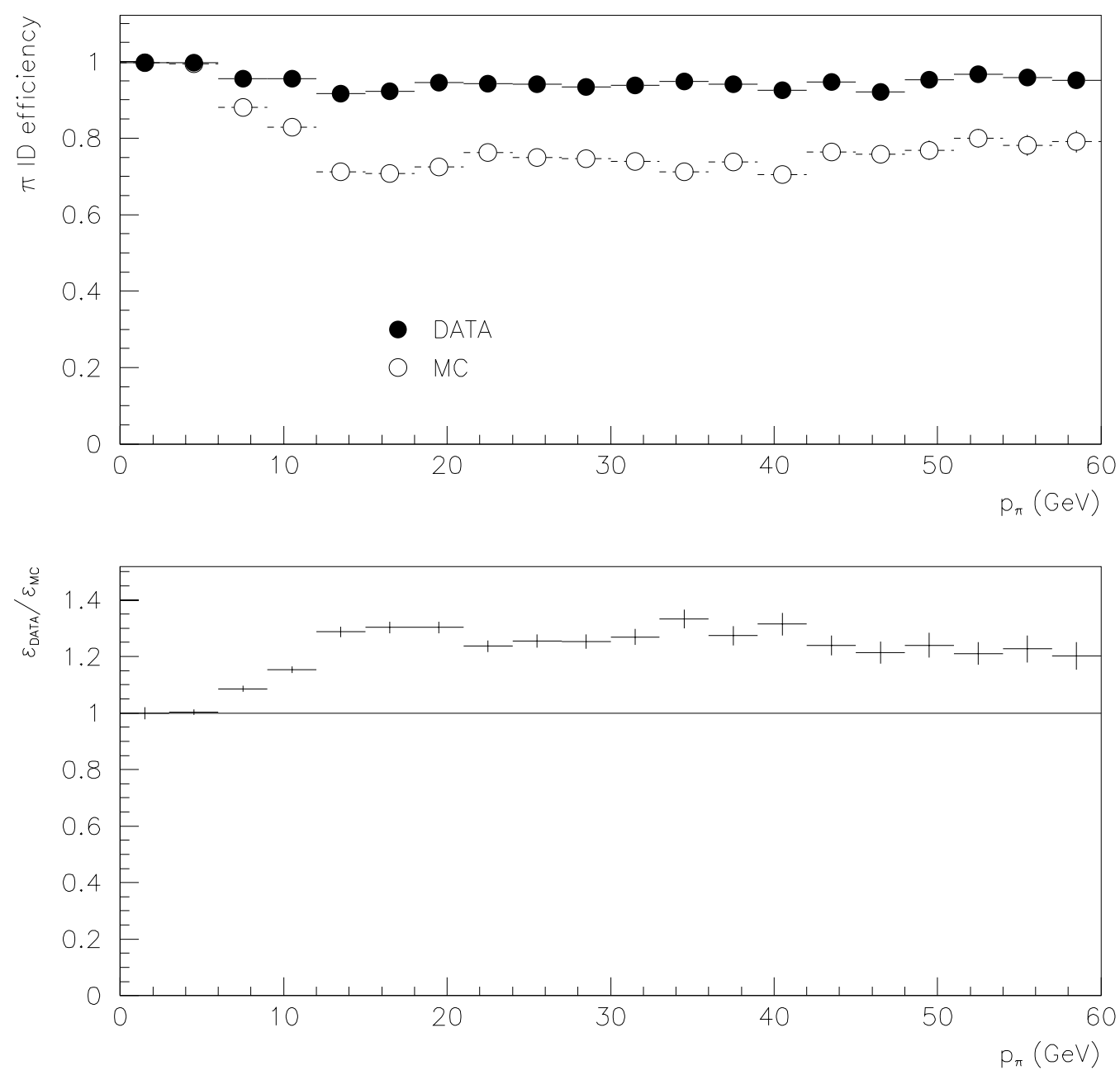

Figure 6.3: Comparison of the pion identification efficiency $(\pi$ Prob $>0.4)$ as a function of the pion momentum for data and Monte Carlo. 


\section{Chapter 7}

\section{Systematic Checks}

In this analysis we make a number of assumptions, specifically that the detector is accurately simulated (or at least the inaccuracies can be corrected for) in the Monte Carlo; that the wrong-sign events are a good representation of the background; and that the fitting technique accurately estimates the form factor ratios. A number of systematic checks to test these assumptions were performed and will be discussed in this chapter. From these checks we estimate the contribution to the total systematic uncertainty due to each source. The most significant of these are summarized in Sec. 7.4, Table 7.8. The systematic checks fall into two categories: tests of the data selection, which examine the accuracy of the Monte Carlo simulation and the wrongsign assumption; and tests of the fitting procedure.

\subsection{Fit with Known Form Factors}

A test of the fitting procedure was performed using a sample of Monte Carlo events as signal. Since the values of the form factors used in the generation of Monte Carlo events is known, we can test the performance of the fitting procedure looking, in particular, for bias in the results. The generated values of the form factor ratios are $r_{2}=0.82$ and $r_{V}=2.00$. The large Monte Carlo sample was divided into 20 subsamples of 3600 events each (approximately the same as the number of right-sign data events). Each was fit with the remaining MC events, assuming no background. 
Assuming that there is no bias in the fitting technique, the fit should return, on average, the generated values.

The distributions of fit values of $r_{2}$ and $r_{V}$ from the 20 fits are plotted in Figure 7.1. The mean values are $\left\langle r_{2}\right\rangle=0.75$ with an RMS of 0.07 , and $\left\langle r_{V}\right\rangle=2.15$ with an RMS of 0.10. The error on the means is $\sim \sigma / \sqrt{N}$ or 0.015 for $\left\langle r_{2}\right\rangle$, and 0.023 for $\left\langle r_{V}\right\rangle$. Hence, the shifts are $\delta r_{2}=-0.07 \pm 0.02$ and $\delta r_{V}=+0.15 \pm 0.02$. Thus, the means are significantly shifted away from the generated values. The shifts are comparable to the statistical errors on the values from the data. The RMS values compare well with the errors from the fit for the form factors, $\sigma_{r_{2}}=0.08$, and $\sigma_{r_{V}}=0.11$, considering that $\sim 3000$ net signal events were fit, and data includes $\sim 17 \%$ background. Also plotted in Figure 7.1 are the distributions of $\left(r^{f i t}-r^{g e n}\right) / \sigma_{r}$ for the two form factors, fit to a Gaussian. Each of these distributions should have a mean value of 0 with RMS of 1 . The actual distribution for $r_{2}$ has a mean value of $-0.93 \pm 0.29$, and a width of $1.09 \pm 0.33$. The distribution for $r_{V}$ shows a somewhat larger positive shift of $1.62 \pm 0.25$, and a width of $0.95 \pm 0.29$. Therefore, there is a systematic downward shift in the values for $r_{2}$, and an upward shift for $r_{V}$, that needs to be accounted for in the estimation of the form-factor ratios.

The values for the biases $\delta r_{2}$ and $\delta r_{V}$ are not affected by the choice of data selection criteria. The value for $\delta r_{2}$ is different when extracted with the positive neutrino momentum solution as discussed in Sec. 7.2.5. Also, it was found that the values for $\delta r_{2}$ and $\delta r_{V}$ are strongly dependent on the size of volume used for associating Monte Carlo events with each data point in the fitting technique. This is addressed in Sec. 7.3.2. To correct for the systematic bias of the fitting technique, $\delta r_{2}$ and $\delta r_{V}$ are added to the the values for the form-factor ratios returned by the fit. We assign no systematic error due to this correction. 

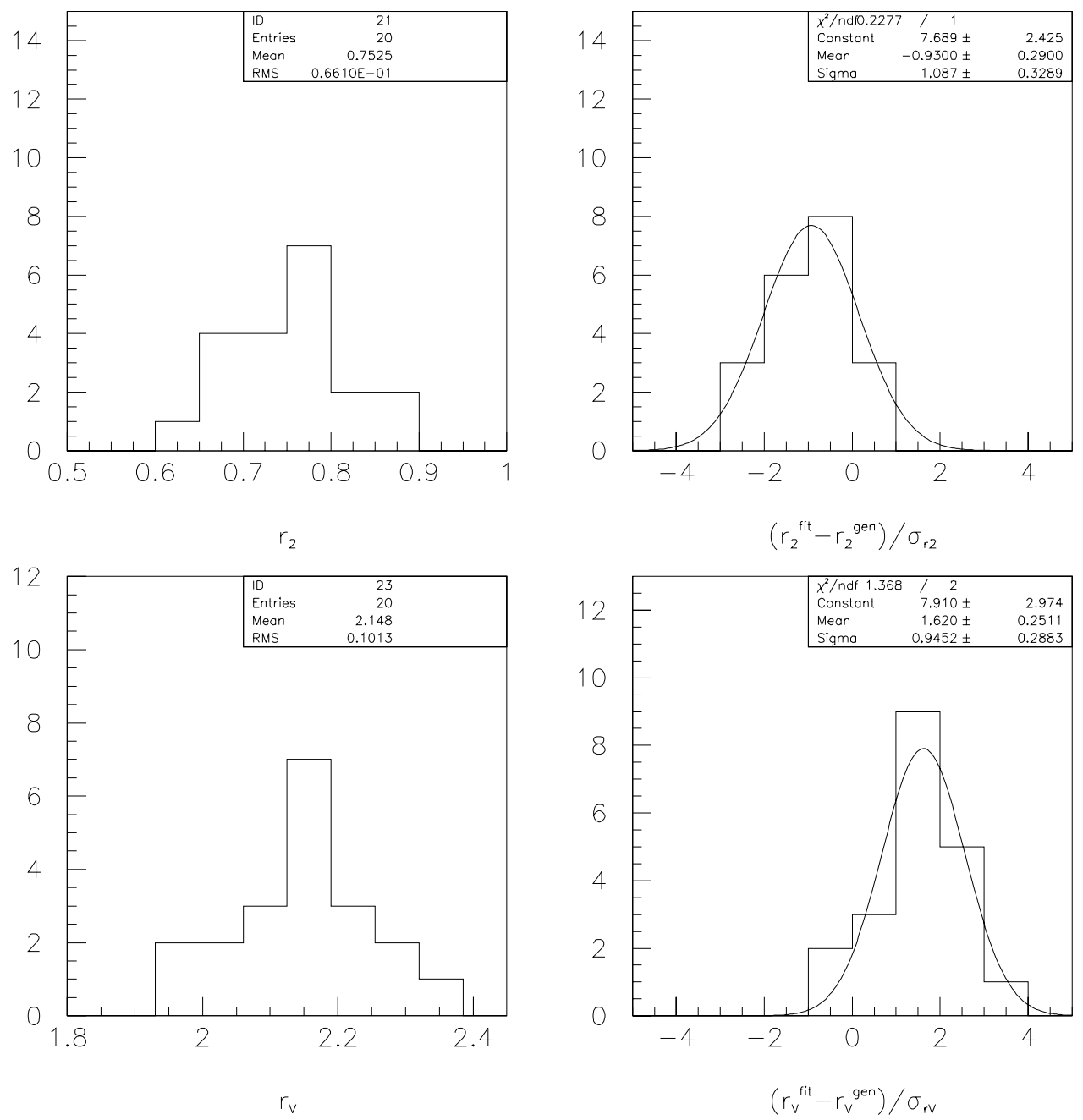

Figure 7.1: Distributions of form factor ratios determined from 20 fits of Monte Carlo samples approximately the same size as the right-sign data sample. 


\subsection{Checks of Data Selection}

\subsubsection{Final Cut Selection}

As mentioned previously, in the discussion of the selection of the final set of cuts, CART was tuned on fifteen small samples of data. The form-factor ratios extracted from fits to the 15 data samples selected by the different sets of cuts are summarized in Table 7.1 and plotted in Figure 7.2. The average values for the form factor ratios from the fifteen fits are $\left\langle r_{2}\right\rangle=0.67$ and $\left\langle r_{V}\right\rangle=1.99$. The set of cuts among those with results closest to the mean values that also produces the highest $S / \sqrt{S+B}$, where $B$ is the number of wrong-sign events and $S$ is the difference in the numbers of right-sign and wrong-sign events, for the entire data set was chosen for the nominal result. That set of cuts is listed as "Cut 4" in Table 7.1. It is expected that all fifteen samples should produce results that are consistent with each other, since they have many events in common. That appears to be the case. Also listed in the table is a "Straight" cut, which represents the set of cuts used in a previous iteration of this analysis [1]. ${ }^{1}$ This set of cuts does not use linear combinations of cut parameters, unlike the 15 sets of cuts generated with CART.

The systematic uncertainty due to the choice of selection criteria is extracted from the RMS of the distribution for the values of $r_{2}$ and $r_{V}$ with the 15 sets of CART cuts after subtracting the contribution expected from statistical differences in the sample. To estimate this contribution we use the results of a similar Monte Carlo study [38] which uses a sample of Monte Carlo events as signal. The RMS for $r_{2}$ is 0.04; for $r_{V}$, it is 0.06 . The RMS from the Monte Carlo study for $r_{2}$ is 0.03 ; for $r_{V}$ it is 0.05 . Subtracting these values in quadrature from those listed above for data, we arrive at the estimate for the systematic uncertainty due to our choice of selection criteria, free of purely statistical effects.

\footnotetext{
${ }^{1}$ The cut on SDIP was modified.
} 


\begin{tabular}{|c|c|c|c|c|c|}
\hline Cut & Right-Sign & Wrong-Sign & Monte Carlo & $r_{2}$ & $r_{V}$ \\
\hline \multicolumn{6}{|c|}{ CART Cuts } \\
\hline 1 & 3461 & 547 & 72005 & $0.68 \pm 0.08$ & $1.94 \pm 0.11$ \\
\hline 2 & 3586 & 614 & 72723 & $0.71 \pm 0.08$ & $2.02 \pm 0.11$ \\
\hline 3 & 3373 & 541 & 66743 & $0.72 \pm 0.08$ & $2.08 \pm 0.12$ \\
\hline$\star 4 \star$ & 3595 & 602 & 73161 & $0.64 \pm 0.08$ & $1.99 \pm 0.11$ \\
\hline 5 & 3651 & 654 & 70172 & $0.74 \pm 0.08$ & $2.08 \pm 0.11$ \\
\hline 6 & 3141 & 430 & 65502 & $0.66 \pm 0.08$ & $1.93 \pm 0.11$ \\
\hline 7 & 3690 & 700 & 73434 & $0.65 \pm 0.08$ & $1.97 \pm 0.11$ \\
\hline 8 & 3092 & 426 & 65900 & $0.59 \pm 0.09$ & $1.99 \pm 0.12$ \\
\hline 9 & 3231 & 451 & 67155 & $0.70 \pm 0.08$ & $1.88 \pm 0.11$ \\
\hline 10 & 3846 & 728 & 76102 & $0.62 \pm 0.08$ & $2.00 \pm 0.11$ \\
\hline 11 & 3326 & 546 & 66354 & $0.70 \pm 0.08$ & $1.98 \pm 0.11$ \\
\hline 12 & 3655 & 668 & 74636 & $0.67 \pm 0.08$ & $1.95 \pm 0.11$ \\
\hline 13 & 3355 & 530 & 68870 & $0.64 \pm 0.08$ & $2.00 \pm 0.11$ \\
\hline 14 & 3389 & 473 & 68955 & $0.68 \pm 0.08$ & $2.06 \pm 0.11$ \\
\hline 15 & 3578 & 666 & 73963 & $0.59 \pm 0.08$ & $1.96 \pm 0.11$ \\
\hline $\begin{array}{c}\text { Average } \\
\text { RMS }\end{array}$ & & & & $\begin{array}{c}0.67 \pm 0.01 \\
0.04\end{array}$ & $\begin{array}{c}1.99 \pm 0.01 \\
0.06\end{array}$ \\
\hline \multicolumn{6}{|c|}{ "Straight" Cut } \\
\hline & 3006 & 611 & 65814 & $0.53 \pm 0.10$ & $2.04 \pm 0.13$ \\
\hline
\end{tabular}

Table 7.1: Results for the form factor ratios from 15 different sets of CART cuts. Cut 4 is chosen as "standard". "Straight" cut refers to the set of cuts used in a previous version of this analysis. (Refer to the text for more details). The values listed here have not yet been corrected for a systematic bias due to the fitting technique. 


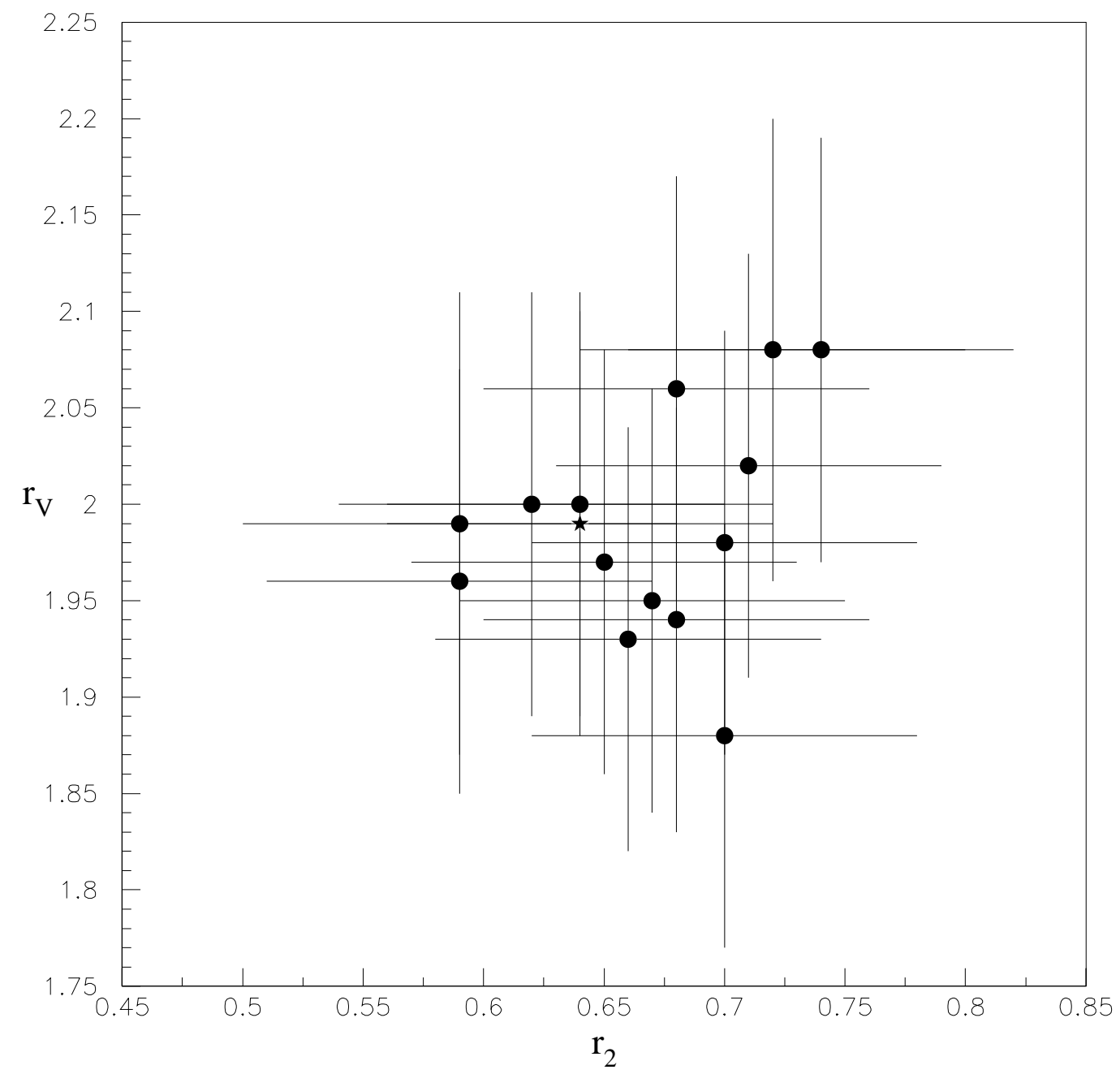

Figure 7.2: Results for the form factor ratios with 15 different sets of CART cuts. The form-factor ratios used for the final result are designated with a $\operatorname{star}(\star)$. The values have not yet been corrected for a systematic bias due to the fitting technique. 


\begin{tabular}{|l|c|c|c|c|c|}
\hline & Right-Sign & Wrong-Sign & Monte Carlo & $r_{2}$ & $r_{V}$ \\
\hline \multicolumn{6}{|c|}{ Run Number } \\
\hline 1st 1/4 & 525 & 93 & 73161 & $0.56 \pm 0.22$ & $2.04 \pm 0.30$ \\
2nd 1/4 & 1045 & 175 & 73161 & $0.49 \pm 0.16$ & $2.20 \pm 0.23$ \\
3rd 1/4 & 1060 & 165 & 73161 & $0.88 \pm 0.14$ & $1.87 \pm 0.19$ \\
4th 1/4 & 965 & 169 & 73161 & $0.67 \pm 0.15$ & $2.04 \pm 0.21$ \\
\hline \multicolumn{6}{|c|}{ Four-Target Data } \\
\hline All data & 3595 & 602 & 73161 & $0.64 \pm 0.08$ & $1.99 \pm 0.11$ \\
5-target only & 3471 & 577 & 73161 & $0.67 \pm 0.08$ & $2.00 \pm 0.11$ \\
\hline \multicolumn{6}{|c|}{ D Charge } \\
\hline$D^{+}$only & 1576 & 285 & 19849 & $0.65 \pm 0.12$ & $2.01 \pm 0.17$ \\
$D^{-}$only & 2019 & 317 & 53312 & $0.71 \pm 0.11$ & $2.05 \pm 0.15$ \\
\hline
\end{tabular}

Table 7.2: Results of systematic checks of data selection. The values listed here have not yet been corrected for a systematic bias due to the fitting technique.

\subsubsection{Fitting Subsamples of the Data}

In order to study time-dependent effects on the results for the form factor-ratios, the data was divided into four subsets based on run number, and each subset was fit with the large Monte Carlo sample. The first subset consists of runs 678 - 909; the second of runs 910 - 1141; the third of runs 1142 - 1374; and the fourth of runs 1375 - 1606. The four subsamples are completely independent. The fit results, given in Table 7.2, are consistent with one another, and with the results of the fit to the entire data sample, as expected. No systematic error is assigned for the observed spread in form-factor ratios.

\subsubsection{Four-Target Data}

Early in E791 data-taking, only four targets were used instead of five. A check was made of the effect events prior to run 780 have on the values for the form factor ratios. A fit performed after these events were removed from the data sample shows little effect on the values of the form factor ratios. The results of this check are listed in Table 7.2. No systematic error is assigned for the observed change in form-factor 


\begin{tabular}{|l|c|c|c|c|}
\hline$p_{\nu}$ Solution & $\delta r_{2}$ & $r_{2}$ & $\delta r_{V}$ & $r_{V}$ \\
\hline Negative & -0.07 & $0.71 \pm 0.08$ & +0.15 & $1.84 \pm 0.11$ \\
Positive & -0.13 & $0.61 \pm 0.09$ & +0.14 & $1.84 \pm 0.12$ \\
\hline
\end{tabular}

Table 7.3: Results for the fits with both choices for the neutrino momentum. The appropriate bias due to the fitting technique is incorporated into the values for the form-factor ratios.

ratios.

\subsubsection{Charge Dependence}

Fits were performed separately for the $D^{+}$and $D^{-}$samples. There are $\sim 3$ times as many $D^{-}$as $D^{+}$events in the final Monte Carlo sample. Each subset of the data was fit with the subsample of the Monte Carlo with the corresponding $D$ charge. The results of the fits are listed in Table 7.2. The difference between the fit values for $D^{+}$candidates and $D^{-}$candidates for $r_{2}$ is $0.06 \pm 0.16$, and for $r_{V}$ is $0.04 \pm 0.23$. No systematic error is assigned for this difference.

\subsubsection{Choice of Neutrino Momentum}

Since the neutrino in the $D^{+} \rightarrow \bar{K}^{* 0} e^{+} \nu_{e}$ decay cannot be detected, we calculate the neutrino momentum from the measurements of the momenta of the visible tracks, with the assumption that the decaying particle is a $D^{+}$. Still we are left with quadratic ambiguity in the choice of the longitudinal component of the neutrino momentum (see Sec. 4.4). In this analysis we always use the negative solution for $p_{\text {long }}^{\nu}$, as did E653 [16] and E691 [15]. The misreconstruction of the neutrino momentum leads to the kinematic variables also being reconstructed incorrectly. Separate fits were performed using the negative solution and the positive solution for both Monte Carlo and data. The results of the fits are summarized in Table 7.3. The bias in $r_{2}$ is significantly different for the two solutions. The results for the form factor ratios with the two different solutions are consistent with each other considering that the data samples with the two solutions are partially statistically independent. Each 
time the correct solution is used for an event, (and hence the correct position in the kinematic variable space is assigned) in one sample, the wrong solution is used in the other sample. Only in such cases where there is an unphysical result for $p_{\text {long }}^{\nu}$, the data event enters both samples at the same value of $p_{\nu}$ and is identical in both samples. No systematic uncertainty is assigned for the difference in values for the form-factor ratios.

\subsection{Checks of Fitting Technique}

\subsubsection{Number of Monte Carlo Points Associated with Data}

In the fit, the number of Monte Carlo points lying in a small five-dimensional volume of kinematic variable space surrounding each data point is used to determine the likelihood. Data events with less than four Monte Carlo events lying in the volume around it are excluded from the fit. In the limit of infinite Monte Carlo statistics, the fit estimates for the form factor ratios should be stable regardless of the minimum number of points required. Since we have a finite number of Monte Carlo events two possible concerns arise. If too few Monte Carlo events are required, this would fail to account for the statistical fluctuations in the Monte Carlo. On the other hand, requiring too many Monte Carlo points, would cause a large number of data events

to be excluded from the fit. A check to test the dependence of the fit results on the minimum number of Monte Carlo points required to lie in the volume $V_{i}$ was done. The results of this study are summarized in Table 7.4. We chose to require four Monte Carlo points, since it avoids both of the two extremes. The assigned systematic errors for this source are $\sigma_{r_{2}}=0.01$ and $\sigma_{r_{V}}=0.01$.

\subsubsection{Monte Carlo Volume Size}

The volume used in the fit must not be too large, such that there would be too wide a region of kinematic variable space associated with each data point, and thus the fit would lose any sensitivity to the form factors. On the other hand, a volume that is too small would also fail to represent the true distributions of the kinematic variables 


\begin{tabular}{|c|c|c|c|}
\hline \# MC events req. & \# Data events excl. & $r_{2}$ & $r_{V}$ \\
\hline 1 & 34 & $0.64 \pm 0.08$ & $2.00 \pm 0.11$ \\
2 & 68 & $0.64 \pm 0.08$ & $1.99 \pm 0.11$ \\
3 & 97 & $0.64 \pm 0.08$ & $1.99 \pm 0.11$ \\
4 & 129 & $0.64 \pm 0.08$ & $1.99 \pm 0.11$ \\
5 & 154 & $0.63 \pm 0.08$ & $1.99 \pm 0.11$ \\
6 & 170 & $0.63 \pm 0.08$ & $1.99 \pm 0.11$ \\
7 & 195 & $0.63 \pm 0.08$ & $1.99 \pm 0.11$ \\
\hline
\end{tabular}

Table 7.4: Results of the study of the dependence of the fit on the minimum number of Monte Carlo points required to lie in a small volume surrounding each data point. The second column lists the number of data points failing this requirement, and thus excluded from the fit.

due to large statistical fluctuations in the number of Monte Carlo events. But it is expected that for a range of volume sizes, the fit results should be stable.

A check was performed to study the sensitivity of the fit to the volume size. The full range of each kinematic variable is $\cos \theta_{l}=[-1,1], \cos \theta_{V}=[-1,1], q^{2} / q_{\max }^{2}=$ $[0,1]$, and $\chi=[0,2 \pi]$. A nominal size of $1 / 625$ of the total volume $\left(\Delta \cos \theta_{l}=\right.$ $\left.0.40, \Delta \cos \theta_{V}=0.40, \Delta q^{2} / q_{\max }^{2}=0.20, \Delta \chi=1.26\right)$ was chosen. Then the range was varied from 1 to $1 / 9$ of the total range for one variable at a time, holding the other three ranges constant. The results of the fits are plotted in Figure 7.3, and summarized in Tables 7.5 and 7.6, along with the values of the biases for $r_{2}$ and $r_{V}$ due to the fitting technique determined from the Monte Carlo for each case. The fifth point from the left (open circle) represents the nominal volume size used for the quoted fit results. It was found that biases $\delta r_{2}$ and $\delta r_{V}$ due to the fitting technique are dependent on the choice of the volume size, especially for $\cos \theta_{V}$. Once the biases are corrected for, the fit is approximately stable for the following ranges: $0.22<$ $\Delta \cos \theta_{l}<0.67 ; 0.22<\Delta \cos \theta_{V}<0.50 ; 0.11<\Delta q^{2} / q_{\max }^{2}<0.25 ; 0.70<\Delta \chi<2.09$. The systematic error dues to this source is determined from the spread in values for the form-factor ratios in the range $1 / 1125$ to $1 / 375$ of the total five-dimensional volume. The assigned uncertainties are $\sigma_{r_{2}}=0.04$ and $\sigma_{r_{V}}=0.04$. 

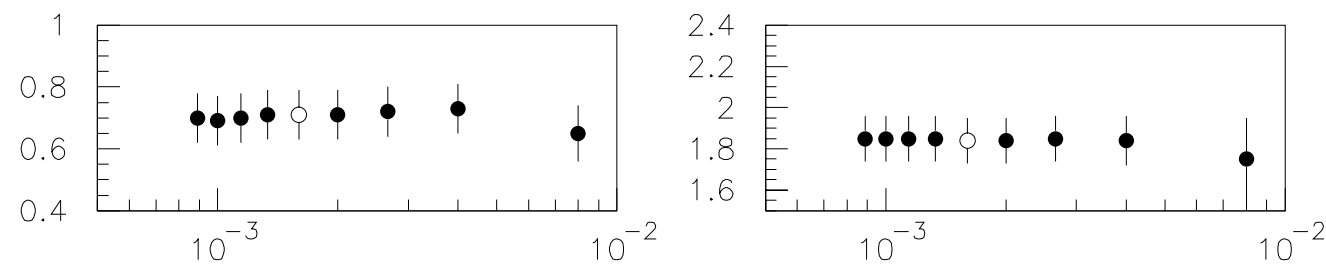

$r_{2}$ v. MC Volume Size

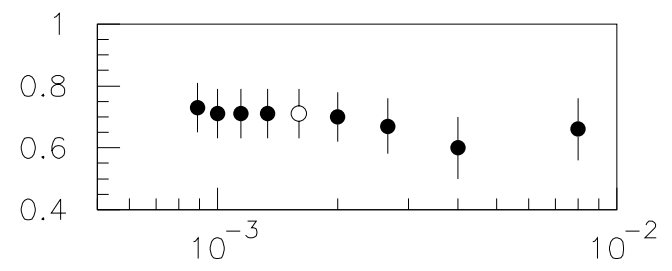

$r_{v} v$. MC Volume Size

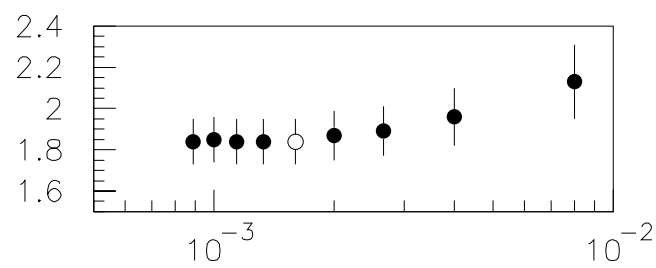

$r_{2}$ v. MC Volume Size

rv v. MC Volume Size
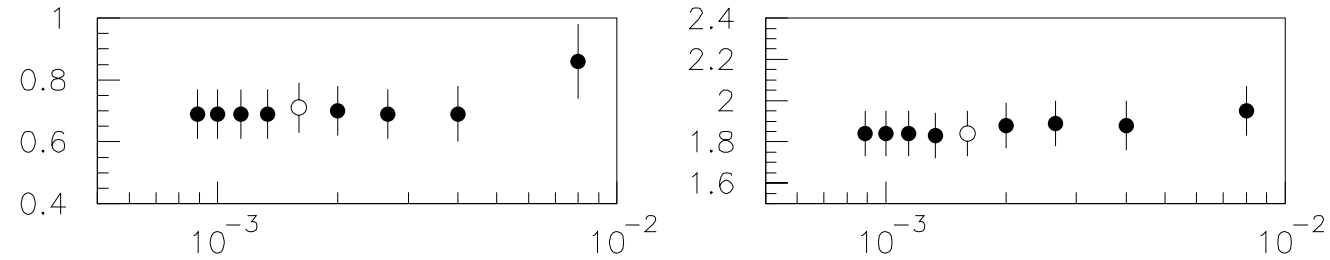

$r_{2}$ v. MC Volume Size

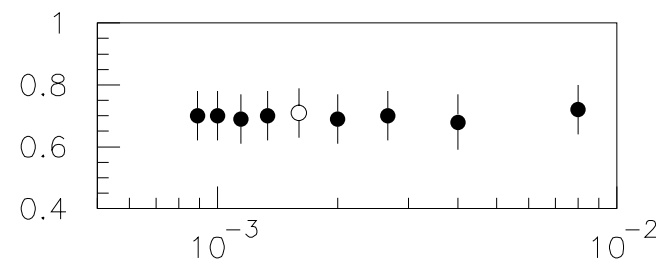

rvv. MC Volume Size

$r_{2}$ v. MC Volume Size

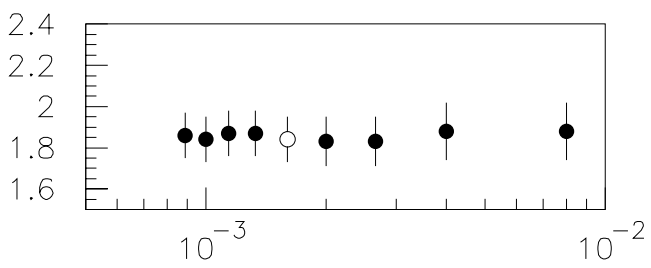

$r_{v} v . M C$ Volume Size

Figure 7.3: Fit estimates for the form factor ratios as the range in one kinematic variable is varied, with the other four being held constant. In the top two plots the range in $\cos \theta_{l}$ is varied; next two plots show the variation in $\cos \theta_{V}$; then $q^{2} / q_{\text {max }}^{2}$; and $\chi$ in the bottom plots. The volume size is expressed as the fraction of the total fivedimensional volume. The systematic bias due the the fitting technique is corrected for. 


\begin{tabular}{|c|c|c|c|c|c|c|c|}
\hline$\Delta \cos \theta_{l}$ & $\Delta \cos \theta_{V}$ & $\Delta q^{2} / q_{\max }^{2}$ & $\Delta \chi$ & $\delta r_{2}$ & $r_{2}$ & $\delta r_{V}$ & $r_{V}$ \\
\hline \multicolumn{7}{|c|}{ Full Range } \\
\hline 2.00 & 2.00 & 1.00 & 6.28 & - & - & - & - \\
\hline \multicolumn{7}{|c|}{ Nominal Volume } \\
\hline 0.40 & 0.40 & 0.20 & 1.26 & -0.07 & $0.71 \pm 0.08$ & +0.15 & $1.84 \pm 0.11$ \\
\hline \multicolumn{8}{|c|}{ Varying $\Delta \cos \theta_{l}$} \\
\hline 2.00 & 0.40 & 0.20 & 1.26 & -0.09 & $0.65 \pm 0.09$ & +0.24 & $1.75 \pm 0.20$ \\
1.00 & 0.40 & 0.20 & 1.26 & -0.05 & $0.73 \pm 0.08$ & +0.19 & $1.84 \pm 0.12$ \\
0.67 & 0.40 & 0.20 & 1.26 & -0.07 & $0.72 \pm 0.08$ & +0.16 & $1.85 \pm 0.11$ \\
0.50 & 0.40 & 0.20 & 1.26 & -0.07 & $0.71 \pm 0.08$ & +0.15 & $1.84 \pm 0.11$ \\
0.33 & 0.40 & 0.20 & 1.26 & -0.07 & $0.71 \pm 0.08$ & +0.14 & $1.85 \pm 0.11$ \\
0.29 & 0.40 & 0.20 & 1.26 & -0.06 & $0.70 \pm 0.08$ & +0.14 & $1.85 \pm 0.11$ \\
0.25 & 0.40 & 0.20 & 1.26 & -0.05 & $0.69 \pm 0.08$ & +0.14 & $1.85 \pm 0.11$ \\
0.22 & 0.40 & 0.20 & 1.26 & -0.05 & $0.70 \pm 0.08$ & +0.14 & $1.85 \pm 0.11$ \\
\hline \multicolumn{7}{|c|}{ Varying $\Delta \cos \theta_{V}$} \\
\hline 0.40 & 2.00 & 0.20 & 1.26 & 0.00 & $0.66 \pm 0.10$ & +0.03 & $2.13 \pm 0.18$ \\
0.40 & 1.00 & 0.20 & 1.26 & -0.39 & $0.60 \pm 0.10$ & +0.36 & $1.96 \pm 0.14$ \\
0.40 & 0.67 & 0.20 & 1.26 & -0.19 & $0.67 \pm 0.09$ & +0.23 & $1.89 \pm 0.12$ \\
0.40 & 0.50 & 0.20 & 1.26 & -0.11 & $0.70 \pm 0.08$ & +0.17 & $1.87 \pm 0.12$ \\
0.40 & 0.33 & 0.20 & 1.26 & -0.04 & $0.71 \pm 0.08$ & +0.13 & $1.84 \pm 0.11$ \\
0.40 & 0.29 & 0.20 & 1.26 & -0.03 & $0.71 \pm 0.08$ & +0.13 & $1.84 \pm 0.11$ \\
0.40 & 0.25 & 0.20 & 1.26 & -0.02 & $0.71 \pm 0.08$ & +0.12 & $1.85 \pm 0.11$ \\
0.40 & 0.22 & 0.20 & 1.26 & -0.02 & $0.73 \pm 0.08$ & +0.12 & $1.84 \pm 0.11$ \\
\hline
\end{tabular}

Table 7.5: Form factor ratios determined from the fit for various volume sizes for the variables $\cos \theta_{l}$ and $\cos \theta_{V}$. 


\begin{tabular}{|c|c|c|c|c|c|c|c|}
\hline$\Delta \cos \theta_{l}$ & $\Delta \cos \theta_{V}$ & $\Delta q^{2} / q_{\max }^{2}$ & $\Delta \chi$ & $\delta r_{2}$ & $r_{2}$ & $\delta r_{V}$ & $r_{V}$ \\
\hline \multicolumn{7}{|c|}{ Full Range } \\
\hline 2.00 & 2.00 & 1.00 & 6.28 & - & - & - & - \\
\hline \multicolumn{7}{|c|}{ Nominal Volume } \\
\hline 0.40 & 0.40 & 0.20 & 1.26 & -0.07 & $0.71 \pm 0.08$ & +0.15 & $1.84 \pm 0.11$ \\
\hline \multicolumn{10}{|c|}{ Varying $\Delta q^{2} / q_{\max }^{2}$} \\
\hline 0.40 & 0.40 & 1.00 & 1.26 & -0.19 & $0.86 \pm 0.12$ & +0.13 & $1.95 \pm 0.12$ \\
0.40 & 0.40 & 0.50 & 1.26 & -0.11 & $0.69 \pm 0.09$ & +0.14 & $1.88 \pm 0.12$ \\
0.40 & 0.40 & 0.33 & 1.26 & -0.08 & $0.69 \pm 0.08$ & +0.13 & $1.89 \pm 0.11$ \\
0.40 & 0.40 & 0.25 & 1.26 & -0.07 & $0.70 \pm 0.08$ & +0.14 & $1.88 \pm 0.11$ \\
0.40 & 0.40 & 0.17 & 1.26 & -0.06 & $0.69 \pm 0.08$ & +0.15 & $1.83 \pm 0.11$ \\
0.40 & 0.40 & 0.14 & 1.26 & -0.06 & $0.69 \pm 0.08$ & +0.15 & $1.84 \pm 0.11$ \\
0.40 & 0.40 & 0.13 & 1.26 & -0.06 & $0.69 \pm 0.08$ & +0.15 & $1.84 \pm 0.11$ \\
0.40 & 0.40 & 0.11 & 1.26 & -0.06 & $0.69 \pm 0.08$ & +0.15 & $1.84 \pm 0.11$ \\
\hline \multicolumn{8}{|c|}{ Varying $\Delta \chi$} \\
\hline 0.40 & 0.40 & 0.20 & 6.28 & -0.08 & $0.72 \pm 0.08$ & +0.11 & $1.88 \pm 0.14$ \\
0.40 & 0.40 & 0.20 & 3.14 & -0.17 & $0.68 \pm 0.09$ & +0.42 & $1.88 \pm 0.14$ \\
0.40 & 0.40 & 0.20 & 2.09 & -0.11 & $0.70 \pm 0.08$ & +0.24 & $1.83 \pm 0.12$ \\
0.40 & 0.40 & 0.20 & 1.57 & -0.08 & $0.69 \pm 0.08$ & +0.17 & $1.83 \pm 0.12$ \\
0.40 & 0.40 & 0.20 & 1.05 & -0.06 & $0.70 \pm 0.08$ & +0.13 & $1.87 \pm 0.11$ \\
0.40 & 0.40 & 0.20 & 0.90 & -0.05 & $0.69 \pm 0.08$ & +0.13 & $1.87 \pm 0.11$ \\
0.40 & 0.40 & 0.20 & 0.79 & -0.05 & $0.70 \pm 0.08$ & +0.13 & $1.84 \pm 0.11$ \\
0.40 & 0.40 & 0.20 & 0.70 & -0.04 & $0.70 \pm 0.08$ & +0.13 & $1.86 \pm 0.11$ \\
\hline
\end{tabular}

Table 7.6: Form factor ratios determined from the fit for various volume sizes for the variables $q^{2} / q_{\max }^{2}$ and $\chi$. 


\subsubsection{Background Volume Size}

A similar check was performed to study the effect of varying the size of the volume surrounding each data point that is used for associating wrong-sign "background" events. The number of wrong-sign events that lie within this volume determines the probability of the data point being background. The background volume size was varied from $1 / 16$ to $1 / 3645$ of the total volume. The total number of wrong-sign events in the data sample is 602. The fit results are plotted in Figure 7.4. There is very little effect on the value $r_{V}$ from the choice of the background volume. $r_{2}$ shows about a one-half sigma effect for volume sizes less than $10^{-3}$, which is very small, given the number of wrong-sign events. The third point from the right represents the form factor values quoted in the result. The systematic uncertainty from this source is determined from the spread in values of the form-factor ratios in the volume range $10^{-3}-5 \times 10^{-2}$. The assigned errors are $\sigma_{r_{2}}=0.05$ and $\sigma_{r_{V}}=0.04$.

\subsubsection{Number of Background Events}

A check was performed to examine the effect on the results of the fit of varying the expected number of background events in the data sample. Typically it is assumed that the number of "wrong-sign" events is a good estimate of the number of background events in the "right-sign" data. The number of "wrong-sign" events was weighted for this check, such that a weight of 0 presumes no background in the data, and a weight of 2 corresponds to an assumption of twice as many background events in the data as there are events in the "wrong-sign" sample. The weights were varied from 0 to 2. The resulting fit values for the form factor ratios are plotted in Figure 7.5. Compared to the statistical error from the fit results for $r_{V}$ do not appear to be strongly affected by the assumed number of background events in the data. On the other hand, there does seem to be a one sigma effect for $r_{2}$. The systematic uncertainty is determined from the spread in the values of the form-factor ratios in the weight range $0.5-1.25$. The assigned errors are $\sigma_{r_{2}}=0.05$ and $\sigma_{r_{V}}=0.02$. 

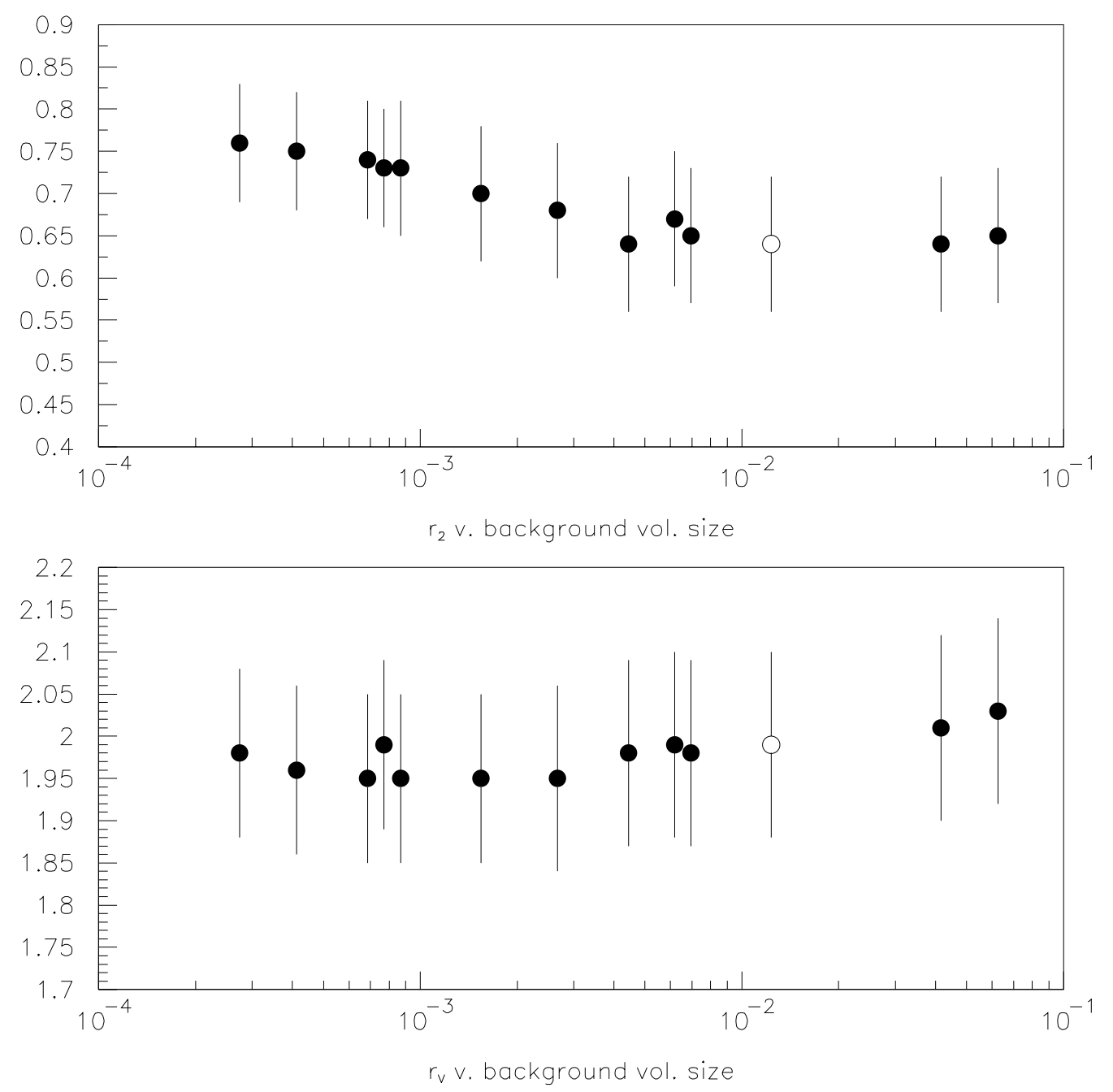

Figure 7.4: Form factor ratios determined from the fit for various background volume sizes. The volume size is expressed as a fraction of the total five-dimensional volume. 

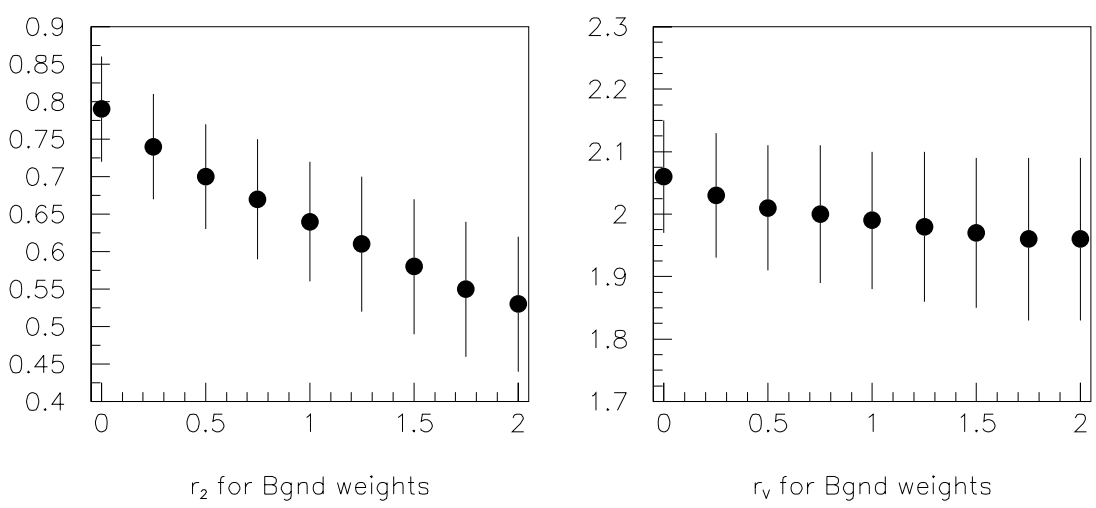

Figure 7.5: Form factor ratios determined from the fit for various background weights.

\subsubsection{Particle Identification Efficiency Correction}

A test was performed of the $e^{ \pm}, K^{ \pm}$, and $\pi^{ \pm}$identification efficiency corrections which are described in Chapter 6. We test the uncertainty on the fit estimates for the form factor ratios due to the finite size of the data and Monte Carlo samples used to derive the weights. For each of the three particle identification efficiencies 100 fits were performed where the weight associated with each momentum bin was modified by a Gaussian fluctuation with the resolution given by the error on the weight. The weights for the other two particles are kept at the nominal values. The resulting form factor ratios from these fits are plotted in Figure 7.6 for each of the three sets of 100 fits. The RMS spreads of the distributions give the uncertainties due to these effects. The mean values and the RMS of these distributions are summarized in Table 7.7. The assigned systematic error is the quadratic sum of the RMS spreads for each type of particle efficiency correction. They are $\sigma_{r_{2}}=0.01$ and $\sigma_{r_{V}}=0.05$. The systematic errors are very small compared with the statistical errors on the form factor ratios, except in the case of the error on $r_{V}$ that is due to the electron identification efficiency correction. 

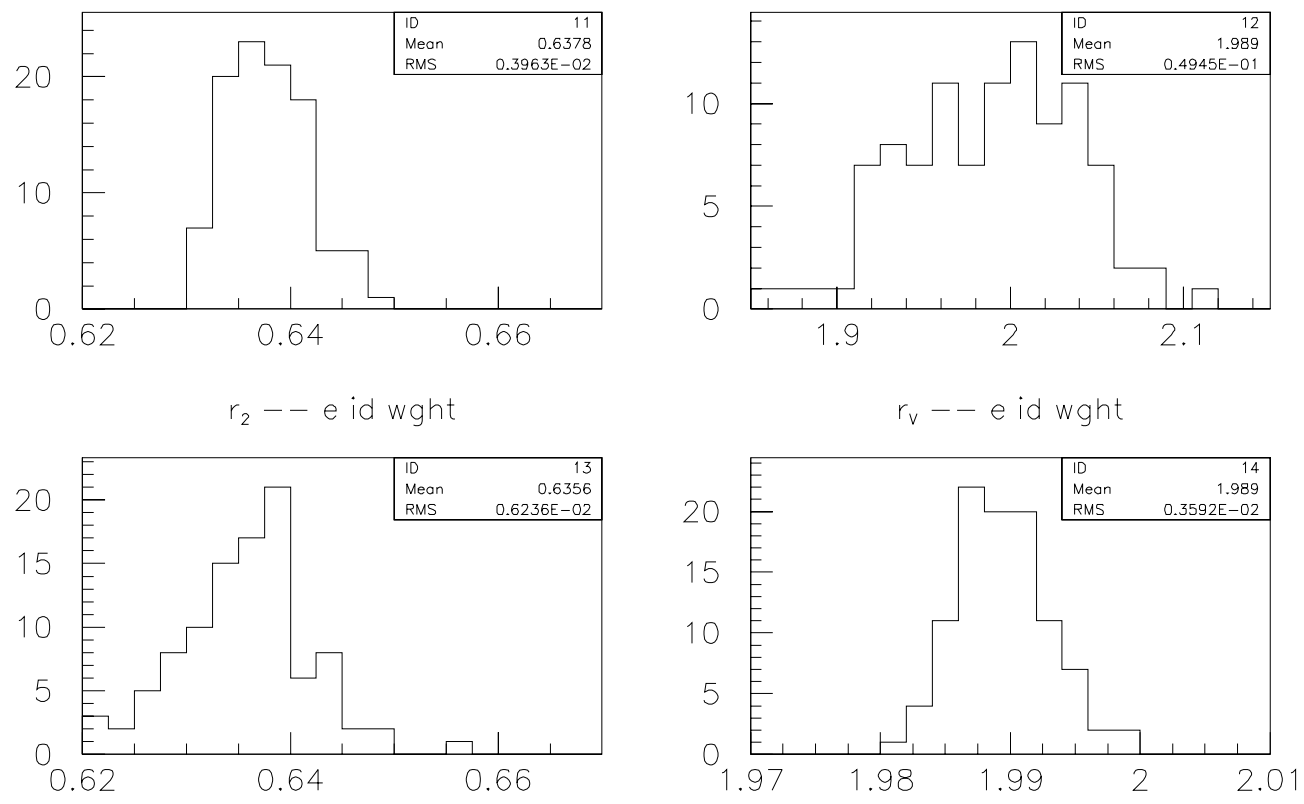

$r_{2}--$ Kid wght
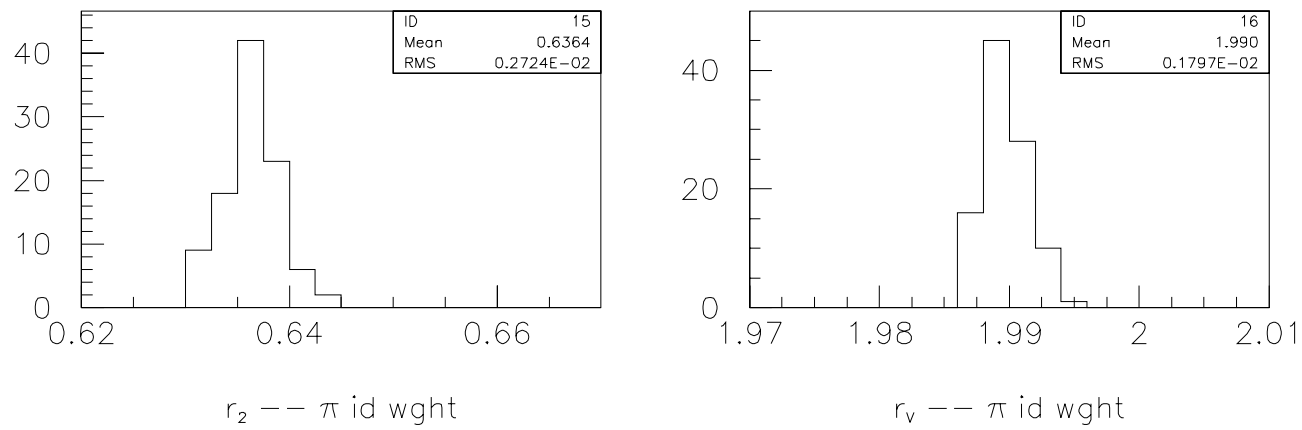

Figure 7.6: Distributions of form factor ratios determined from the 100 fits with modified electron, kaon, and pion identification efficiency weights. Note that the horizontal scale is different the top right plot. 


\begin{tabular}{|l|c|c|c|c|}
\hline Particle ID & $\left\langle r_{2}\right\rangle$ & RMS for $r_{2}$ & $\left\langle r_{V}\right\rangle$ & RMS for $r_{V}$ \\
\hline electron & 0.638 & 0.004 & 1.989 & 0.049 \\
kaon & 0.636 & 0.006 & 1.989 & 0.004 \\
pion & 0.636 & 0.003 & 1.990 & 0.002 \\
\hline
\end{tabular}

Table 7.7: Results of the checks of particle identification efficiencies.

\begin{tabular}{|l|c|c|}
\hline Source & $\sigma_{r_{2}}$ & $\sigma_{r_{V}}$ \\
\hline Cut Selection & 0.03 & 0.03 \\
Number MC pts & 0.01 & 0.01 \\
MC Volume & 0.04 & 0.04 \\
Bgnd Volume & 0.05 & 0.04 \\
Number Bgnd evts & 0.05 & 0.02 \\
Particle ID & 0.01 & 0.05 \\
\hline Total & 0.09 & 0.08 \\
\hline
\end{tabular}

Table 7.8: Contributions to the systematic uncertainty.

\subsection{Systematic Error Estimate}

The estimates of the contributions to the total systematic uncertainty on the form factor ratios due to each of the studies are listed in Table 7.8. The largest contributions to the systematic error on $r_{2}$ are related to our modeling of the background in the fit. We assume that the wrong-sign distribution wholly represents the background under the right-sign signal. The systematic error gives us some measure of our lack of understanding of the background. A major contribution to the systematic uncertainty for both $r_{2}$ and $r_{V}$ comes from the different data selection criteria, which results from the deficiencies of the detector simulation in the Monte Carlo. Individual errors are added in quadrature. The final systematic errors are comparable in size to the statistical errors on the results. 


\section{Chapter 8}

\section{Results and Discussion}

\subsection{The Form-Factor Ratios}

The results of the fit using the fitting technique described in Chapter 3 are summarized in Table 8.1 and Figure 8.1. The results for the form factor ratios are mildly anticorrelated with the correlation coefficient $\rho_{r_{2} r_{V}}=-0.127$. The systematic bias due to the fitting technique is corrected for in the results. The fit was performed on 3595 candidate $D^{+} \rightarrow \bar{K}^{* 0} e^{+} \nu_{e}$ events. The background in the data sample was modeled by 602 wrong-sign events. The volume size $V_{i}$, used to measure the density of Monte Carlo events about each data point, was chosen to be $1 / 625$ of the total fivedimensional volume. The volume size $V_{i}^{B}$, used for background density, was chosen to be $1 / 81$ of the total volume. A minimum of four Monte Carlo points were required to fall in the volume $V_{i}$.

Table 8.1 also contains the published results for $r_{2}$ and $r_{V}$ measured by other experiments. There were more events used in the E791 fit and correspondingly the statistical errors on the resulting values of $r_{2}$ and $r_{V}$ are smaller. The previously published results are quite consistent with the more precise E791 result.

We can extract the values of the form factors $A_{1}, A_{2}$, and $V$ from the ratios $r_{2}$ and $r_{V}$ using the procedure outlined in Körner et al. [40]. They give the partial decay 


\begin{tabular}{|l|c|c|c|}
\hline Group & $r_{2}$ & $r_{V}$ & Signal Events \\
\hline E791 [39] & $0.71 \pm 0.08 \pm 0.09$ & $1.84 \pm 0.11 \pm 0.08$ & 2993 \\
\hline \hline E687 [17] & $0.78 \pm 0.18 \pm 0.10$ & $1.74 \pm 0.27 \pm 0.28$ & 875 \\
E653 [16] & $0.82_{-0.23}^{+0.22} \pm 0.11$ & $2.00_{-0.32}^{+0.34} \pm 0.16$ & $\approx 275$ \\
E691 [15] & $0.0 \pm 0.5 \pm 0.2$ & $2.0 \pm 0.6 \pm 0.3$ & 183 \\
\hline Average & $0.71 \pm 0.09$ & $1.85 \pm 0.12$ & \\
\hline
\end{tabular}

Table 8.1: Experimental measurements of $r_{2}$ and $r_{V}$ by E791 and other groups.

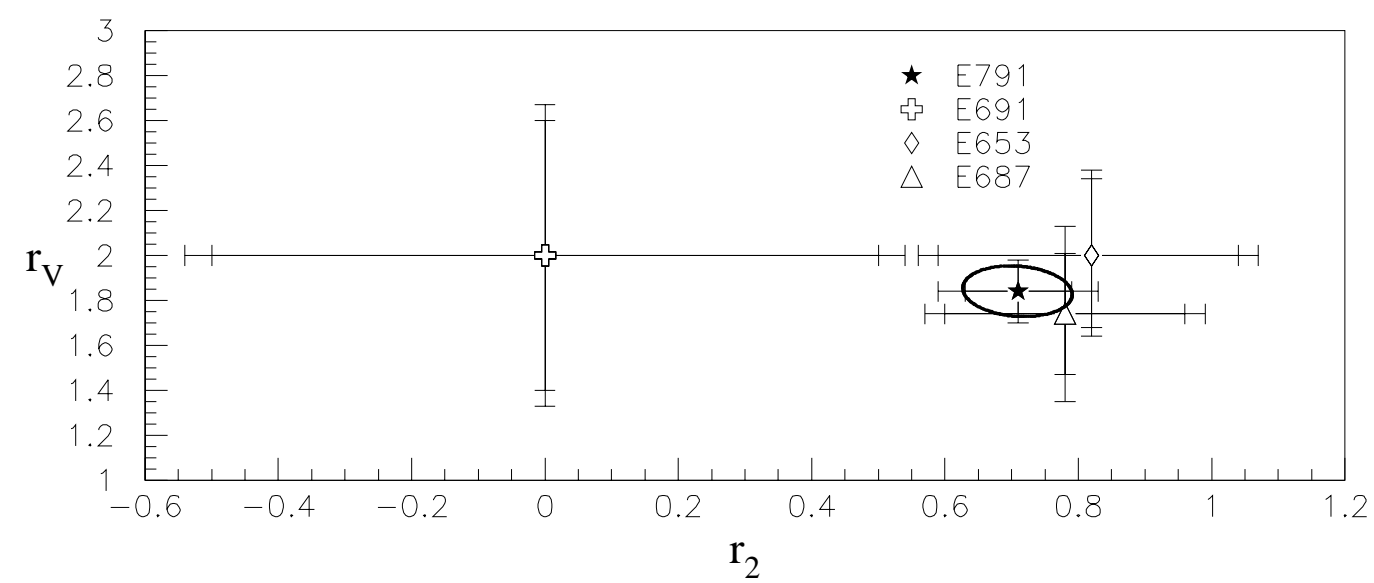

Figure 8.1: Experimental measurements of $r_{2}$ and $r_{V}$ by E791 and other groups. For each measurement the smaller error bar is the statistical error. The larger is the statistical and systematic errors added in quadrature. 
rate expressed as a function of the form factors measured at $q^{2}=0$ :

$$
\begin{aligned}
,\left(D^{+} \rightarrow \bar{K}^{* 0} e^{+} \nu_{e}\right)= & 1.915 \times 10^{11} s^{-1}\left|V_{c s}\right|^{2}\left[A_{1}^{2}(0)+0.045 A_{2}^{2}(0)\right. \\
& \left.-0.33 A_{1}(0) A_{2}(0)+0.017 V^{2}(0)\right] .
\end{aligned}
$$

This calculation assumes the nearest-pole-dominance model of $q^{2}$ dependence, with $m_{A}=2.5 \mathrm{GeV}$ and $m_{V}=2.1 \mathrm{GeV}$. Plugging in the latest PDG values for , $\left(D^{+} \rightarrow\right.$ $\left.\bar{K}^{* 0} e^{+} \nu_{e}\right)=(4.54 \pm 0.38) \times 10^{10} s^{-1}$ and $V_{c s}=0.975$ [4], and rearranging the terms we get the following expression for $A_{1}(0)$ as a function of $r_{2}$ and $r_{V}$ :

$$
A_{1}(0)=\left[\frac{4.60 \times 10^{10}}{1.915 \times 10^{11}(0.975)^{2}\left(1+0.045 r_{2}^{2}-0.33 r_{2}+0.017 r_{V}^{2}\right)}\right]^{1 / 2} .
$$

Thus it is possible to solve for $A_{1}(0), A_{2}(0)$, and $V(0)$. Using the nearest-poledominance model for the $q^{2}$ dependence, we also derive the values of the form factors at $q^{2}=q_{\max }^{2}$. The E791 measurements of the form factors at $q^{2}=0$ and $q^{2}=q_{\max }^{2}$ are listed in Table 8.2.

The method outlined above assumes the following form for the $M_{K \pi}$ dependence of the differential decay rate:

$$
\frac{d,}{d M_{K \pi}}=\delta\left(M_{K \pi}-M_{K^{* 0}}\right) .
$$

A more accurate form for the $M_{K \pi}$ dependence is given by a Breit-Wigner distribution, as seen in Eq. 1.7. We use a numerical integration code [41] with the input of $\mathrm{B}\left(D^{+} \rightarrow\right.$ $\left.\bar{K}^{* 0} e^{+} \nu_{e}\right)$ and the $D^{+}$lifetime to calculate the form factors. This code also properly accounts for the correlation between the errors on the form-factor ratios. The results for $A_{1}, A_{2}$, and $V$ at $q^{2}=0$ and at $q^{2}=q_{\max }^{2}$ are given in Table 8.2.

These results may be compared to the theoretical predictions in Table 8.3. E791 measurements agree fairly well with some of the Lattice Gauge calculations [21, 22]. The lowest-order HQET predictions based on Eqs. 1.13 and 1.14 are listed along with the predictions of ISGW2 [7], a quark model calculation which is based on heavy quark symmetry. The naive HQET prediction does not agree with experimental results. It 


\begin{tabular}{|l|c|c|}
\hline Form Factor & $q^{2}=0$ & $q^{2}=q_{\max }^{2}$ \\
\hline \multicolumn{3}{|c|}{$\frac{d \Gamma}{d M_{K \pi}}=\delta\left(M_{K \pi}-M_{K^{* 0}}\right)$} \\
\hline$A_{1}\left(q^{2}\right)$ & $0.54 \pm 0.02 \pm 0.01$ & $0.64 \pm 0.03 \pm 0.01$ \\
$A_{2}\left(q^{2}\right)$ & $0.39 \pm 0.05 \pm 0.05$ & $0.45 \pm 0.06 \pm 0.06$ \\
$V\left(q^{2}\right)$ & $1.00 \pm 0.07 \pm 0.04$ & $1.27 \pm 0.09 \pm 0.05$ \\
\hline \multicolumn{3}{|c|}{$\frac{d \Gamma}{d M_{K \pi}}=$ Breit-Wigner } \\
\hline$A_{1}\left(q^{2}\right)$ & $0.58 \pm 0.03 \pm 0.01$ & $0.68 \pm 0.04 \pm 0.01$ \\
$A_{2}\left(q^{2}\right)$ & $0.41 \pm 0.04 \pm 0.05$ & $0.48 \pm 0.05 \pm 0.06$ \\
$V\left(q^{2}\right)$ & $1.06 \pm 0.08 \pm 0.05$ & $1.35 \pm 0.10 \pm 0.06$ \\
\hline
\end{tabular}

Table 8.2: E791 results for the form factors $A_{1}, A_{2}$, and $V$, at $q^{2}=0$ and $q^{2}=q_{\text {max }}^{2}$, calculated for two different assumptions for shape of the $\bar{K}^{* 0}$ resonance.

\begin{tabular}{|l|c|c|c|}
\hline Group & $A_{1}(0)$ & $A_{2}(0)$ & $V(0)$ \\
\hline E791 & $0.58 \pm 0.03 \pm 0.01$ & $0.41 \pm 0.04 \pm 0.05$ & $1.06 \pm 0.08 \pm 0.05$ \\
\hline APE [22] & $0.67 \pm 0.11$ & $0.49 \pm 0.34$ & $1.08 \pm 0.22$ \\
Wuppertal [25] & $0.61_{-0.09}^{+0.11}$ & $0.83_{-0.22}^{+0.23}$ & $1.34_{-0.28}^{+0.31}$ \\
UKQCD [23] & $0.70_{-0.10}^{+0.07}$ & $0.66_{-0.15}^{+0.10}$ & $1.01_{-0.13}^{+0.30}$ \\
ELC [21] & $0.64 \pm 0.16$ & $0.41 \pm 0.28 \pm 0.04$ & $0.86 \pm 0.24$ \\
\hline \hline & $A_{1}\left(q_{\max }^{2}\right)$ & $A_{2}\left(q_{\max }^{2}\right)$ & $V\left(q_{\max }^{2}\right)$ \\
\hline E791 & $0.68 \pm 0.04 \pm 0.01$ & $0.48 \pm 0.05 \pm 0.06$ & $1.35 \pm 0.10 \pm 0.06$ \\
\hline HQET & 0.94 & 1.07 & 1.07 \\
ISGW2 [7] & 0.70 & 0.94 & 1.52 \\
\hline
\end{tabular}

Table 8.3: Comparison of E791 results for the form factors $A_{1}, A_{2}$, and $V$ with theoretical predictions. 
is not expected to since the $s$ quark cannot be treated as heavy. ISGW2 incorporates the constrains imposed by heavy quark symmetry and $1 / M_{Q}$ corrections due to the breaking of heavy quark symmetry, yet its predictions are also not in good agreement with the experimental results, especially for $A_{2}\left(q_{\text {max }}^{2}\right)$.

With a multidimensional unbinned fit there is no clear "goodness of fit" parameter such as $\chi^{2}$ for binned fits. A useful way of evaluating the goodness of fit is to overlay the one-dimensional projections of the best fit on top of the kinematic variable distributions. Figure 8.2 shows the difference between right-sign and wrong-sign distributions of the kinematic variables, overlaid by Monte Carlo events that have been weighted according to the best fit values of the form-factor ratios $\left(r_{2}=0.71\right.$ and $\left.r_{V}=1.84\right)$. Also a $\chi^{2} / d o f$ and a $\chi^{2}$ probability is calculated and shown on each distribution. The number of degrees of freedom for each plot is given by the number of bins (20) - number of fit parameters (1, for overall normalization). It appears that the results of the fit are sensible. For comparison, similar distributions with the generated form factor ratios $\left(r_{2}=0.82\right.$ and $\left.r_{V}=2.00\right)$ are plotted in Figure 8.3. The $\chi^{2}$ probabilities for $\cos \theta_{l}$ and $q^{2} / q_{\max }^{2}$ in Figure 8.3 are somewhat worse than those in Figure 8.2, suggesting that the E653 measurements for the form factor ratios are less appropriate for E791 data.

It can also be informative to look at the distributions that exhibit correlations between the kinematic variables. Three such distributions are plotted in Figure 8.4. The left plots are of $\cos \theta_{V}$ in two regions of $q^{2} / q_{\max }^{2}$. A pronounced $\cos ^{2} \theta_{V}$ distribution from the $H_{0}^{2}$ term is seen at low $q^{2} / q_{\max }^{2}$. This distribution does not depend strongly on the values of the form-factor ratios (See Figure A.1). In the middle are plots of $\cos \theta_{l}$ in two regions of $q^{2} / q_{\max }^{2}$. The shape of this distribution at higher values of $q^{2} / q_{\max }^{2}$ depends strongly on $r_{V}$. The observed distribution implies that $r_{V}$ is significantly different from 0 . The right plots are of $\chi$ in two regions of $\cos \theta_{V}$. This distribution is also affected strongly by the value for $r_{V}$. All these distributions (along with the other three possible combinations of the kinematic variables) are discussed in greater detail in Appendix A. The $\chi^{2}$ probability is calculated for each distribution. As can be seen from the plots, the Monte Carlo properly accounts for the correlations between the kinematic variables. 

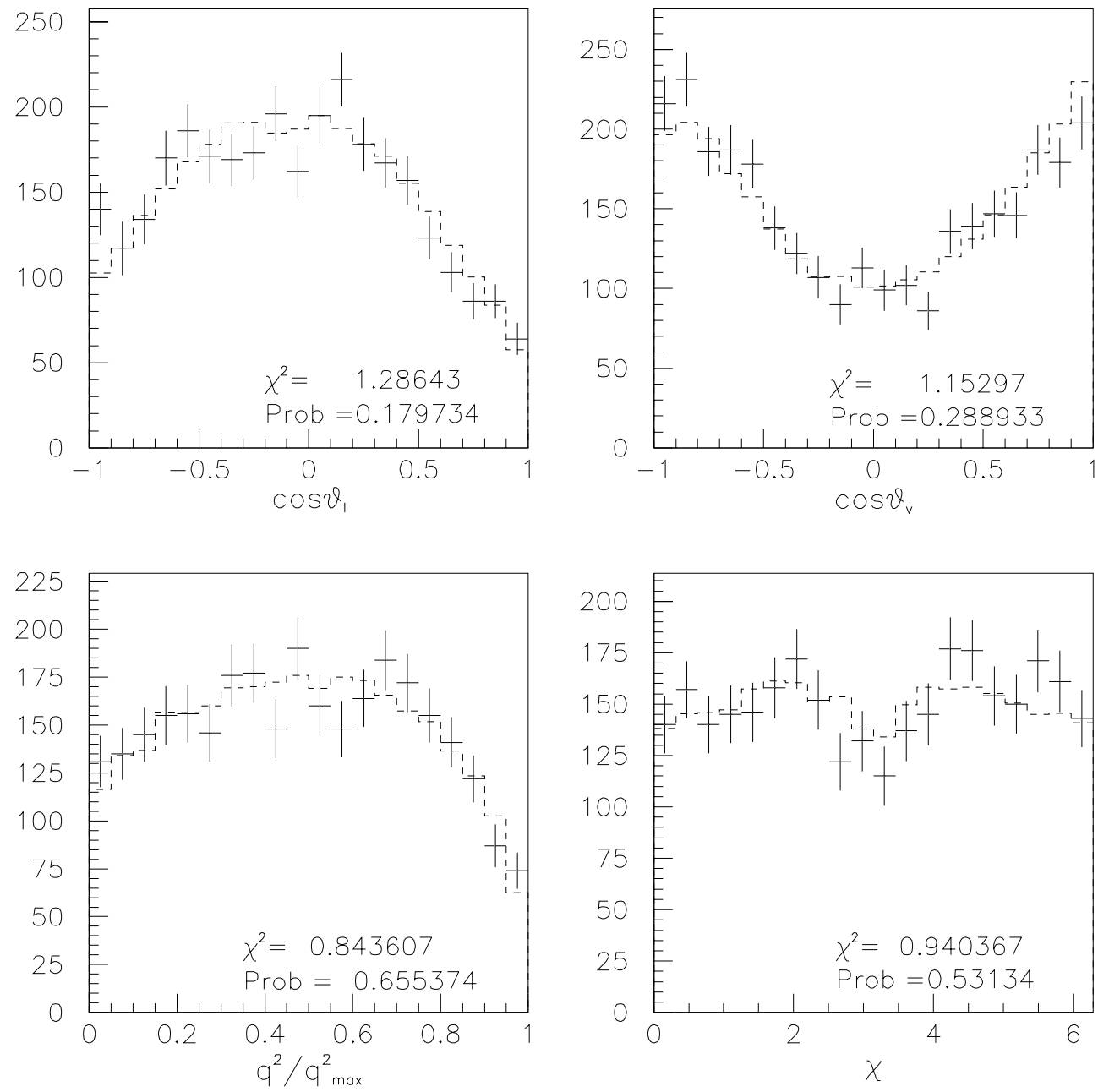

Figure 8.2: Background subtracted data distributions (crosses) overlaid with Monte Carlo (dashed histogram) with best fit values for the form factor ratios $\left(r_{2}=0.71\right.$, $\left.r_{V}=1.84\right)$. The $\chi^{2}$ per degree of freedom and the $\chi^{2}$ probability are shown on each plot. 

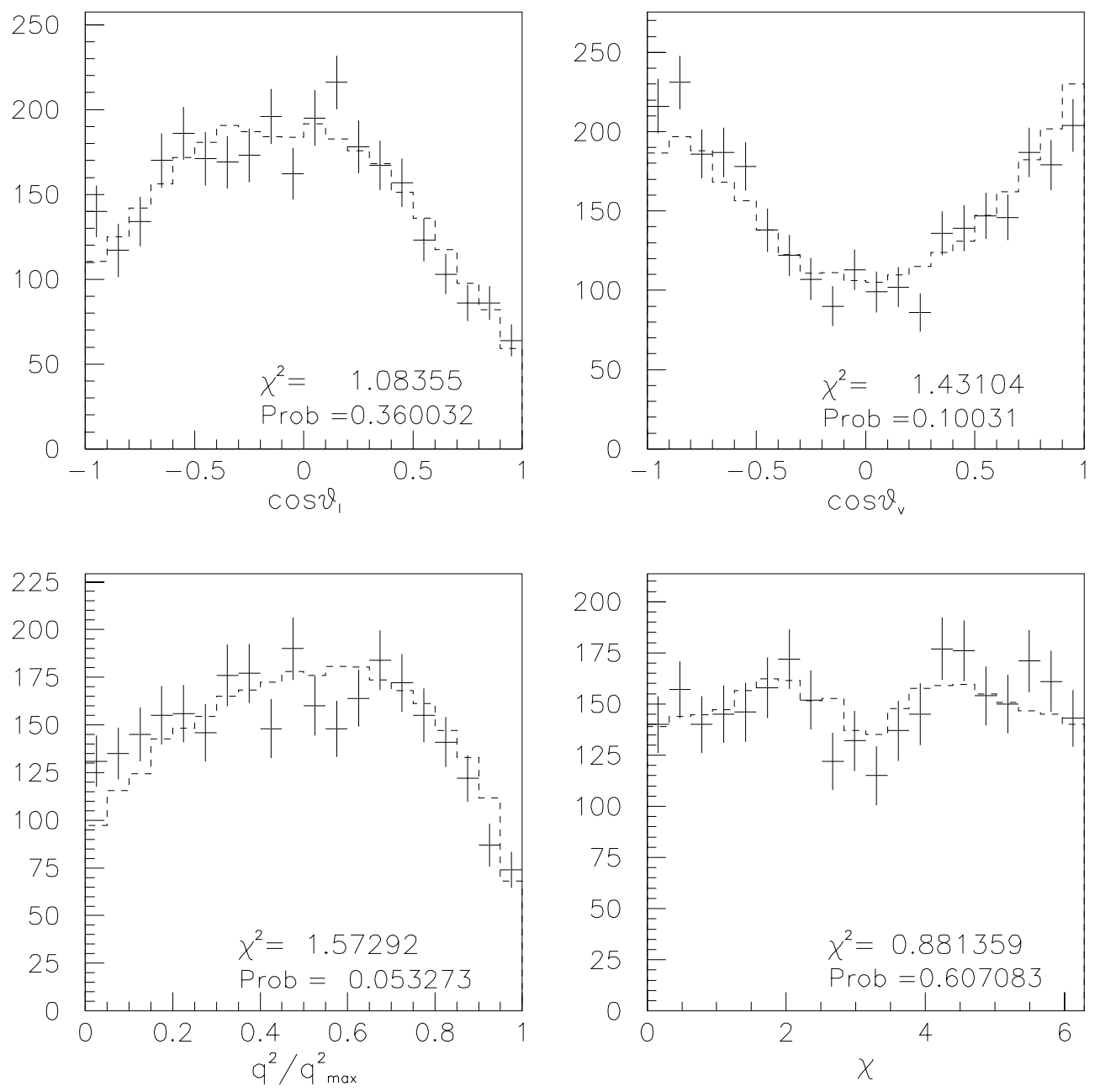

Figure 8.3: Background subtracted data distributions (crosses) overlaid with Monte Carlo (dashed histogram) with E653 values for the form factor ratios $\left(r_{2}=0.82, r_{V}=\right.$ 2.00). The $\chi^{2}$ per degree of freedom and the $\chi^{2}$ probability are shown on each plot. 
(a)

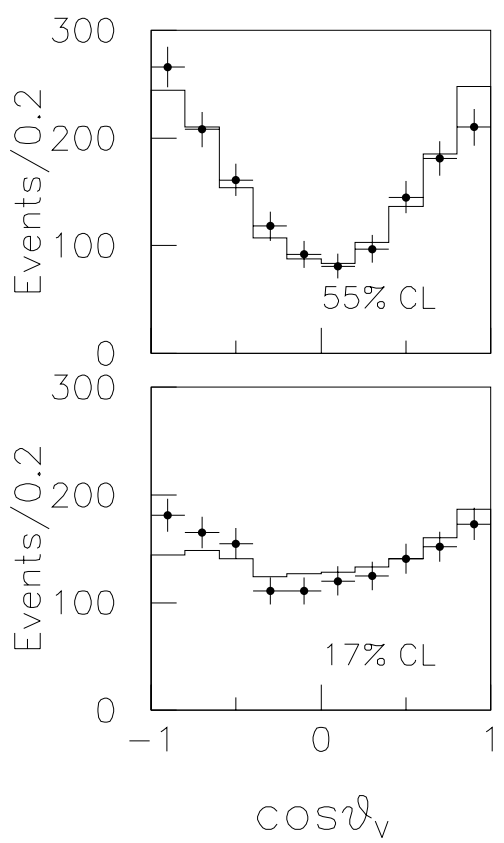

(b)

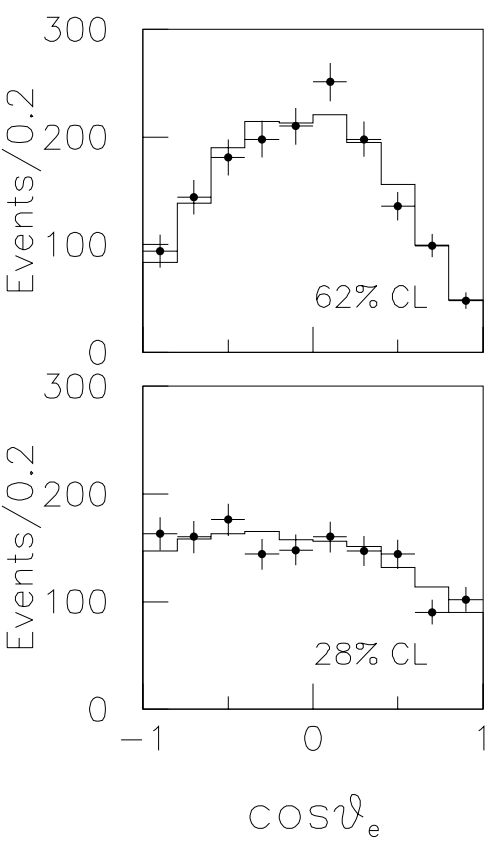

(c)

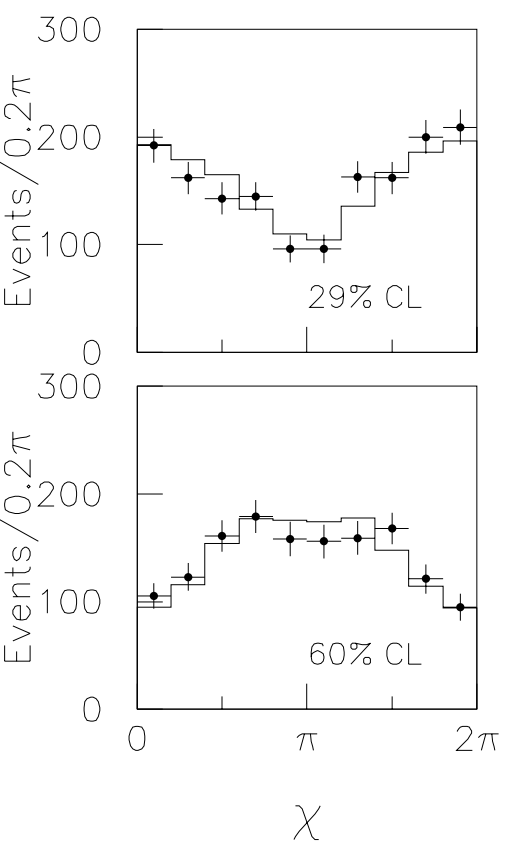

Figure 8.4: Background subtracted data distributions (black dots) overlaid with Monte Carlo (dashed histogram) with best fit values for the form factor ratios for (a) $\cos \theta_{V}$ for $q^{2} / q_{\text {max }}^{2} \leq 0.5$ (top) and $q^{2} / q_{\text {max }}^{2}>0.5$ (bottom), (b) $\cos \theta_{e}$ for $q^{2} / q_{\text {max }}^{2} \leq 0.5$ (top) and $q^{2} / q_{\max }^{2}>0.5$ (bottom), (c) $\chi$ for $\cos \theta_{V} \leq 0$ (top) and $\cos \theta_{V}>0$ (bottom). The $\chi^{2}$ probability is shown on each figure. 


\subsection{Three-Parameter Fit}

We also performed a three-parameter fit in which we fit for the slope of the $q^{2}$ dependence, $\rho$, as well as the form factor ratios $r_{2}$ and $r_{V}$, using

$$
F\left(q^{2}\right)=F(0)\left(1+\rho q^{2}\right)
$$

The ratio of the slopes for the vector and axial-vector form factors is fixed such that

$$
\rho_{V}=\rho_{A}\left(\frac{m_{\text {pole }}^{A}}{m_{\text {pole }}^{V}}\right)^{2} .
$$

$\rho_{A}$ is a free parameter in the fit along with $r_{2}$ and $r_{V}$. The results are $r_{2}=0.73_{-0.15}^{+0.14}$, $r_{V}=2.01 \pm 0.11$, and $\rho_{A}=0.11_{-0.09}^{+0.10} \mathrm{GeV}^{-2}$. The total error matrix is:

\begin{tabular}{c|ccc} 
& $r_{2}$ & $r_{V}$ & $\rho_{A}$ \\
\hline$r_{2}$ & 1.000 & 0.083 & -0.849 \\
$r_{V}$ & 0.083 & 1.000 & -0.174 \\
$\rho_{A}$ & -0.849 & -0.174 & 1.000
\end{tabular}

Although the Monte Carlo was not generated with the $q^{2}$ dependence described by Eq. 1.6, the linear form for the $q^{2}$ dependence is effectively identical to it in the $q^{2}$ range examined. We measure the systematic bias due to the fitting technique by using twenty small Monte Carlo samples, as in the two-parameter fit. The values of the biases and the corrected results for the three fit parameters are listed in Table 8.4. The measured value for $\rho_{A}$ is smaller that one would naively expect, namely $\rho=$ $1 / m_{\text {pole }}^{2}=0.16 \mathrm{GeV}^{-2}$, but it is within two standard deviations. $r_{2}$ and $\rho_{A}$ are very strongly anti-correlated which leads to large uncertainties on the values for the two parameters. The value for $r_{2}$ is different from the result in the standard two-parameter fit, although the statistical uncertainty is greater. The result for $r_{V}$ is not significantly changed from that found with the standard fit. 


\begin{tabular}{|l|c|c|c|}
\hline Parameter & Fit Value & Bias & Corrected Value \\
\hline$r_{2}$ & $0.73_{-0.15}^{+0.14}$ & -0.25 & $0.98_{-0.15}^{+0.14}$ \\
$r_{V}$ & $2.01 \pm 0.11$ & +0.13 & $1.88 \pm 0.11$ \\
$\rho_{A}\left(\mathrm{GeV}^{-2}\right)$ & $0.11_{-0.09}^{+0.10}$ & +0.17 & $-0.06_{-0.09}^{+0.10}$ \\
\hline
\end{tabular}

Table 8.4: The results of the fit for the parameters $r_{2}, r_{V}$, and slope, the systematic bias due to the fitting technique and the corrected values.

\subsection{Conclusion}

We measure the form-factor ratios, $r_{2}=0.71 \pm 0.08 \pm 0.09$ and $r_{V}=1.84 \pm 0.11 \pm 0.08$, in the semileptonic decay $D^{+} \rightarrow \bar{K}^{* 0} e^{+} \nu_{e}$ from data collected by Fermilab experiment E791. This is the most precise measurement to date. Also, the systematic errors are significantly smaller than those reported by previous experiments. Several Lattice Gauge calculations are in good agreement with these results. On the other hand, some predictions from quark model calculations are not. In addition, we make the first measurement of the slope of $q^{2}$ dependence in $D^{+} \rightarrow \bar{K}^{* 0} e^{+} \nu_{e}$ decay. The values extracted from a three-parameter fit are $r_{2}=0.98_{-0.15}^{+0.14}, r_{V}=1.88 \pm 0.11$, and $\rho_{A}=$ $-0.06_{-0.09}^{+0.10} \mathrm{GeV}^{-2}$. 


\section{Appendix A}

\section{Kinematic Variable Distributions}

Using the commercial software Mathematica [42] we study the distribution of the differential decay rate given by Eq. 1.7 in the four-dimensional kinematic variable space for different values of the form-factor ratios. The test values were chosen, such that we could show how the decay rate distribution is affected when each of the formfactor ratios is changed. We compare the distributions at $r_{2}=r_{V}=0$ to $r_{2}=1$ and $r_{V}=2$, which are approximately the values measured by this analysis, as well to cases where $r_{2}=1$ or $r_{V}=2$, and the other ratio is kept at 0 .

Since it is not possible to view the entire four-dimensional distribution, we instead integrate over two of the variables and plot the decay rate distribution in the remaining two of the variables. A simplified version of Eq. 1.7 is used where the Breit-Wigner describing the distribution of $M_{K \pi}$ is instead replaced by a $\delta$-function, $M_{K \pi}=M_{K^{*}}$ and integrated over $M_{K \pi}^{2}$. The helicity amplitudes given by Eq. 1.8 are assumed to be real. In addition, the overall constants which do not influence the shape of the distributions are dropped. The expression for the differential decay rate then becomes

$$
\begin{aligned}
\frac{d,}{d q^{2} d \cos \theta_{V} d \cos \theta_{l} d \chi} & =p_{K} q^{2} \times\left\{\left(1+\cos \theta_{l}\right)^{2}\left[H_{+}\left(q^{2}\right)\right]^{2} \sin ^{2} \theta_{V}\right. \\
& +\left(1-\cos \theta_{l}\right)^{2}\left[H_{-}\left(q^{2}\right)\right]^{2} \sin ^{2} \theta_{V} \\
& +4 \sin ^{2} \theta_{l} \cos ^{2} \theta_{V}\left[H_{0}\left(q^{2}\right)\right]^{2} \\
& -2 \sin ^{2} \theta_{l} \sin ^{2} \theta_{V} \cos 2 \chi H_{+} H_{-}
\end{aligned}
$$




$$
\begin{aligned}
& -4 \sin \theta_{l}\left(1+\cos \theta_{l}\right) \sin \theta_{V} \cos \theta_{V} \cos \chi H_{+} H_{0} \\
& \left.+4 \sin \theta_{l}\left(1-\cos \theta_{l}\right) \sin \theta_{V} \cos \theta_{V} \cos \chi H_{-} H_{0}\right\}
\end{aligned}
$$

where $p_{K}$ depends on $q^{2}$ and is given by

$$
p_{K}=\frac{\sqrt{\left(M_{D}^{2}-M_{K^{*}}^{2}-q^{2}\right)^{2}-4 M_{K^{*}}^{2} q^{2}}}{2 M_{D}} .
$$

Thus there are six terms in the equation, not all of which contribute to each twodimensional distribution. Each distribution is shown and discussed in greater detail in the following sections.

\section{A.1 $\cos \theta_{V} \mathbf{v} \cdot q^{2}$}

Only the first three terms of Eq. A.1 contribute to this distribution, since

$$
\int_{0}^{2 \pi} \cos \chi d \chi=\int_{0}^{2 \pi} \cos 2 \chi d \chi=0 .
$$

The integration over $\chi$ for the remaining terms simply gives an overall factor of $2 \pi$. The distributions shown in Figure A.1 represent the sum

$$
p_{K} q^{2}\left\{\sin ^{2} \theta_{V}\left(H_{+}^{2}+H_{-}^{2}\right)+2 \cos ^{2} \theta_{V} H_{0}^{2}\right\}
$$

following an integration over $\cos \theta_{l}{ }^{1}$ The contributions from the first two terms are small in comparison to the third. The differential decay rate, $\frac{d \Gamma}{d \cos \theta_{V} d q^{2}}$, is plotted for four sets of values of $r_{2}$ and $r_{V}$. As can be seen from the plot, changing $r_{V}$ has little effect on the distribution, since $r_{V}$ only contributes to the $H_{ \pm}^{2}$ terms. A clear $\cos ^{2} \theta_{V}$ distribution from the $H_{0}^{2}$ term is evident in each plot at low $q^{2}$. At higher values of $q^{2}$ the small $\sin ^{2} \theta_{V}$ contribution from the $H_{ \pm}^{2}$ terms is evident.

\footnotetext{
${ }^{1}$ Overall constants have been dropped.
} 

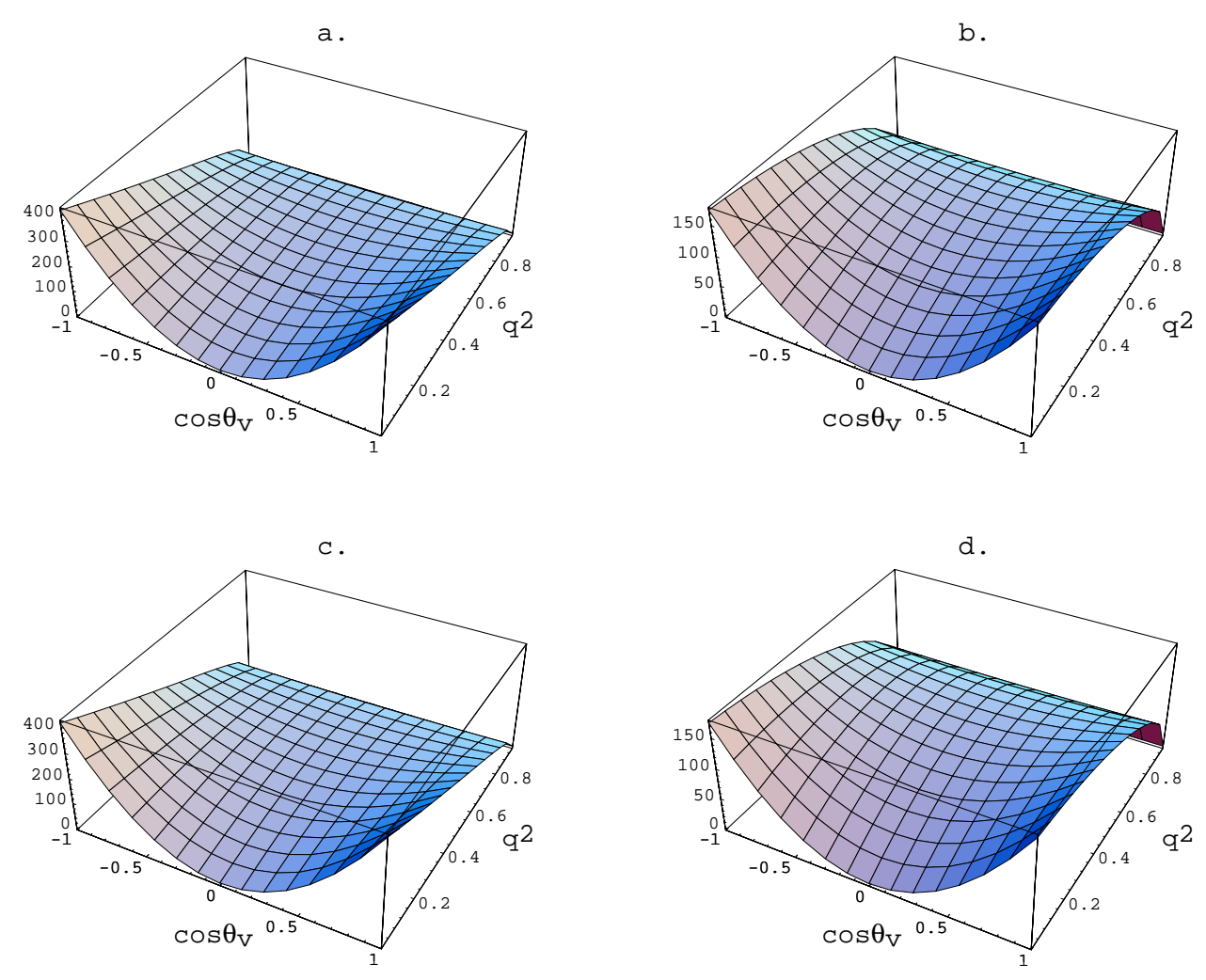

Figure A.1: The differential decay rate distribution in the space spanned by $\cos \theta_{V}$ and $q^{2}$ for four sets of form-factor ratios: a. $r_{2}=0, r_{V}=0$; b. $r_{2}=1, r_{V}=0$; c. $r_{2}=0$, $r_{V}=2$; and d. $r_{2}=1, r_{V}=2$. 


\section{A.2 $\cos \theta_{l} \mathbf{v} \cdot q^{2}$}

As in the previous section, only the first three terms of Eq. A.1 contribute to this distribution. Again the integration over $\chi$ adds only an overall factor of $2 \pi$. The integration over $\cos \theta_{V}$ gives the following expression

$$
p_{K} q^{2}\left\{\left(1+\cos \theta_{l}\right)^{2} H_{+}^{2}+\left(1-\cos \theta_{l}\right)^{2} H_{-}^{2}+2 \sin ^{2} \theta_{l} H_{0}^{2}\right\}
$$

which is plotted in Figure A.2 for four sets of values for the form-factor ratios. At low values of $r_{2}$ and $r_{V}$, the $H_{0}^{2}$ term is the more dominant. We see the $\sin ^{2} \theta_{l}$ distribution at low $q^{2}$. The $H_{ \pm}^{2}$ terms contribute more as $r_{2}$ increases. The $H_{-}^{2}$ term becomes the most dominant at increasing $r_{V}$ as can be seen from the $\left(1-\cos \theta_{l}\right)^{2}$ distribution at intermediate values of $q^{2}$ in plot d. of Figure A.2.

\section{A.3 $\chi \mathbf{v} \cdot q^{2}$}

The first four terms of Eq. A.1 contribute to this distribution, since

$$
\int_{-1}^{1} \cos \theta_{V} \sqrt{1-\cos ^{2} \theta_{V}} d \cos \theta_{V}=0
$$

The integrations over $\cos \theta_{V}$ and $\cos \theta_{l}$ for the remaining terms give the following expression

$$
p_{K} q^{2}\left\{H_{+}^{2}+H_{-}^{2}+H_{0}^{2}-H_{+} H_{-} \cos 2 \chi\right\}
$$

which is plotted in Figure A.3 for four sets of values for the form-factor ratios. Only the $H_{+} H_{-}$cross term has any $\chi$ dependence. The $-\cos 2 \chi$ distribution is evident in each plot at intermediate values of $q^{2}$. These distributions give a clear view of the $q^{2}$ dependence of each helicity amplitude and its dependence on the form factor ratios. The $H_{0}^{2}$ term is the only one that does not equal 0 at $q^{2}=0$; thus it dominates at low $q^{2}$. When $r_{V}=0, H_{+}^{2}=H_{-}^{2}=H_{+} H_{-}$. As $r_{V}$ increases, the $H_{-}^{2}$ term becomes more dominant, as can be seen from Eq. 1.8. When $r_{2}$ increases, the $H_{0}^{2}$ term decreases, and becomes comparable in size to the other terms. 

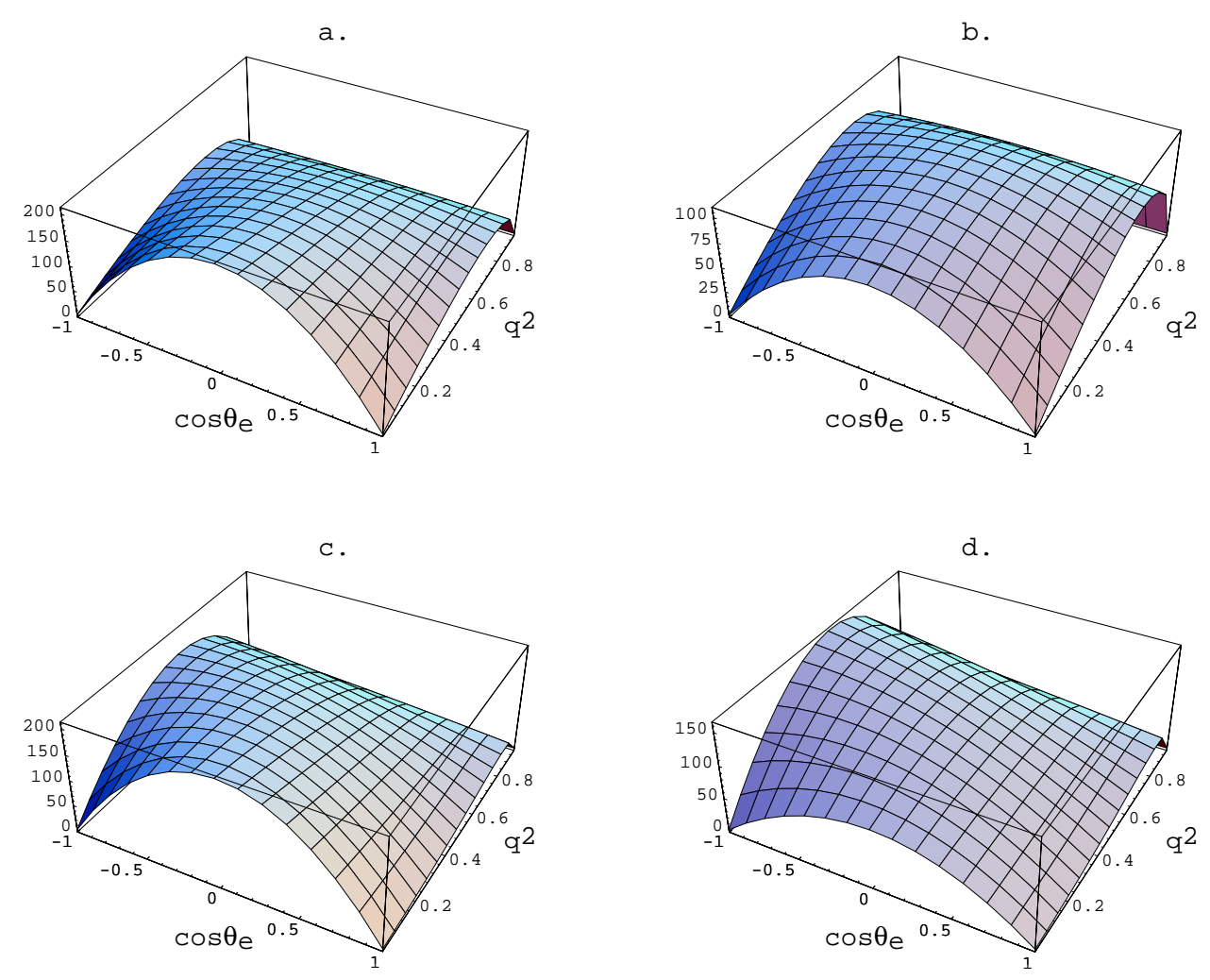

Figure A.2: The differential decay rate distribution in the space spanned by $\cos \theta_{l}$ and $q^{2}$ for four sets of form-factor ratios: a. $r_{2}=0, r_{V}=0$; b. $r_{2}=1, r_{V}=0 ;$ c. $r_{2}=0$, $r_{V}=2$; and d. $r_{2}=1, r_{V}=2$. 

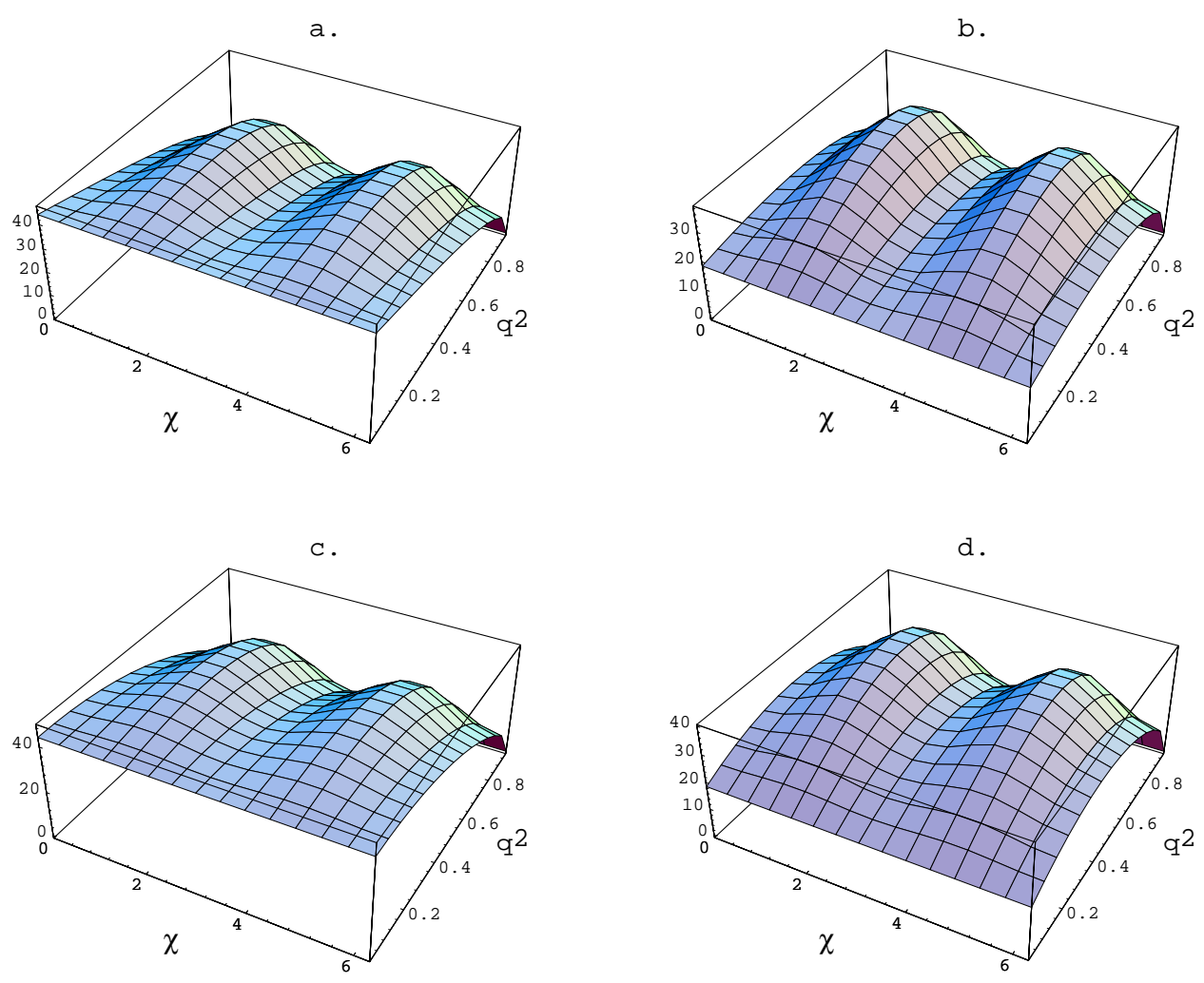

Figure A.3: The differential decay rate distribution in the space spanned by $\chi$ and $q^{2}$ for four sets of form-factor ratios: a. $r_{2}=0 ., r_{V}=0$; b. $r_{2}=1, r_{V}=0 ;$ c. $r_{2}=0, r_{V}=2$; and d. $r_{2}=1, r_{V}=2$. 

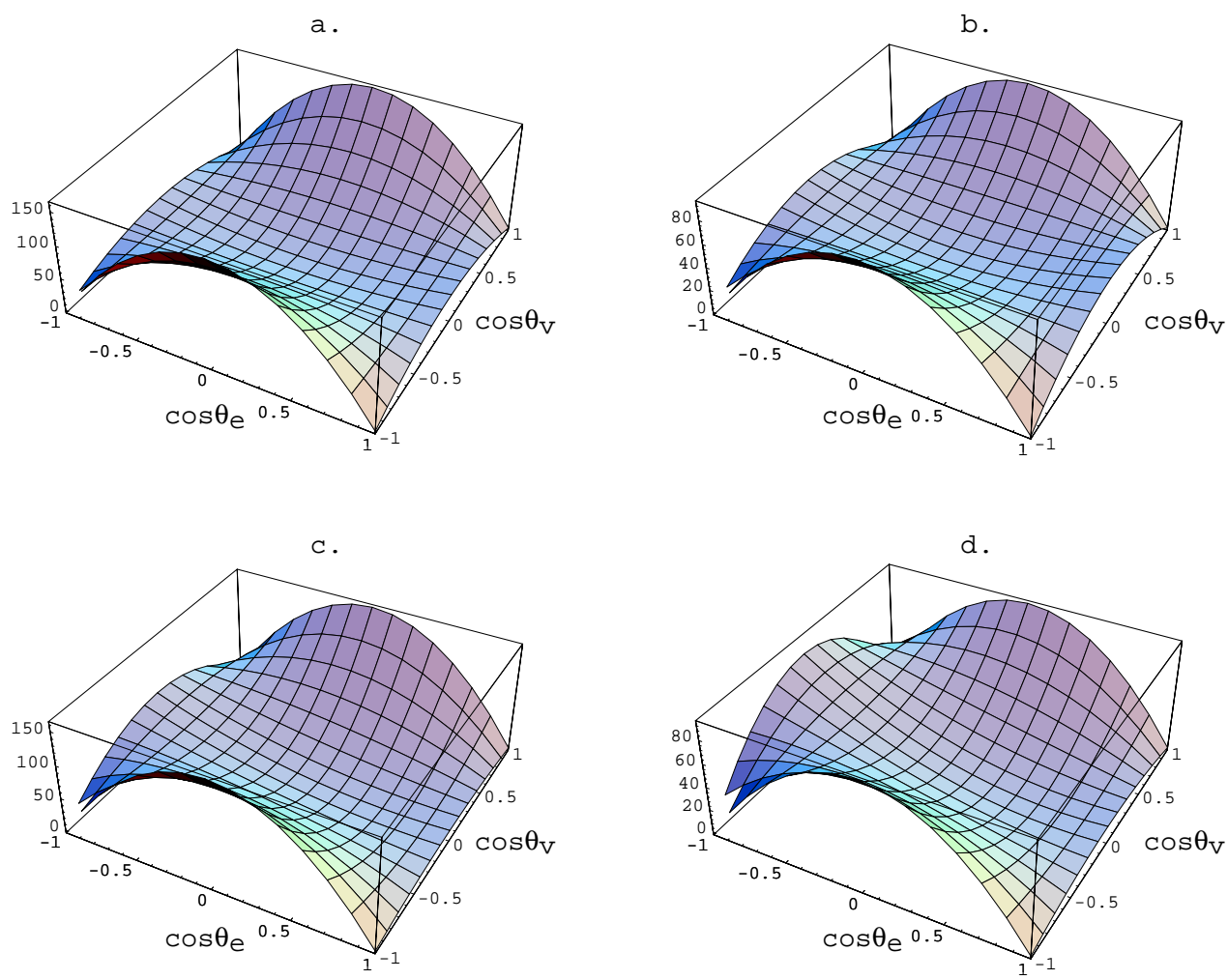

Figure A.4: The differential decay rate distribution in the space spanned by $\cos \theta_{l}$ and $\cos \theta_{V}$ for four sets of form-factor ratios: a. $r_{2}=0, r_{V}=0 ;$ b. $r_{2}=1, r_{V}=0 ;$ c. $r_{2}=0, r_{V}=2$; and d. $r_{2}=1, r_{V}=2$.

\section{A.4 $\cos \theta_{l} \mathbf{v} \cdot \cos \theta_{V}$}

The full power of Mathematica is necessary to integrate over $q^{2}$. Only the first three terms of Eq. A.1 contribute to $\frac{d \Gamma}{d \cos \theta_{l} d \cos \theta_{V}}$. The cross terms vanish due to the integration over $\chi$ as shown in Eq. A.3. Figure A.4 shows the distributions for four values of the form-factor ratios. Some of the features discussed in the previous sections are also seen in these plots. At $r_{2}=r_{V}=0$, the third term is dominant as can be seen from the strong $\cos ^{2} \theta_{V}$ distribution. As $r_{2}$ increases this term gets smaller. As $r_{V}$ increases the second term, along with its $\left(1-\cos \theta_{l}\right)^{2}$ distribution, becomes more important, and the first term becomes less significant. 

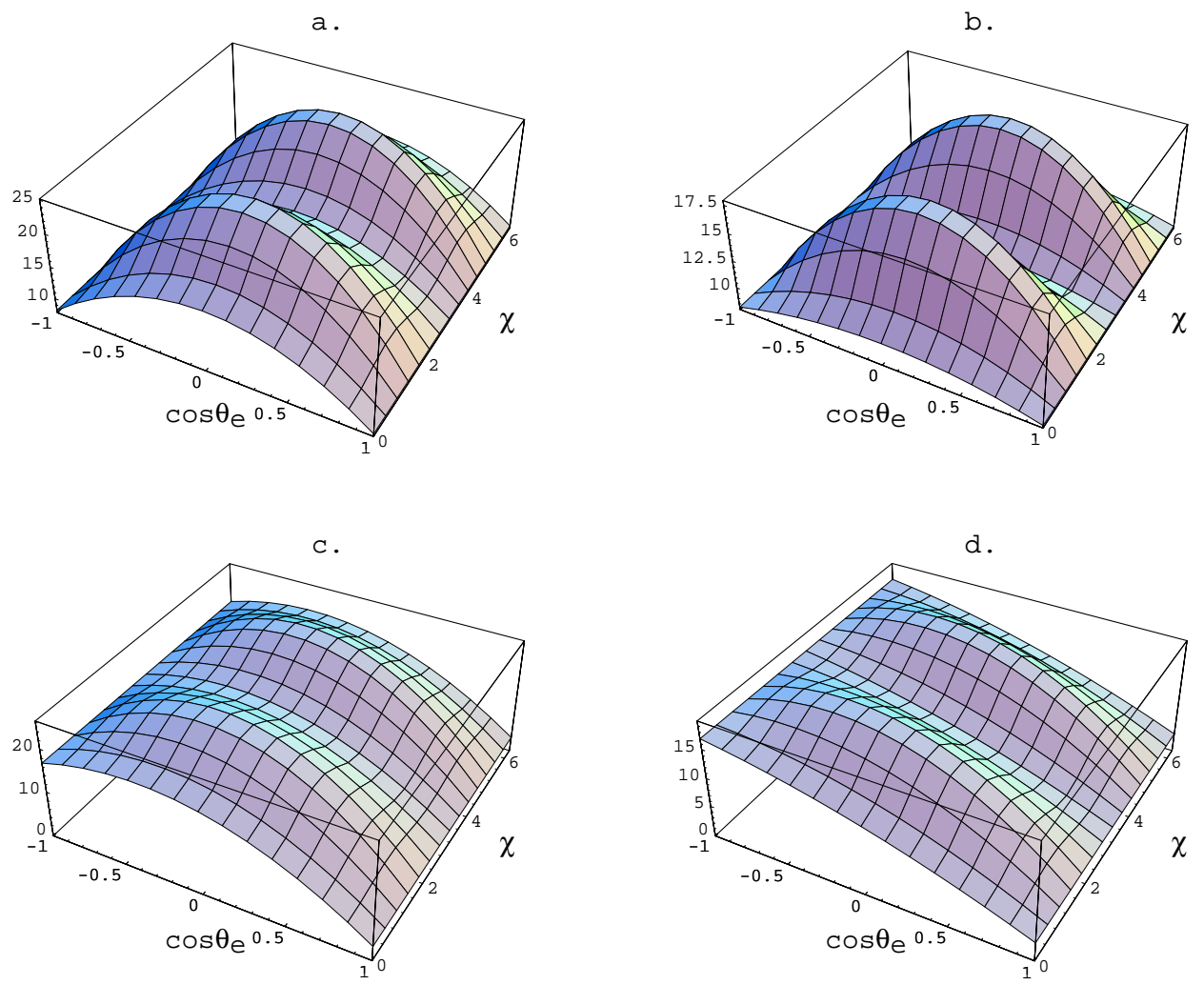

Figure A.5: The differential decay rate distribution in the space spanned by $\cos \theta_{l}$ and $\chi$ for four sets of form-factor ratios: a. $r_{2}=0, r_{V}=0$; b. $r_{2}=1, r_{V}=0$; c. $r_{2}=0$, $r_{V}=2 ;$ and d. $r_{2}=1, r_{V}=2$.

\section{A.5 $\cos \theta_{l} \mathbf{v} \cdot \chi$}

The first four terms of Eq. A.1 contribute to this distribution. The last two terms vanish due to the integration over $\cos \theta_{V}$ as shown in Eq. A.6. Figure A.5 shows the distributions for four sets of form-factor ratios. Only the fourth term of the equation depends on $\chi$. The $-\cos 2 \chi$ distribution is evident in each plot. At $r_{V}=0$, the first two terms are small. As $r_{V}$ increases the second term becomes more significant than the first. As $r_{2}$ increases the third term decreases in importance. Therefore less of the $\sin ^{2} \theta_{l}$ distribution is seen. 

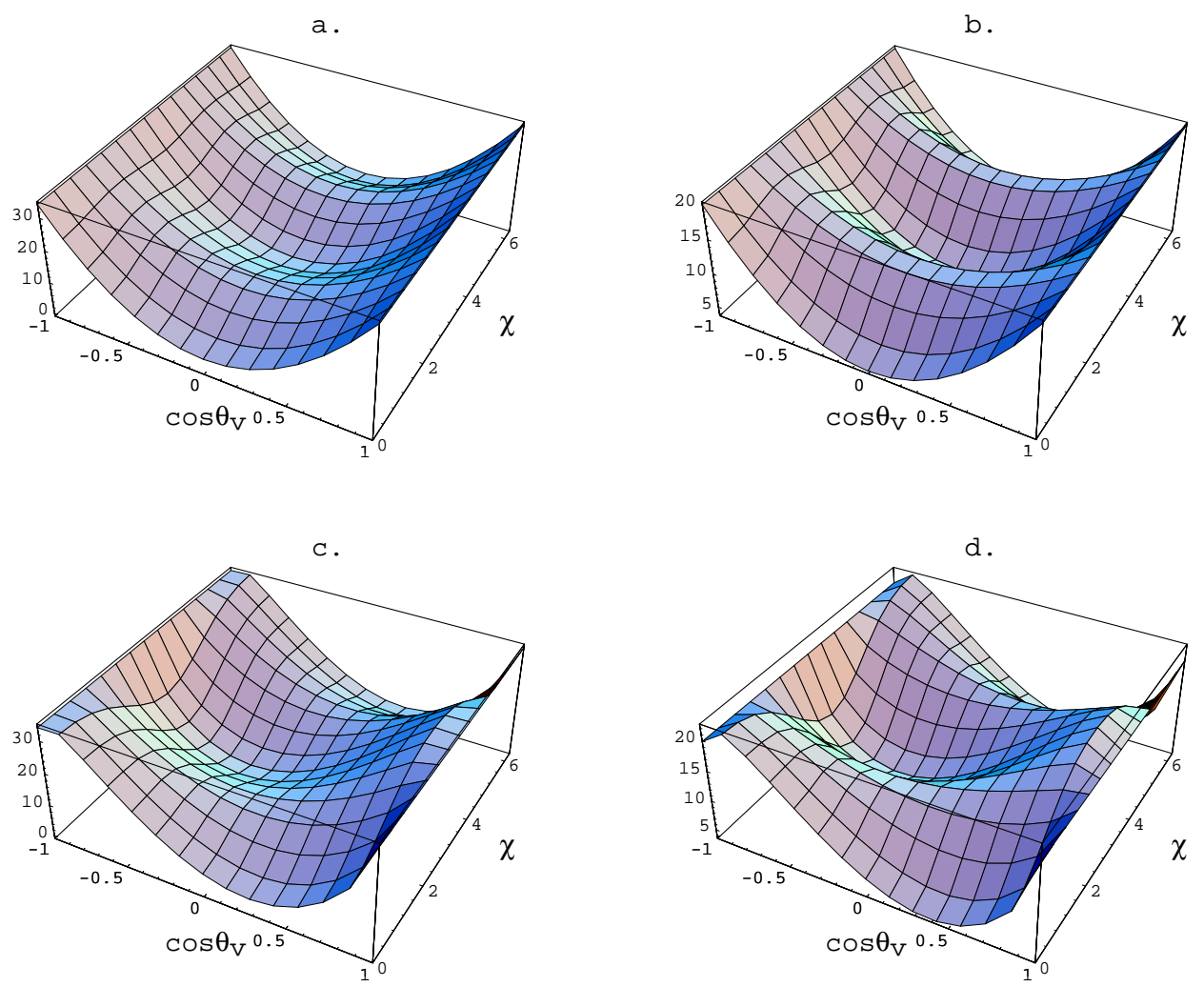

Figure A.6: The differential decay rate distribution in the space spanned by $\cos \theta_{V}$ and $\chi$ for four sets of form-factor ratios: a. $r_{2}=0, r_{V}=0 ;$ b. $r_{2}=1, r_{V}=0 ;$ c. $r_{2}=0$, $r_{V}=2 ;$ and d. $r_{2}=1, r_{V}=2$.

\section{A.6 $\cos \theta_{V} \mathbf{v} \cdot \chi$}

This last of the six possible two-variable combinations is the most complicated to generate. This is the only distribution where all six terms in Eq. A.1 contribute. Integration over $q^{2}$ for the $H_{+} H_{0}$ and $H_{-} H_{0}$ cross terms was done numerically by Mathematica. Plots of the two-dimensional distribution for the four sets of formfactor ratios, are shown in Figure A.6. Now three terms depend on $\chi$. The $-\cos 2 \chi$ dependence is seen in every plot near $\cos \theta_{V}=0$. For higher values of $r_{V}$, the $\cos \chi$ dependence from the fifth term is seen at low $\cos \theta_{V}$, and $-\cos \chi$ from the sixth term is seen at high $\cos \theta_{V}$. The contributions from the first, second, and fourth terms are small, as is evident from the lack of a large $\sin ^{2} \theta_{V}$ component in any of the plots. 


\section{Appendix B}

\section{$p_{T}$ Discrepancy}

As mentioned previously (see Chapter 5) there is a significant discrepancy between the distributions of the component of the $D$ momentum transverse to the beam axis, in data and Monte Carlo. Figure B.1 clearly shows that $p_{T}$ in Monte Carlo events peaks lower than in data. We correct for the difference in the two distributions by using the ratio of the number of data events to Monte Carlo events in bins of $p_{T}$, as weights in the maximum likelihood fit. These weights are listed in Table B.1. As the result of weighting the Monte Carlo events, the value for $r_{V}$ returned by the fit is shifted to $2.08 \pm 0.11$ from $1.99 \pm 0.11$, while the value for $r_{2}$ remains unchanged.

As a result of weighting the Monte Carlo events, the total D momentum is affected in a significant way, as can be seen in Figure B.2. There is a poorer match between the $p_{T}$-weighted Monte Carlo events and data, than the unweighted ones. The mean of the D momentum distribution of data events is $59.5 \mathrm{GeV}$. For Monte Carlo events, the mean is $59.4 \mathrm{GeV}$ in the unweighted case, and $61.5 \mathrm{GeV}$ when the events are weighted by $p_{T}$ based weights. In fact, part of the difference seen in the result for $r_{V}$ could be due to the fact that the D momentum in Monte Carlo no longer matches data. To account for the discrepancy in the D momentum distribution, we calculate weights based on the ratio of data and $p_{T}$-weighted Monte Carlo events in bins of $\mathrm{D}$ momentum. These weights are listed in Table B.2. The results for the form factor ratios with this fit are $0.63 \pm 0.08$ for $r_{2}$, and $2.05 \pm 0.11$ for $r_{V}$. These numbers represent the effect due purely to the discrepancy between the $p_{T}$ distributions. The 


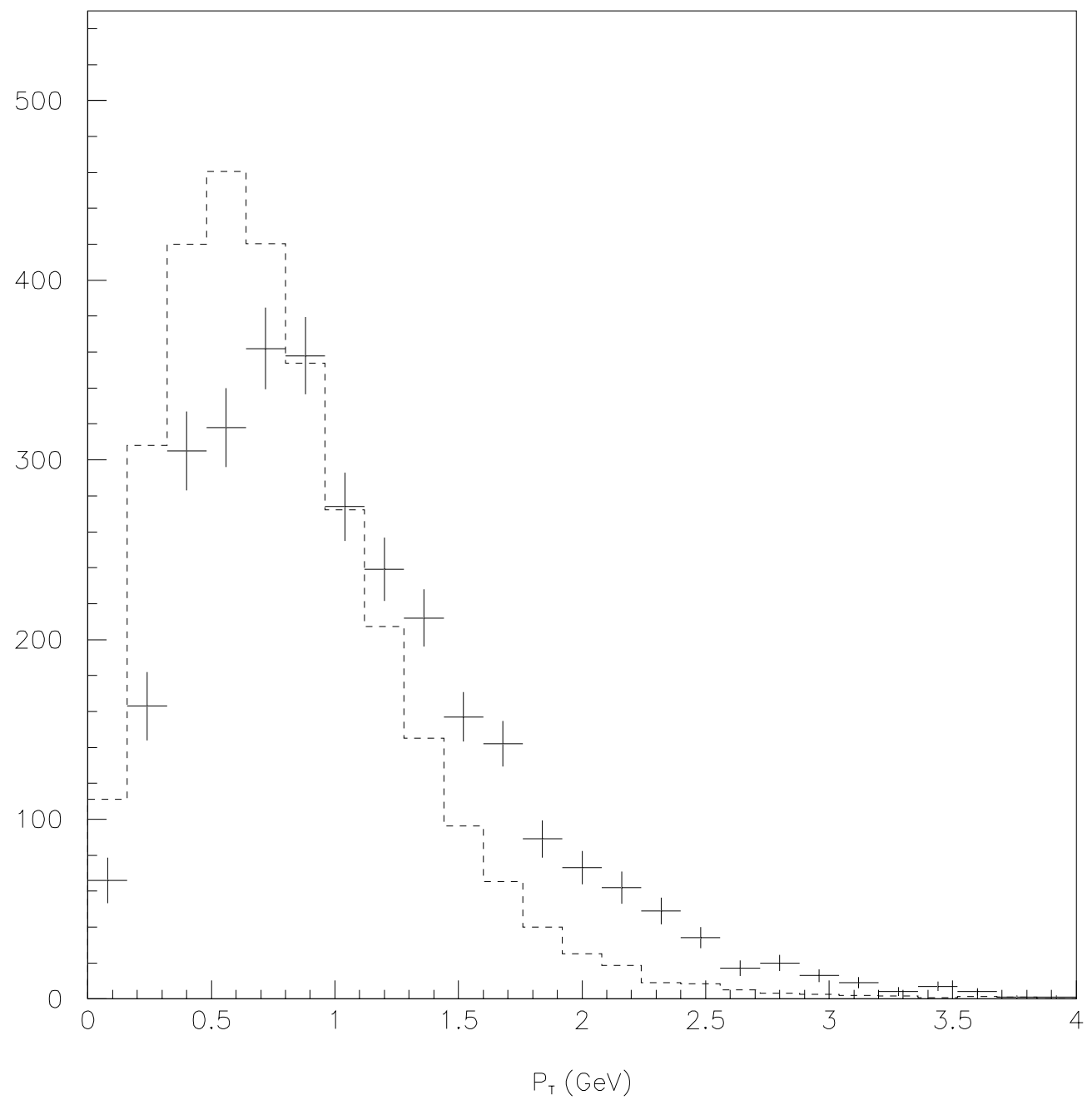

Figure B.1: Distribution of the component of D momentum transverse to the $z$-axis in data (cross hatches) and Monte Carlo (histogram). 


\begin{tabular}{|c|c|}
\hline$p_{T}$ Range $(\mathrm{GeV})$ & Weight \\
\hline $0.0-0.2$ & $0.60 \pm 0.09$ \\
$0.2-0.4$ & $0.58 \pm 0.05$ \\
$0.4-0.6$ & $0.69 \pm 0.04$ \\
$0.6-0.8$ & $0.85 \pm 0.05$ \\
$0.8-1.0$ & $1.01 \pm 0.06$ \\
$1.0-1.2$ & $1.07 \pm 0.07$ \\
$1.2-1.4$ & $1.34 \pm 0.09$ \\
$1.4-1.6$ & $1.63 \pm 0.13$ \\
$1.6-1.8$ & $2.31 \pm 0.19$ \\
$1.8-2.0$ & $2.22 \pm 0.27$ \\
$2.0-2.4$ & $3.93 \pm 0.38$ \\
$2.4-3.0$ & $4.67 \pm 0.57$ \\
$>3.0$ & $3.95 \pm 0.81$ \\
\hline
\end{tabular}

Table B.1: Weights used to correct for the discrepancy between $p_{T}$ distributions in data and Monte Carlo. The statistical uncertainty on each weight is due mostly to the error on the number of data events in each bin, and is not used to determine the form-factor ratios.

\begin{tabular}{|c|c|}
\hline Momentum Range $(\mathrm{GeV})$ & Weight \\
\hline $0-40$ & $1.11 \pm 0.05$ \\
$40-60$ & $1.02 \pm 0.04$ \\
$60-80$ & $0.88 \pm 0.04$ \\
$80-100$ & $1.09 \pm 0.06$ \\
$100-140$ & $0.87 \pm 0.07$ \\
$>140$ & $0.63 \pm 0.14$ \\
\hline
\end{tabular}

Table B.2: Weights calculated from the distribution of $\mathrm{D}$ momentum, where the Monte Carlo events have been weighted by $p_{T}$ based weights. 


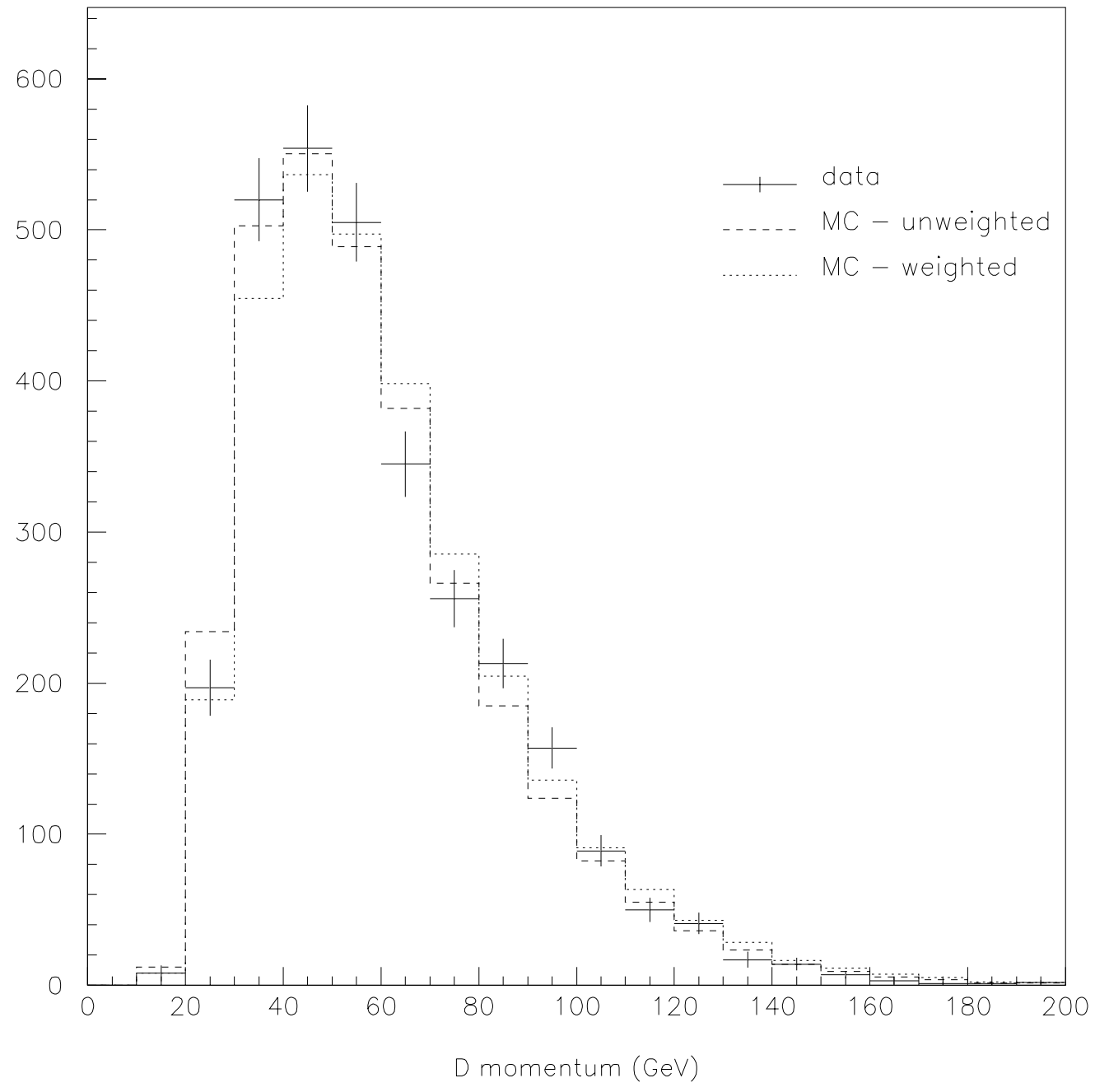

Figure B.2: D momentum distribution in data (cross-hatches) and Monte Carlo (histograms), where the Monte Carlo are plotted with and without $p_{T}$ based weights. 


\begin{tabular}{|l|c|c|}
\hline Fit Type & $r_{2}$ & $r_{V}$ \\
\hline Standard & $0.64 \pm 0.08$ & $1.99 \pm 0.11$ \\
$p_{T}$ weights & $0.64 \pm 0.08$ & $2.08 \pm 0.11$ \\
$p_{T}+$ D mom. weights & $0.63 \pm 0.08$ & $2.05 \pm 0.11$ \\
\hline
\end{tabular}

Table B.3: Results from three types of fits. The error is statistical. Systematic shift is not applied.

systematic shift for $r_{2}$ is -0.01 , and for $r_{V}$ it is +0.06 . In each case the shift is smaller than the systematic error (0.09 for $r_{2}$, and 0.08 for $\left.r_{V}\right)$. All the results are listed in Table B.3. 


\section{Bibliography}

[1] Gagnon, P., SCIPP-93/47, Ph.D. thesis, University of California, Santa Cruz, 1993.

[2] Richman, J.D., P.R. Burchat, Rev. Mod. Phys., Vol. 67, No. 4, 1995.

[3] Wise, M.B., CALT-68-1721, Proceedings of the Sixth Lake Louise Winter Institute, Chateau Lake Louise (17-23 February 1991), 222, and references therein, 1991.

[4] Review of Particle Physics, Phys. Rev. D 54, 1996.

[5] Isgur, N., M.B. Wise, Phys. Rev. D 42, 2388, 1990.

[6] Isgur, N., D. Scora, B. Grinstein, and M.B. Wise, Phys. Rev. D 39, 799, 1989.

[7] Scora, D., N. Isgur, Phys. Rev. D 52, 2783, 1995.

[8] Wirbel, M., B. Stech, and M. Bauer, Z. Phys. C 29,637, 1985.

[9] Altomari, T., and L. Wolfenstein, Phys. Rev. D 37, 681, 1988.

[10] Gilman, F.J., and R.L. Singleton, Phys. Rev. D 41, 93, 1990.

[11] Körner, J.G., and G. A. Schuler, Z. Phys. C 38, 511, 1988 [Erratum: C 41,690, 1989].

[12] Stech, B., Z. Phys. C 75,245, 1997.

[13] Simone, J.N., Nucl. Phys. Proc. Suppl. 47, 17, 1996. 
[14] Ball, P., V.M. Braun, and H.G. Dosch, Phys. Rev. D 44,3567, 1991.

[15] Anjos, J.C., et al., Phys. Rev. Lett. 65, 2630, 1990.

[16] Kodama, K., et al., Phys. Lett. B 274, 246, 1992.

[17] Frabetti, P.L., et al., Phys. Lett. B 307, 262, 1993.

[18] Bernard, C.W., Z.X. El-Khadra, and A. Soni, Phys. Rev. D 45, 869, 1991; Phys. Rev. D 47, 998, 1993.

[19] Lubicz, V., G. Martinelli, M.S. McCarthy, and C.T. Sachrajda, Phys. Lett. B 274, 415, 1992.

[20] Bhattacharya, T., and R.Gupta, Nucl. Phys. Proc. Suppl. 42, 935, 1995.

[21] Abada, A., et al., Nucl. Phys. B 416, 675, 1994.

[22] Allton, C.R., et al., Phys. Lett. B 345, 513, 1995.

[23] Nieves, M., et al., Report No. SHEP-94-95-09, presented at LATTICE 94, 12th International Symposium on Lattice Field Theory, Bielefeld, Germany, Sept.27Oct.1, 1994.

[24] Bowler, K.C., et al., U. of Edinburgh Report No. EDINBURGH-94-546, 1994.

[25] Gusken, S., et al., Nucl. Phys. Proc. Suppl 47, 485, 1996.

[26] Appel, J.A., Ann. Rev. Nucl. Part. Sci. 42 367, 1992, and references therein; D.J. Summers et al, Proceedings of the $X X V I I^{\text {th }}$ Rencontre de Moriond, Electroweak Interactions and Unified Theories, Les Arcs, France (15-22 March 1992) 417 ;

[27] Amato S.,et al, Nucl. Instrum. Methods A 324, 535, 1993.

[28] Fernow, R., "Introduction to Experimental Particle Physics", Cambridge University Press, 1986. 
[29] Damerell C.J.S., Report No. RAL-P-95-008, presented at 23rd Annual Summer Institute on Particle Physics, SLAC, Stanford, CA., July 10-21, 1995.

[30] Gagnon P., P. Burchat, and P. Casper, Offline Document 88, E791 internal documentation.

[31] Purohit M., Offline Document 248, E791 internal documentation.

[32] Brieman L., J.H. Friedman, R.A. Olshen, and C.J. Stone, "Classification and Regression Trees", Chapman and Hall, New York, 1984.

[33] Schmidt, D.M., R.J. Morrison, and M.S. Witherell, Nucl. Instr. Meth. A328,547, 1992.

[34] CERN Application Software Group, "MINUIT - Function Minimization and Error Analysis", Version 92.1, 1992.

[35] Bengtsson H.-U. and T. Sjostrand, Computer Physics Commun. 46 43, 1987.

[36] Sjostrand T. and M. Bengtsson, Computer Physics Commun. 43 367, 1987.

[37] Purohit M., Offline Document 125, E791 internal documentation.

[38] Mihalcea, D., Offline Document 334. E791 internal documentation.

[39] Aitala, E. M., et al., Phys. Rev. Lett. 80, 1393, 1998.

[40] Körner J. G., K. Schilcher, M. Wirbel, and Y. L. Wu, Z. Phys. C 48, 663, 1991.

[41] Ryd A., private communication with code originally written by R. Culbertson.

[42] Wolfram S., "Mathematica: a System for Doing Mathematics by Computer", Addison-Wesley Publishing Co., Redwood City, California, 1991. 